\title{
FIDSSA 2017 Congress Abstracts Oral Presentations
}

\section{ID: 8435}

Category: Infection Control (ICSSA)

Permission: Yes

Co-Author 1: Messina, A

Co-Author 1 Institution: Netcare Hospitals

Co-Author 2: Swart, K

Co-Author 2 Institution: Netcare Hospitals

Co-Author 3: Maslo, C

Co-Author 3 Institution: Netcare Hospitals

Co-Author 4: van den Bergh, D

Co-Author 4 Institution: Netcare Hospitals

E-mail Address: angeliki.messina@netcare.co.za

Country: South Africa

\section{ABSTRACT TITLE: IMPLEMENTATION OF A MOBILE APPLICATION FOR HAND HYGIENE MONITORING: SMART, SIMPLE AND INTERACTIVE}

\section{Introduction}

Hand Hygiene $(\mathrm{HH})$ is globally regarded as the single most effective infection prevention intervention used to reduce the spread of micro-organisms in healthcare settings. However, healthcare workers' compliance to $\mathrm{HH}$ is estimated at $<50 \%$. Comprehensive $\mathrm{HH}$ programmes are thought to include multi-modal strategies with focus on core elements such as: infrastructure, knowledge, awareness, measurement, feedback and behaviour change. This study aimed to implement a novel mechanism of $\mathrm{HH}$ monitoring across a network of 56 private South African hospitals.

\section{Methods}

A mobile application (app) was developed as a platform for data collection of $\mathrm{HH}$ observations according to the World Health Organisation's five moments campaign. An additional measurement of 'bare below the elbows (BBE)' was included. Access to the app was granted to identified observers following completion of a training module and competency assessment. Observations were required to be recorded weekly and a compliance dashboard was designed and distributed weekly to enable regular feedback to front-line staff.

\section{Results}

A total of 259283 observations for $\mathrm{HH}$ were recorded during the 47-week study period of which 214869 were compliant (82.9\%). Evaluation of $\mathrm{HH}$ compliance per moment indicates: before patient contact $81.7 \%(n=63486$ ); before aseptic task $91.8 \%$
( $n=17$ 284); after body fluid exposure risk 93.0\% ( $n=19$ 175); after patient contact $84.8 \%(n=55355)$ and; after contact with patient's surroundings $77.6 \%(n=59569)$. Average BBE compliance was $89.6 \%$ ( $n=131725$ ). A significant improvement in overall $\mathrm{HH}$ compliance was noted comparing data of 2016 to 2017 ( $p=<0.05$ ). Compliance of all categories of staff has improved over time 2016 vs 2017 . Improvements from doctors ( $72.3 \%$ vs $75.3 \%)$, registered nurses $(90.4 \%$ vs $91.5 \%)$ and pharmacy staff $(73.4 \%$ vs $79.2 \%)$ were significant $(p<0.05)$

\section{Conclusion}

$\mathrm{HH}$ compliance of healthcare workers in this study was markedly higher than that of previous studies. The implementation of the mobile application made data collection quick, simple and allowed for the availability of real time data to front-line staff to drive improvement.

\section{ID: 8680}

Category: Travel Medicine (SASTM)

Permission: Yes

Co-Author 1: Ntshoe, G

Co-Author 1 Institute: Division of Public Health Surveillance and Response, National Institute for Communicable Diseases

Co-Author 2: Tlagadi, A

Co-Author 2 Institute: South African Field Epidemiology Training Programme, National Institute for Communicable Diseases

Co-Author 3: Motladiile, T

Co-Author 3 Institute: Division of Public Health Surveillance and Response, National Institute for Communicable Diseases, North West Provincial Department of Health

Co-Author 4: McCarthy, K

Co-Author 4 Institute: Division of Public Health Surveillance and Response, National Institute for Communicable Diseases

Co-Author 5: Mokate, 0

Co-Author 5 Institute: North West Provincial Department of Health

Co-Author 6: Frean, J

Co-Author 6 Institute: Centre for Emerging Zoonotic and Parasitic Diseases, National Institute for Communicable Diseases

E-mail Address: genevien@nicd.ac.za

Country: South Africa 


\section{ABSTRACT TITLE: ODYSSEAN MALARIA OUTBREAK AT A BUSH LODGE IN MADIKWE GAME RESERVE, NORTH WEST PROVINCE, OCTOBER-NOVEMBER 2015}

\section{Introduction}

In November 2015, the National Institute for Communicable Diseases (NICD) was notified of a malaria case at a lodge in Madikwe Game Reserve, North West Province - usually a non-transmission area in South Africa. A few other people with fever, headache and 'flu-like symptoms at the same establishment were reported. An investigation to determine the possible cause/source of the illnesses was undertaken.

\section{Methods}

A structured questionnaire was used to gather information on demographic, clinical and exposure history. Interviews were conducted with employees and managers; blood samples were collected. Environmental assessment of the residence and immediate surroundings was conducted. Blood smear microscopy and PCR analysis for the detection of malaria parasites were done.

\section{Result}

Four laboratory-confirmed malaria cases were identified. All were female, employed, and resided at the lodge staff residences. Dates of symptom onset were 17 October 2015 (case 1), 21 October (case 2), 22 October (cases 3,4). Fever was reported in all; headache, dizziness and painful joints in three. Two were admitted to hospital. No recent blood transfusions were reported. None of the cases had travelled to malaria-endemic areas. However, travel history to possible malaria transmission areas was reported in eight of the 33 staff members interviewed. Cases 1 and 2 occupied adjoining rooms, cases 3 and 4 shared a room. Staff residences were within $50-60 \mathrm{~m}$ of the parking bay for establishment vehicles, including those returning from malaria-endemic areas. There was no evidence of free-standing water that could enable mosquito breeding. No asymptomatic infections were identified: all laboratory investigations for malaria were negative.

\section{Conclusion}

It is most likely that the cases acquired malaria from an imported, infected mosquito - the phenomenon of odyssean malaria. Spraying vehicles with a pyrethroid insecticide with residual effect would reduce the risk of transporting vector mosquitoes but this would be difficult to implement.

\section{ID: 8683}

Category: Antimicrobial Resistance (SAASP)

Permission: Yes

Co-Author 1: Wang, $Y$

Co-Author 1 Institute: Pharmacy, Frere Hospital; Faculty of Pharmacy, Rhodes University

Co-Author 2: Singh, $S$

Co-Author 2 Institute: Pharmacy, Frere Hospital; Faculty of Pharmacy, Rhodes University
Co-Author 3: Parrish, A

Co-Author 3 Institute: Department of Medicine, Frere Hospital; Department of Medicine, Cecilia Makiwane Hospital, Mdantsane

Co-Author 4: Stead, D

Co-Author 4 Institute: Division of Infectious Diseases, Department of Medicine, Frere Hospital; Department of Medicine, Cecilia Makiwane Hospital

E-mail Address: f.collett-white@doctors.org.uk

Country: South Africa

ABSTRACT TITLE: ASSESSING THE IMPACT OF ANTIMICROBIAL STEWARDSHIP WARD ROUNDS USING AN APP-BASED DATA COLLECTION TOOL IN TWO PUBLIC HOSPITALS IN THE EASTERN CAPE, SOUTH AFRICA

Frere Hospital is a 900-bed tertiary/speciality public hospital in the Eastern Cape, South Africa. The burden of disease consists of chronic conditions, acute and chronic infections with a major impact of HIV and TB.

The main challenges in our institution are a lack of resources (staff and finances) but also a lack of awareness of the importance of rational antimicrobial use. As a clinical pharmacist at Frere Hospital, I attend the Antimicrobial Stewardship rounds weekly with the ID Specialist and team which commenced in 2015 and we recently looked at an app as a tool to assess the impact of antimicrobial stewardship ward rounds. Attendance at the conference would be a fantastic opportunity to learn from other hospitals and pharmacists across Southern Africa and share our skills.

As mentioned above, one of the challenges in the institution is finances and we do not have a pharmacy budget for such.

\section{ID: 8358}

Category: Clinical Microbiology (SASCM)

Permission: Yes

Co-Author 1: Chomba, R

Co-Author 1 Institute: University of the Witswatersrand Co-Author 2: Moeng, $S$

Co-Author 2 Institute: University of the Witswatersrand

Co-Author 3: Lowman, W

Co-Author 3 Institute: University of the Witswatersrand

E-mail Address: irungurispah@yahoo.com

Country: South Africa

\section{ABSTRACT TITLE: PROCALCITONIN-GUIDED ANTIBIOTIC THERAPY FOR SUSPECTED AND CONFIRMED SEPSIS OF PATIENTS IN A SURGICAL-TRAUMA INTENSIVE CARE UNIT}

\section{Introduction}

Procalcitonin (PCT) is a useful sepsis marker to guide duration of antimicrobial therapy. PCT-guided algorithms have demonstrated value in reducing duration of antibiotic therapy in critical care patients. There is a paucity of data supporting the use of PCT-guided 
antibiotic algorithms in trauma patients and in patients from developing countries.

\section{Methods}

A prospective study was conducted in the surgical trauma intensive care unit (ICU) at Charlotte Maxeke Johannesburg Academic Hospital in April 2014 to July 2015 in a two period cross-over design. Patients with suspected or confirmed sepsis were recruited consecutively in two periods of almost equal length. In the first period, 40 patients were recruited as controls and antibiotics were discontinued as per standard of care. In the second period, 40 patients were recruited into the intervention group and antibiotics were discontinued if the PCT decreased by $\geq 80 \%$ from the peak PCT level, or to an absolute value of less than $0.5 \mu \mathrm{g} / \mathrm{L}$. Antibiotic duration was the primary outcome. Patients were followed up for 28 days from the first sepsis event.

\section{Results}

For the first sepsis event the intervention group had a mean antibiotic duration of 9.3 days while the control group had a mean duration of 10.9 days $(p=0.10)$. The mean duration of treatment was 12.0 days for a second episode of sepsis in the control group and 9.6 days in the intervention group $(p=0.09)$. Clinician compliance to the PCT algorithm was $62.5 \%$. The intervention group had more antibiotic-free days (7.8 days) compared to the control group ( 3.9 days) $(p=0.004)$. The length of ICU stay and length of hospital stay for the two groups were similar. The in-hospital mortality was reduced in the intervention group (15\%) compared to the control group (30\%).

\section{Conclusion}

Our data supports the use of PCT-guided algorithms for antibiotic stewardship in surgical trauma patients. Clinician compliance would likely increase the benefits observed in our study.

\section{ID: 8382}

Category: Clinical Microbiology (SASCM)

Permission: Yes

Co-Author 1: van Schalkwyk, E

Co-Author 1 Institute: Centre for Healthcare-Associated Infections, Antimicrobial Resistance and Mycoses, National Institute for Communicable Diseases

Co-Author 2: Shuping, L

Co-Author 2 Institute: Centre for Healthcare-Associated Infections, Antimicrobial Resistance and Mycoses, National Institute for Communicable Diseases

Co-Author 3: Ismail, $\mathrm{H}$

Co-Author 3 Institute: Centre for Healthcare-Associated Infections, Antimicrobial Resistance and Mycoses, National Institute for Communicable Diseases

Co-Author 4: Thomas, J

Co-Author 4 Institute: Centre for Healthcare-Associated Infections, Antimicrobial Resistance and Mycoses, National Institute for
Communicable Diseases

Co-Author 5: Govender, N

Co-Author 5 Institute: Centre for Healthcare-Associated Infections, Antimicrobial Resistance and Mycoses, National Institute for Communicable Diseases; Faculty of Health Sciences, University of the Witwatersrand

E-mail Address: erikab@nicd.ac.za

Country: South Africa

\section{ABSTRACT TITLE: INDEPENDENT RISK FACTORS ASSOCIATED WITH CANDIDA AURIS CANDIDAEMIA IN SOUTH AFRICA - AN ANALYSIS OF NATIONAL SURVEILLANCE DATA, 2016-2017}

\section{Introduction}

Candida auris is a globally-emerging, multi-drug-resistant invasive fungal pathogen. We aimed to determine risk factors for $C$. auris candidaemia to inform prevention and management.

\section{Methods}

Culture-confirmed cases of candidaemia were detected through active, national laboratory-based surveillance at all public- and private-sector hospitals, January 2016 - June 2017. We defined a case of candidaemia as any patient with Candida cultured from blood. Fungal isolates were identified using Bruker mass spectrometry or ITS region sequencing. We used multivariable logistic regression to assess clinical/demographic factors associated with C. auris candidaemia versus other Candida species from patients admitted to 25 enhanced surveillance sites.

\section{Results}

Among 2044 cases of candidaemia, 206 (10\%) were caused by C. auris, with a median age of 54years (IQR:36-64) versus 26years (IQR: $0-57)$ among non- $C$. auris cases. Most cases in both groups were male (C. auris: 60\% [122/203]; non-C. auris: 53\% [960/1806]). C. auris cases predominantly occurred in Gauteng Province (191/205, 93\%) and in private-sector hospitals (154/206, 75\%). Among 50 C. auris cases with clinical data, crude in-hospital mortality was 49\% (23/47), versus $42 \%$ (333/788) for non-C. auris cases $(p=0.368)$. Both groups had high proportions with invasive devices and intensive care unit admission. Twenty-nine per cent (12/41) and 85\% (35/41) of C. auris cases had received prior antifungal/ antibacterial therapy. Being admitted to a private-sector hospital increased the odds of C. auris candidaemia three-fold (aOR 3.6; $95 \% \mathrm{Cl} 1.62-7.77$ ). Other risk factors included older age (aOR 1.01 for every year; $95 \% \mathrm{Cl}$ : 1.01-1.03) and longer hospital stay before first positive blood culture (aOR 1.01 for every day admitted; $95 \% \mathrm{Cl}$ : 1.01-1.03).

\section{Conclusion}

Older patients with prolonged hospitalisation and admission to private-sector facilities had increased odds of $C$. auris candidaemia possibly owing to exposure to multiple invasive devices and prior antimicrobial therapy. 


\section{ID: 8483}

Category: Infection Control (ICSSA)

Permission: Yes

Co-Author 1: Duse, PA

Co-Author 1 Institute: University of the Witwatersrand

Co-Author 2: Conradie, DD

Co-Author 2 Institute: Council of Scientific and Industrial Research Co-Author 3: Mcloughlin, J

Co-Author 3 Institute: TB/HIV Care Association

Co-Author 4: Mbongo, LCM

Co-Author 4 Institute: South African Police Service

Co-Author 5: Moolman, A

Co-Author 5 Institute: National Health Laboratory Service; University of the Witwatersrand

Co-Author 6: Devenish, L

Co-Author 6 Institute: National Health Laboratory Service; University of the Witwatersrand

E-mail Address: lesley.devenish@gmail.com

Country: South Africa

\section{ABSTRACT TITLE: RISK ASSESSMENT FOR THE TRANSMISSION OF INFECTIOUS DISEASES IN THE SOUTH AFRICAN POLICE SERVICES (SAPS)}

\section{Introduction}

In 2012, the Constitutional Court made a landmark judgment against the Minister of Correctional Services where it was found that the Department of Correctional Services (DCS) failed to take reasonable steps to prevent transmission of tuberculosis (TB). Infection prevention and control (IPC) is a collective term for activities, policies and procedures designed to prevent and control the transmission of infectious diseases within various environments (e.g. healthcare centres, detention and correctional centres, other institutions and the community).

The continuum of an offender's journey starts at the crime scene and arrest, transportation between the police station (SAPS) holding cells and the courts until admission to a correctional centre. Infection transmission risks occur at each stage of the continuum and stringent IPC measures are critical to prevent infection spread.

\section{Methods}

An IPC audit tool was developed for SAPS. Five assessments were done. The assessment included process mapping, a questionnaire, observations, interviews and measurement of ventilation in SAPS cell blocks. Common IPC non-conformances were identified.

\section{Results}

- Ventilation issues were identified - variation between $25 \mathrm{~L} / \mathrm{s} /$ per person to $50 \mathrm{~L} / \mathrm{s} /$ per person which is insufficient for a medium-risk population.

- Aggregation and overcrowding are significant risks for TB transmission.
- Improvement opportunities included open window enforcement, medical screening, separation protocols, hand hygiene, environmental hygiene, medication management and occupational health and safety.

- IPC procurement under the current supply chain system is problematic.

\section{Conclusion}

The risk assessment highlighted the need for the implementation of an IPC programme in the SAPS. A Provincial SAPS Committeeapproved training programme was commenced in June 2017.

\section{ID: 8344}

Category: Infectious Diseases (IDSSA)

Permission: Yes

Co-Author 1: Ahmad, W

Co-Author 1 Institute: University of Veterinary and Animal Sciences, Subcampus-Jhang

Co-Author 2: Song, $Y$

Co-Author 2 Institute: Key Laboratory of Zoonosis Research, Ministry of Education, Institute of Zoonosis, College of Veterinary Medicine, Jilin University

Co-Author 3: Gong, Y

Co-Author 3 Institute: Key Laboratory of Zoonosis Research, Ministry of Education, Institute of Zoonosis, College of Veterinary Medicine, Jilin University

Co-Author 4: Wang, $X$

Co-Author 4 Institute: Key Laboratory of Zoonosis Research, Ministry of Education, Institute of Zoonosis, College of Veterinary Medicine, Jilin University

Co-Author 5: Gao, J

Co-Author 5 Institute: Key Laboratory of Zoonosis Research, Ministry of Education, Institute of Zoonosis, College of Veterinary Medicine, Jilin University

Co-Author 6: Duan, M

Co-Author 6 Institute: Key Laboratory of Zoonosis Research, Ministry of Education, Institute of Zoonosis, College of Veterinary Medicine, Jilin University

Co-Author 7: Guan, Z

Co-Author 7 Institute: Key Laboratory of Zoonosis Research, Ministry of Education, Institute of Zoonosis, College of Veterinary Medicine, Jilin University

Co-Author 8: Maolin, Z

Co-Author 8 Institute: Key Laboratory of Zoonosis Research, Ministry of Education, Institute of Zoonosis, College of Veterinary Medicine, Jilin University

Co-Author 9: Khan, I

Co-Author 9 Institute: University of Veterinary and Animal Sciences, Subcampus-Jhang

Email Address: waqas.hussain@uvas.edu.pk

Country: Pakistan \& China 


\section{ABSTRACT TITLE: RABIES VIRUS DOWN-REGULATES THE EXPRESSION OF ACTIN AND MICROTUBULE-ASSOCIATED PROTEINS IN NEURONS}

\section{Background}

Rabies virus (RABV) causes neuronal dysfunction, and alters the structural morphology of dendritic spines by inducing changes in cytoskeleton which is further comprised of actin, microtubule and intermediate filaments. For microtubule, EB3 is a microtubule plusend binding protein that stabilises microfilaments and belongs to the third family member of RP/EB group. The p140cap is an interacting partner of EB3 that, together with $\mathrm{EB} 3$, plays a vital role in postsynaptic density, dendritic spine functions and morphology.

\section{Methods}

The gene expression and respective protein contents of EB3 and p140cap were compared in neuronal cells under the effect of fixed and street strain of RABV. Furthermore, gene expression levels of important actin binding, microtubule and synapse-related proteins were also studied. In this study, immunofluorescence, western blot and real time PCR were carried out to compare the gene expression and corresponding protein contents of different cytoskeleton related proteins.

\section{Results}

Both strains of RABV significantly reduced the gene expression and protein contents of essential cytoskeleton-related proteins like EB3 and p140cap. However, the street strain considerably inhibited the transcription level of p140cap, but had no significant effect on its protein level. The fixed strain produced fractured microtubules in fixed neurons, and down-regulated different microtubule and actinassociated proteins, while up-regulated the level of Tesk2 gene. In neuronal cells, the fluorescence localisation of EB3 protein was random and varied at 48 hour and 98 hours of post-infection.

\section{Conclusions}

Rabies virus possibly alters the neuronal structures by changing the gene expression levels of integral protein binding partners of microtubules and actin. Most importantly, EB3 and p140cap are vital in maintaining the morphology of dendritic spines. These results could help us to understand the intricate relationship between RABV and microfilaments.

\section{ID: 8695}

Category: Infectious Diseases (IDSSA)

\section{Permission: Yes}

Co-Author 1: Tau, N

Co-Author 1 Institute: Centre for Enteric Diseases, National Institute for Communicable Diseases, a division of the National Health Laboratory Service

Co-Author 2: Smith, A

Co-Author 2 Institute: Centre for Enteric Diseases, National Institute for Communicable Diseases, a division of the National Health Laboratory Service
Co-Author 3: Keddy, K

Co-Author 3 Institute: Centre for Enteric Diseases, National Institute for Communicable Diseases, a division of the National Health Laboratory Service

E-mail Address: nomsat@nicd.ac.za

Country: South Africa

\section{ABSTRACT TITLE: MOLECULAR CHARACTERISATION OF SALMONELLA TYPHI ISOLATES FROM SOUTH AFRICA, 2012-2014}

\section{Introduction}

Typhoid fever is a grave systemic infection caused by the bacterium Salmonella enterica serovar Typhi (Salmonella Typhi). It has been estimated that Salmonella Typhi causes approximately 720 illnesses per 100000 populations annually, in Africa. Of more concern is the emergence and spread of the Salmonella Typhi H58 haplotype from Asia. Salmonella Typhi $\mathrm{H} 58$ is highly clonal and has been associated with multi-drug resistance to first-line antimicrobials and reduced susceptibility to fluoroquinolones, the current treatment regimen for patients with typhoid fever. The aim of this study was to use molecular techniques for the characterisation of Salmonella Typhi isolates from patients in South Africa, 2012-2014.

\section{Methods}

For the period 2012-2014, 195 Salmonella Typhi isolates were selected from the culture collection at the Centre for Enteric Diseases (CED). These isolates were subjected to conventional PCR in order to screen for the presence of the Salmonella Typhi H58. Isolates were also analysed using a multiple-locus variable-number tandem-repeats analysis (MLVA) assay that targeted five highly polymorphic variable-number tandem-repeats loci. The assay included PCR amplification of fluorescently labelled VNTR loci and analysis of amplicons by capillary electrophoresis.

\section{Results}

Fifty-four percent of isolates (105/195) were identified as Salmonella Typhi H58, while $46 \%$ (95/195) were identified as non-H58 Salmonella Typhi strains. MLVA analysis of the 195 isolates revealed 155 MLVA subtypes; the most common subtypes were: STyMT-121, STyMT-114, STyMT-102, STyMT-130, STyMT-132, STyMT-136 and STyMT-139. The MLVA subtyping tool successfully discriminated among Salmonella Typhi $\mathrm{H} 58$ isolates and identified MLVA types that were exclusively associated with Salmonella Typhi H58.

\section{Conclusion}

The dissemination of the Salmonella Typhi H58 haplotype within South Africa is concerning. Molecular subtyping tools, such as MLVA, can be very useful in disease tracking and for the investigations of typhoid fever outbreaks caused by the Salmonella Typhi H58.

\section{ID: 8539}

Category: Sexually Transmitted Diseases (STDSSA)

Permission: Yes 
Co-Author 1: Happel, A

Co-Author 1 Institute: University of Cape Town

Co-Author 2: Zauchenberger, $C$

Co-Author 2 Institute: University of Cape Town

Co-Author 3: Gamielden, H

Co-Author 3 Institute: University of Cape Town

Co-Author 4: Jaumdally, SZJ

Co-Author 4 Institute: University of Cape Town

Co-Author 5: Dabee, $S$

Co-Author 5 Institute: University of Cape Town

Co-Author 6: Barnabas, S

Co-Author 6 Institute: University of Cape Town

Co-Author 7: Bekker, L

Co-Author $\mathbf{7}$ Institute: Desmond Tutu HIV Foundation

Co-Author 8: Jaspan, $\mathrm{H}$

Co-Author 8 Institute: University of Cape Town

Co-Author 9: Froissart, $R$

Co-Author 9 Institute: University of Montpellier

Co-Author 10: Passmore, J

Co-Author 10 Institute: University of Cape Town

Email Address: HPPANN001@myuct.ac.za

Country: South Africa

\section{ABSTRACT TITLE: COMPARING COMMERCIAL PROBIOTIC AND VAGINAL LACTOBACILLUS STRAINS TO EVALUATE WHETHER PROBIOTICS MARKETED FOR VAGINAL HEALTH IN SOUTH AFRICA ARE SUITABLE FOR BACTERIAL VAGINOSIS TREATMENT}

\section{Introduction}

Bacterial vaginosis (BV) is prevalent globally and associated with increased risk of sexually transmitted infections, including HIV. Antibiotic treatment of BV has high recurrence rates ( $50 \%$ within 6 months). Adjunctive treatment with probiotics improves efficacy and durability of treatment. We compared characteristics of Lactobacillus strains from commercially-available vaginal probiotics to those isolated from vaginal samples.

\section{Methods}

Lactobacillus spp. were isolated from commercial vaginal probiotics (10 isolates from 5 products), and from cervico-vaginal fluid (60 isolates from healthy South African women). Species were identified using a MALDI-TOF biotyper. Bacterial size, growth kinetics, effect on $\mathrm{pH}$ and on BV associated vaginal species (clinical and ATCC strains of Gardnerella vaginalis and Prevotella bivia) and antibiotic profiles were measured.

\section{Results}

Lactobacillus spp. isolated from healthy women were L. crispatus, L. jensenii, L. gasseri, L. mucosae and L. vaginalis which were absent from all probiotic products (which contained L. reuteri, L. rhamnosus and L. acidophilus). Clinical isolates grew better at lower $\mathrm{pH}$ (ph 2 - ph 6.0) than probiotic strains, and lowered pH more effectively (3.6 vs. 4.4). Culture supernatants from both probiotic and clinical strains promoted on ATCC G. vaginalis-growth, while inhibiting clinical $G$. vaginalis and all P. bivia strains. All strains were resistant to metronidazole, the most common $\mathrm{BV}$ treatment, while the majority of probiotic (8/10) and clinical strains (58/60) were sensitive to clindamycin. All isolates were sensitive to rifamycins (used in TB treatment) and other common antibiotics (including penicillin and amoxicillin). However, probiotic strains were more sensitive to rifamycins and less sensitive to penicillin and amoxicillin than clinical isolates.

\section{Conclusion}

Characteristics of commercial probiotic Lactobacillus strains are highly variable and did not contain the predominant species isolated from the healthy female genital tract. Clinical L. crispatus isolates had comparable characteristics, and a better probiotic profile than commercial probiotic strains.

\section{ID: 8520}

Category: Clinical Microbiology (SASCM)

Permission: Yes

Co-Author 1: Reddy, $\mathrm{K}$

Co-Author 1 Institute: Division of Microbiology, NHLS Tygerberg and Stellenbosch University

Co-Author 2: Whitelaw, A

Co-Author 2 Institute: Division of Microbiology, NHLS Tygerberg and Stellenbosch University

E-mail Address: kessendri.reddy@nhls.ac.za

Country: South Africa

\section{ABSTRACT TITLE: CAN WE TRUST THE XPERTS? AN EVALUATION OF THE XPERT MRSA/SA BC SYSTEM AND ASSESSMENT OF POTENTIAL CLINICAL IMPACT}

\section{Introduction}

Positive blood cultures with Gram-positive cocci in clusters may result in inappropriate antibiotic administration or delayed administration of appropriate therapy until identification is confirmed. The Xpert MRSA/SA BC system allows rapid differentiation between methicillin-sensitive $S$. aureus (MSSA), methicillin-resistant S. aureus (MRSA) and coagulase-negative staphylococci (CoNS) in positive blood cultures. This prospective study evaluated the assay's performance and potential clinical benefit in an academic laboratory in Cape Town.

\section{Methods}

The Xpert assay was compared to culture-based methods on blood cultures with Gram-positive cocci in clusters. Discrepancies were resolved using Vitek 2 and $16 S$ PCR if necessary. Potential impact of implementation on antibiotic use was assessed by clinical review, 
and classified as de-escalation (stopping or changing to a narrowerspectrum agent) or administration of a more appropriate agent (if current therapy was inactive against the organism).

\section{Results}

Of 231 patents, 16 had MRSA, 41 had MSSA and 174 had CoNS based on phenotypic methods. Clinical information was obtained for 184 patients. The Xpert system showed a sensitivity and specificity of $100 \%$ (95\% Cl $93.7-100 \%$ and $97.8-100 \%$ respectively) for S. aureus identification. The sensitivity for detecting methicillin resistance in S. aureus was $93.8 \%$ (95\% Cl 71.7-98.9\%); specificity was 100\% (95\% Cl 91.4-100\%) after resolution of a single discrepancy. The error/ invalid rate was $2.58 \%$. Potential impact included a reduction in time-to-final result by $30.5 \mathrm{~h}$, with more timely de-escalation in 29 patients and administration of an appropriate antistaphylococcal agent in 17 patients.

\section{Conclusion}

The Xpert MRSA/SA BC assay performs well in differentiating MRSA, MSSA and CoNS in positive blood cultures. It has potential to improve antibiotic utilisation in approximately $25 \%$ of patients. These benefits must be balanced against the assay's cost in resource-constrained settings. A formal clinical trial may provide further evidence to determine the benefit of the assay.

\section{ID: 8548}

Category: Clinical Microbiology (SASCM)

Permission: Yes

Co-Author 1: Lutchminarain, $\mathrm{K}$

Co-Author 1 Institute: National Health Laboratory Service; University of KwaZulu-Natal

Co-Author 2: Mlisana, K

Co-Author 2 Institute: National Health Laboratory Service; University of KwaZulu-Natal

Co-Author 3: Kajee, A

Co-Author 3 Institute: National Health Laboratory Service

E-mail Address: Lutchminarain@ukzn.ac.za

Country: South Africa

\section{ABSTRACT TITLE: INTRODUCTION OF DRUG RESISTANT TB - REFLEX TESTING IN KWA-ZULU NATAL - A DESCRIPTIVE ANALYSIS OF MTBDRSL RESISTANT ISOLATES OVER A 9 MONTH PERIOD}

\section{Introduction}

Following WHO recommendations for both the shortened multidrug resistant (MDR) treatment regimen and use of the GenoType MTBDRsI Line Probe Assay (SL-LPA), the South African Department of Health together with NHLS developed a "DR-TB reflex" testing algorithm. The aim of this is to identify those MDR- or rifampicinresistant TB patients who can be commenced on the shortened MDR-TB treatment regimen.

\section{Methods}

874 GenoType MTBDRsl assays performed directly on sputum samples as part of DRTB reflex testing over a four-month period were analysed. The DR-TB reflex testing set comprises a panel of tests: Auramine-O, MGIT960 culture, LPA-first line, SL-LPA and where necessary phenotypic drug susceptibility testing.

\section{Results}

Of the 874 samples processed, 109 (12.5\%)showed resistance to either flouroquinolones, SL injectable drugs (SLID) or both. Fluoroquinolone-resistant isolates showed mutations in the gyrA gene, with an absent gyrA WT2 and a corresponding positive gyrA mutation1 (MUT1) as the commonest pattern. The second commonest was an absent WT2 with corresponding MUT3C. The least common pattern was an absent WT3 with a corresponding MUT3C. Of note, eight isolates had mutation bands with no corresponding absent WT (6 MUT3C, 1 MUT3B, 1 MUT1). No resistance was detected in the gyrB gene.

For the SLID, > 95\% of the isolates showed mutation in the rrsgene. The commonest pattern showed an absent WT1 and a corresponding MUT1. The second commonest was an absent WT2 with a corresponding WT2. An absent WT1 with no corresponding mutation was observed in six isolates. Resistance in the eis gene was noted in only two isolates, one having an absent WT1 with corresponding MUT1, the other, an absent WT1 with no corresponding mutation.

\section{Conclusion}

Preliminary data show $12.5 \%$ prevalence of drug resistant TB in the tested period. GyrA and rrs gene mutations are the commonest mutations.

\section{ID: 8707}

Category: Antimicrobial Resistance (SAASP)

Permission: Yes

Co-Author 1: Farley, E

Co-Author 1 Institute: University of Cape Town

Co-Author 2: Stewart, A

Co-Author 2 Institute: University of Cape Town

Co-Author 3: Boyles, $T$

Co-Author 3 Institute: University of Cape Town

Co-Author 4: Davies, M

Co-Author 4 Institute: University of Cape Town

E-mail Address: elisefarley@gmail.com

Country: South Africa

ABSTRACT TITLE: KNOWLEDGE, ATTITUDES AND

PERCEPTIONS OF ANTIMICROBIAL RESISTANCE AMONGST PRIVATE PRACTICE PATIENTS AND PRESCRIBERS IN SOUTH AFRICA 


\section{Introduction}

Antimicrobial resistance (AMR) may be the next global health crisis. We aimed to describe South African patients' and prescribers' AMR knowledge, attitudes and perceptions (KAP).

\section{Methods}

We conducted a cross-sectional KAP survey among a convenience sample of prescribers and patients in South Africa. We used logistic regression to examine associations between knowledge and antibiotic use, beliefs, or behaviours.

\section{Results}

Mean patient $(n=403)$ knowledge scores (out of 14) were higher in females $(p=0.0005)$ and those with more education $(p=0.015)$; $76 \%$ believed the human body, rather than the infectious agent, becomes resistant to antibiotics. After adjusting for education and sex, a 1-unit increase in patient knowledge was associated with increased odds of the following beliefs: (i) important to finish the antibiotic course (aOR 1.34; 95\% Cl:1.16, 1.66); (ii) over-use impacts AMR (aOR 1.3; 95\% Cl:1.18, 1.43), but reduced odds of (iii) demanding antibiotics should be given (aOR 0.84; 95\% Cl:0.74,0.94); (iv) feeling relieved (aOR $0.89 ; 95 \% \mathrm{Cl}: 0.81,0.97$ ) or happy (aOR $0.905 ; 95 \% \mathrm{Cl}: 0.82,0.99)$ when prescribed antibiotics.

Prescribers $(n=175)>55$ years old had lower median knowledge scores than younger prescribers $(p=0.0005)$. Those who infrequently prescribe antibiotics when unnecessary had higher knowledge scores $(p=0.01) ; 70 \%$ of prescribers feel pressure from patients to prescribe antibiotics.

Prescribers with higher knowledge scores were more likely to believe the following: (i) to decrease AMR, narrow spectrum antibiotics should be used (aOR 1.41; 95\% Cl:1.03, 1.92), (ii) explaining to patients disease features which should prompt follow-up (aOR 1.76; $95 \% \mathrm{Cl}: 1.01$, 30.74). Prescribers with higher knowledge scores were less likely to report that antibiotics cannot harm the patient if not needed and less likely to prescribe when not necessary (aOR 0.55 $95 \% \mathrm{Cl}: 0.33,0.91)$.

\section{Conclusion}

The association between knowledge and behaviour/perceptions suggests that increasing patient and prescriber knowledge could influence antibiotic use behaviours.

\section{ID: 8542}

Category: Sexually Transmitted Diseases (STDSSA)

Permission: Yes

Co-Author 1: Mitchev, N

Co-Author 1 Institute: University of KwaZulu-Natal

Co-Author 2: Singh, R

Co-Author 2 Institute: University of KwaZulu-Natal, National Health Laboratory Service

Co-Author 3: Khanyile, $\mathrm{T}$
Co-Author 3 Institute: University of KwaZulu-Natal

Co-Author 4: Naidoo, J

Co-Author 4 Institute: Centre for the AIDS Programme of Research in South Africa (CAPRISA)

Co-Author 5: Garrett, N

Co-Author 5 Institute: Centre for the AIDS Programme of Research in South Africa (CAPRISA)

Co-Author 6: Mlisana, KP

Co-Author 6 Institute: School of Nursing and Public Health, Discipline of Public Health Medicine, University of KwaZulu-Natal; School of Laboratory Medicine and Medical Sciences, University of KwaZulu Natal, National Health Laboratory Service, Centre for the AIDS Programme of Research in South Africa (CAPRISA)

E-mail Address: nireshninaidoo@live.co.za

Country: South Africa

\section{ABSTRACT TITLE: COMPARISON OF NUCLEIC ACID AMPLIFICATION TESTS TO DETECT SEXUALLY TRANSMITTED INFECTIONS FROM VAGINAL SPECIMENS}

\section{Introduction}

The use of nucleic acid amplification tests (NAATs) is the recommended diagnostic method in the management and control of STIs. Our study compared the performance of five commercially available NAATs, to determine which would be most suitable for use in our setting. To optimise the potential impact on women's health management we concurrently screened for bacterial vaginosis (BV) and vulvovaginal candidiasis (VVC).

\section{Methods}

Vaginal swab (ESwab, COPAN, Italy) specimens were collected from 251 young women attending an urban clinic in KwaZulu-Natal to screen for STIs and bacterial vaginosis (BV) using six new technology NAAT assays (Anyplex ${ }^{\mathrm{TM}}$ II STI-7 Detection, BD MAX CT/GC/TV, Fasttrack STD9, Xpert ${ }^{\oplus} \mathrm{CT} / \mathrm{NG}$, Thermofisher probes and $\mathrm{BD}^{\mathrm{MAX}}{ }^{\mathrm{T}}$ Vaginal Panel). All assays were processed as per manufacturers' instructions.

\section{Results}

We determineda prevalence of 5\% Neisseria gonorrhoeae (NG), 14\% Chlamydia trachomatis (CT), 4\% Trichomonas vaginalis (TV), 5\% Mycoplasma genitalium (MG), 34\% Mycoplasma hominis (MH), 19\% Ureaplasma urealyticum (UU) and 51\% Ureaplasma parvum (UP). The sensitivity, specificity, PPV and NPV of all assays was comparable (> 90\%). Overall, $80 \%$ of the women were positive for at least one or a combination of STI-causing organisms. The prevalence of welldefined STIs (NG, CT, TV and MG) was $27 \%$. Of all the cases with NG infection ( $n=12), 42 \%$ were co-infected with $C T$, and $15 \%$ of cases with $\mathrm{CT}$ also had NG.The BD MAX ${ }^{\mathrm{TM}}$ Vaginal Panel determined the prevalence of BV and VVC to be $49 \%$ and $42 \%$ respectively.

\section{Conclusion}

All STI NAAT assays performed well, each with its own advantages over the others. Head-to-head comparisons of these new STI assays are rare in our setting and assist service providers with choosing the best option for their clinical management needs. 


\section{ID: 8439}

Category: Antimicrobial Resistance (SAASP)

Permission: Yes

Co-Author 1: Lowings, $M$

Co-Author 1 Institute: University of Pretoria

Co-Author 2: Kock, M

Co-Author 2 Institute: University of Pretoria, Pretoria, Gauteng, South Africa/Tshwane Academic Division, National Health Laboratory Service

Co-Author 3: Coetzee, J

Co-Author 3 Institute: Ampath Laboratories

Co-Author 4: Hoosien, E

Co-Author 4 Institute: Ampath Laboratories

Co-Author 5: van Greune, J

Co-Author 5 Institute: Ampath Laboratories

Co-Author 6: Peirano, G

Co-Author 6 Institute: University of Calgary

Co-Author 7: Ehlers, M

Co-Author 7 Institute: University of Pretoria, Tshwane Academic Division, National Health Laboratory Service

Co-Author 8: Chen, L

Co-Author 8 Institute: Rutgers University

Co-Author 9: Kreiswirth, B

Co-Author 9 Institute: Rutgers University

Co-Author 10: Pitout, J

Co-Author 10 Institute: University of Calgary

E-mail Address: lowingsmichelle@gmail.com

Country: South Africa

\section{ABSTRACT TITLE: KLEBSIELLA PNEUMONIAE ST307: A SILENT PATHOGEN ON THE RISE IN SOUTH AFRICA}

\section{Introduction}

There is a desperate need for molecular surveillance systems, especially in developing countries, with the ability to recognise and track the emergence of high-risk antimicrobial resistant (AMR) clones in a real-time fashion. Whole genomic sequencing (WGS) and rapid PCR identification were used to track the emergence of a novel OXA-181-producing K. pneumoniae clone ST307 in South Africa.

\section{Methods}

Illumina WGS was performed on a collection (2011 to 2016) of clinical $K$. pneumoniae with blaOXA-48-like carbapenemases from South Africa and used this information to design PCR primers for the identification of ST307 and its association with blaOXA-181 on IncX3 plasmids.

\section{Results}

Whole genomic sequencing on 28 isolates showed that K. pneumoniae ST307 belonged to two clades. Clade I was associated with blaCTX-M-15 and corresponded to ST307 sequences deposited in Genbank $(n=9)$. A novel ST307 clade II (that differs from clade I in approximately 100 SNPs) contained blaOXA-181 and was associated with IS 3000 on IncX3 plasmids. Subsequent PCR screening of K. pneumoniae with blaOXA-48-like showed that ST307 clade II with blaOXA-181 was first detected during 2012 in Johannesburg. In 2013 and 2014, 20/135 (15\%) of K. pneumoniae with blaOXA48-like tested positive for ST307 clade II with blaOXA-181 and was found in Alberton, Johannesburg and Pretoria. During 2015 and 2016 the numbers of ST307 clade II increased exponentially; $297 / 499(60 \%)$ of $K$. pneumoniae with blaOXA-48-like belonged to ST307. This clade had subsequently spread to 10 other cities (i.e. Bloemfontein, Emalahleni, Ermelo, East London, Klerksdorp, Krugersdorp, Mbombela, Polokwane, Secunda, Vanderbijlpark) across six different provinces (i.e. Eastern Cape, Gauteng, Free State, Limpopo, Mpumalanga, North West).

\section{Conclusion}

This study described the rapid emergence over a six-year period of K. pneumoniae ST307 clade II with blaOXA-181 in South Africa and highlighted the importance of using WGS to develop molecular surveillance methods for tracking emerging AMR clones in a rapid fashion. Such methods will aid with the implementation of measures to curb the spread of antibiotic resistance.

\section{ID: 8538}

Category: Antimicrobial Resistance (SAASP)

Permission: Yes

Co-Author 1: Ismail, N

Co-Author 1 Institute: Department of Medical Microbiology, Faculty of Health Sciences, University of Pretoria

Co-Author 2: Peters, RP

Co-Author 2 Institute: Department of Medical Microbiology, Faculty of Health Sciences, University of Pretoria; Department of Medical Microbiology, CAPHRI School of Public Health and Primary Care, Maastricht University Medical Centre, Maastricht University

Co-Author 3: Vally, SO

Co-Author 3 Institute: Centre for Tuberculosis, WHO Supranational TB Reference Laboratory, National Institute for Communicable Diseases, National Health Laboratory Service

Co-Author 4: Ismail, NA

Co-Author 4 Institute: Department of Medical Microbiology, Faculty of Health Sciences, University of Pretoria; Centre for Tuberculosis, WHO Supranational TB Reference Laboratory, National Institute for Communicable Diseases, National Health Laboratory Service

E-mail Address: NABILAI@NICD.AC.ZA

Country: South Africa \& The Netherlands

ABSTRACT TITLE: EVALUATION OF METHODS FOR GENERATION OF IN VITRO MUTANTS RESISTANT TO BEDAQUILINE, CLOFAZIMINE AND LINEZOLID FROM MYCOBACTERIUM TUBERCULOSIS REFERENCE STRAINS 


\section{Introduction/Aim}

The introduction of bedaquiline and repurposing of linezolid and clofazimine for the management of drug-resistant TB has resulted in improved cure rates. However, there is a paucity of information regarding Mycobacterium tuberculosis resistance acquisition mechanisms. We sought to determine the optimum methodology for resistance induction to these drugs and their resistanceassociated variants (RAVs) using Whole Genome Sequencing (WGS).

\section{Methods}

ATCC reference strains with unique resistance profiles were used to compare two in vitro resistance generation approaches; serial passaging through increasing antimicrobial pressure (five strains) and spontaneous generation (twostrains). This was done for bedaquiline, clofazimine and linezolid. Generated mutants were confirmed with MGIT 960 drug susceptibility testing (DST). WGS and bioinformatic analysis were performed to identify induced RAVs, which were then classified as known or novel variants. In addition, clofazimine-induced resistant mutants were subjected to bedaquiline MGIT 960 DST to assess cross resistance.

\section{Results}

Mutants were successfully generated for both approaches in all strains investigated. Generated mutants for each drug using both approaches showed up to an eight-fold increase compared to currently proposed critical concentration values. Spontaneously generated mutants showed a broader range of MIC values (from 2-8 $\mu \mathrm{g} / \mathrm{ml}$ ) for each drug and were obtained within a month from initiation. Mutants generated through serial passaging exhibited high MIC values $(>8 \mu \mathrm{g} / \mathrm{ml}$ ) and these were obtained after at least five passages (> 3 months). RAVs occurred in the rplC (linezolid), rv0678 (bedaquiline and clofazimine) and/or atpE (bedaquiline) regions. We identified both known and novel mutations for bedaquiline and clofazimine within respective genetic targets. Additionally, clofazimine resistant mutants (from both approaches) were cross resistant to bedaquiline without any previous drug exposure.

\section{Discussion/Conclusion}

Both approaches were capable of mutant generation from various ATCC strains with the spontaneous generation approach being preferred as it is rapid. Mutations identified correspond to known mutations, particularly for linezolid. In addition, for bedaquiline and clofazimine, novel mutations were identified, which warrant further study.

\section{ID: 8687}

Category: Antimicrobial Resistance (SAASP)

Permission: Yes

Co-Author 1: Wang, $Y$

Co-Author 1 Institute: Frere Hospital, Rhodes University

Co-Author 2: Collet-White, F
Co-Author 2 Institute: Thames Valley and Wessex Leadership Academy

Co-Author 3: Singh, $S$

Co-Author 3 Institute: Frere Hospital, Rhodes University

Co-Author 4: Stead, D

Co-Author 4 Institute: Frere Hospital, Walter Sisulu University

E-mail Address:Trisha_Wang@yahoo.com

Country: South Africa \& United Kingdom

\section{ABSTRACT TITLE: ANTIBIOTIC CONSUMPTION AFTER TWO YEARS OF ANTIMICROBIAL STEWARDSHIP WARD ROUNDS IN THE MEDICINE DEPARTMENT AT A TERTIARY HOSPITAL IN THE EASTERN CAPE}

\section{Introduction}

Rising antimicrobial resistance is challenging health practitioners in East London as it is across the country and globally. Reducing antimicrobial consumption rates can potentially reverse this trend. Weekly antimicrobial stewardship (AMS) ward rounds have been implemented in the Internal Medicine Department at Frere Hospital since 2015. We sought to assess if this intervention had impacted antibiotic consumption.

\section{Methods}

The RxSolution ${ }^{\circledast}$ pharmacy reporting tool was modified to facilitate the extraction of antibiotic consumption data (ward stock and pharmacy dispensed). This was performed for the five medical wards at Frere for April 2014 - March 2015 (pre- AMS rounds), and April 2016 - March 2017 (post - AMS implementation). Trimethoprimsulfamethoxazole and anti-tuberculosis drugs were excluded. The consumption units were converted manually to World Health Organisation defined daily doses (DDD) per 1000 in-patient days. Bed occupancy data for the relevant periods was obtained from the information technology department. Data analysis was done using Microsoft Excel.

\section{Results}

All the following antibiotic consumption value are presented in DDD per 1000 in-patient days. The overall antibiotic consumption of the Internal Medicine Department decreased from 830 in $2014 / 15$ to 682 in 2016/17 (18\% reduction). Broad-spectrum intravenous antibiotics targeting community-acquired infections, demonstrated a reduction of ceftriaxone usage from 229 to 148 (35\% reduction), and an increase in amoxicillin-clavulanic acid from 0.05 to 2.89. Antibiotics used for hospital acquired and resistant infections, revealed an increased use of carbapenems (5.68 to 11.8) and piperacillin-tazobactam (0.54 to 0.95$)$.

\section{Conclusion}

The implementation of weekly antimicrobial stewardship rounds has coincided with an overall reduction in antibiotic consumption, and notably in the use of ceftriaxone. The increased use of carbapenems and piperacillin-tazobactam likely reflect an increased awareness of hospital-acquired infections among prescribers. A further study can be done looking at the antibiotic consumption pattern changes in relation to local antibiotic resistance rates. 


\section{ID: 8506}

Category: Clinical Microbiology (SASCM)

Permission: Yes

Co-Author 1: Nyazema, KB

Co-Author 1 Institute: University of Pretoria

Co-Author 2: Malinga, LA

Co-Author 2 Institute: Tuberculosis Research Platform Unit, Medical Research Council

Co-Author 3: Antiabong, JF

Co-Author 3 Institute: Center for Infectious Disease and Experimental Therapeutics, Baylor Institute

Co-Author 4: Mbelle, NM

Co-Author 4 Institute: Department of Medical Microbiology, University of Pretoria

E-mail Address: nyazemak@gmail.com

Country: South Africa \& United States of America

\section{ABSTRACT TITLE: INVESTIGATION OF DISCREPANCIES BETWEEN PHENOTYPIC AND MOLECULAR METHODS FOR DETECTION RIFAMPICIN SUSCEPTIBILITIES FROM A TERTIARY DIAGNOSTIC LABORATORY IN PRETORIA: TOWARDS CONSENSUS IN RPOB MUTATIONS}

\section{Introduction}

Multi-drug resistant tuberculosis (MDR-TB) caused by resistance to at least rifampicin (RIF) and isoniazid (INH) remains a major health concern worldwide. To curb the spread of MDR-TB, GenoType ${ }^{\oplus}$ MTBDRplus was endorsed for the accurate detection and treatment initiation of TB cases whilst waiting for phenotypic drug susceptibility test (DST) results. Discrepancies between phenotypic DST and GenoType ${ }^{\circledR}$ MTBDRplus have been observed. It is thus important to correctly characterise different rpoB mutations causing different RIF susceptibilities. We investigated discrepancies between GenoType ${ }^{\oplus}$ MTBDRplus and phenotypic DST for RIF and sequenced the rpoB gene in susceptible and resistant isolates.

\section{Methods}

Forty isolates provided by the NHLS-TB Laboratory were characterised. The isolates were cultured and tested for drug susceptibility, phenotypically using BACTEC ${ }^{\mathrm{TM} M G \mathrm{~T}^{\mathrm{TM}}}$ (RIF critical concentration $=1 \mu \mathrm{g} / \mathrm{ml}$ ) and genotypically with GenoType ${ }^{\oplus}$ MTBDRplus according to manufacturer's instructions. A PCR amplification of the $r p o B$ gene was performed and the amplicons were sequenced. The sequences of susceptible isolates were used to develop the consensus sequence in silico and used for comparison with the resistant strains to query results obtained with the H37Rv reference strain.

\section{Results}

There was $100 \%$ agreement $(\mathrm{kappa}=1.0$ ) between phenotypic drug susceptibility testing and Sanger sequencing. A relatively lower agreement of $90 \%$ (kappa $=0.8$ ) was observed between phenotypic DST and GenoType ${ }^{\oplus}$ MTBDRplus assay. Five mutations were detected and occurred at different frequencies with S531L (50\%) occurring most often followed by D516V (20\%), H526S (10\%), H526D (10\%) and a TTC insertion at position $1297(10 \%)$. The consensus sequence constructed gave similar results to $\mathrm{H} 37 \mathrm{Rv}$ reference strain.

\section{Conclusion}

Discrepancies occur because molecular tests detect resistance at a molecular level and not the ultimate phenotypic expression. Sequencing could help minimise phenotypic and genotypic discrepancies found in most diagnostic laboratories. Similar results between H37Rv and the developed consensus sequence highlight better performance of Sanger sequencing for DST in comparison to GenoType ${ }^{\oplus}$ MTBDRplus.

\section{ID: 8507}

Category: Infection Control (ICSSA)

Permission: Yes

Co-Author 1: Sallie, S

Co-Author 1 Institute: Netcare Milpark Hospital

Co-Author 2: Mukupa, E

Co-Author 2 Institute: Netcare Milpark Hospital

Co-Author 3: Georgieva, $\mathrm{H}$

Co-Author 3 Institute: Netcare Milpark Hospital

E-mail Address: shakiera.sallie@netcare.co.za

Country: South Africa

\section{ABSTRACT TITLE: \#CAUTI MUST FALL}

\section{Introduction}

In 2013, the Catheter Associated Urinary Tract Infection (CAUTI) rate in an internationally renowned 346-bed private hospital was of concern at 3.62 per 1000 catheter days. Of the 346 beds, 109 are specialised ICU and High Care beds and many of the patients are catheterised. Urinary catheterisation of patients is required for close output monitoring of critically ill patients, selective surgery and urethral obstruction. CAUTI is the most common healthcareassociated infection. CAUTIs result in prolonged hospitalisation, increased use of antimicrobials, with the potential to develop multidrug resistant organisms and increased hospital and patient costs.

\section{Methods}

In 2013 a number of CAUTIs were present shortly after admission. A Standard Operating Procedure (SOP) was drafted for'on admission' urinalysis on all patients. The SOP was rolled out to all units, training was given and CAUTI audits conducted. A CAUTI Champion was appointed in late 2013 and a multidisciplinary CAUTI committee was formed, which included an Urologist, Infection Control, Nursing Management and Unit Managers, and regular meetings were held. In addition, CAUTI Unit representatives were identified and trained. A CAUTI Workshop presented by the Urologist was arranged and CAUTI rounds were conducted in all units and gaps identified were 
discussed with possible solutions. A campaign for the early removal of "awake patients" catheters was developed and driven by IPC and unit CAUTI champions.

\section{Results}

The 2014 CAUTI rate/ 1000 catheter days was 2.81 down from 3.62 in 2013. In 2015 the CAUTI rate fluctuated to 2.85 and required further focus. By 2016, the CAUTI rate per 1000 catheter days decreased to 2.04 .

\section{Conclusion}

Collaborative team efforts, constant training, reminders to do admission urine tests, and audits is required to prevent CAUTIs and sustain a reduction. The nature of the patients at the hospital is such that many will require urine catheters, but management and prompt removal is of paramount importance.

\section{ID: 8355}

Category: Infectious Diseases (IDSSA)

Permission: Yes

Co-Author 1: Meiring, $S$

Co-Author 1 Institute: National Institute for Communicable Diseases

Co-Author 2: de Gouveia, L

Co-Author 2 Institute: National Institute for Communicable Diseases

Co-Author 3: Kleynhans, J

Co-Author 3 Institute: National Institute for Communicable Diseases

Co-Author 4: du Plessis, M

Co-Author 4 Institute: National Institute for Communicable Diseases

Co-Author 5: Ganesh, K

Co-Author 5 Institute: National Institute for Communicable Diseases

Co-Author 6: Quan, V

Co-Author 6 Institute: National Institute for Communicable Diseases

Co-Author 7: von Gottberg, A

Co-Author 7 Institute: National Institute for Communicable Diseases

Co-Author 8: Cohen, $\mathrm{C}$

Co-Author 8 Institute: National Institute for Communicable Diseases

E-mail Address: susan.meiring@nhls.ac.za

Country: South Africa

\section{ABSTRACT TITLE: MENINGOCOCCAL CARRIAGE AMONGST FIRST-YEAR STUDENTS ON ENTERING UNIVERSITY: IS THERE A NEED IN SOUTH AFRICA FOR MENINGOCOCCAL PREVENTION STRATEGIES?}

\section{Introduction}

Although peak incidence of invasive meningococcal disease (IMD) is in infants, a second peak is seen in adolescents due to their social interactions and high carriage of Neisseria meningitidis(NM). Adolescent carriage prevalence ranges from $3 \%$ in the African meningitis belt to $23 \%$ in the United Kingdom. IMD is a severe disease associated with outbreaks. Prevention strategies should be sought even with low IMD incidence.

\section{Methods}

To determine the NM carriage prevalence and carriage risk factors amongst first-year students upon entering university, we collected oropharyngeal swabs and questionnaires from students during registration week at two large universities in Western Cape (WC) and Gauteng Provinces (GA) in 2017. Swabs were placed directly into Todd Hewitt broth and incubated for 24 hours; plated onto Thayer Martin media and incubated for a further $24-48 \mathrm{hrs}$ in 5\% CO 2. NM colonies were confirmed using MALDI-TOF mass spectrometry. Risk factors for carriage were analysed using STATA version 14.0.

\section{Results}

Two-thousand-one-hundred-and-twenty-one students completed the questionnaire and swab, 901 (42\%) in WC and 1220 (58\%) in GA. Median age was 18 years, 41\% (876) were male and 0.8\% (16/1 985 with known HIV status) HIV-infected.

Sixty-six students (3\%, 95\% confidence interval (Cl) 2.5-3.9\%) were NM carriers, of which $52 \%(34 / 66, p=0.09)$ were male and all were HIV-uninfected.

When adjusting for home province (KwaZulu-Natal (KZN) (Odds Ratio (OR)3.3, Cl1.5-7, $\mathrm{p}=0.003$ ) and WC (OR3.4, Cl1.8-6.2, $\mathrm{p}=0.004$ ) compared to $\mathrm{GA}$ ), smoking (OR3.1, Cl1.9-5, $\mathrm{p}<0.001$ ), smoke exposure (OR4.7, Cl1.3-6.7, $\mathrm{p}<0.001$ ), intimate kissing partners (OR3.8, Cl2.2-6.5, $\mathrm{p}<0.001$ ), party (OR2.1, Cl1.3-3.4, $\mathrm{p}=0.003)$, nightclub (OR3.7, Cl2.6-6.1, $\mathrm{p}<0.001)$ or pub (OR3, Cl1.8$4.9, p<0.001)$ attendance in the last two weeks - home province (Eastern Cape adjusted - OR3.1, Cl1.1-8.8, $\mathrm{p}=0.004$; KZ aOR3.7, Cl1.7-8.3, $p=0.001$; WC aOR2.6, Cl1.4-5.0, $p=0.004$, compared to $\mathrm{GA})$, exposure to smoke (aOR2.9, $\mathrm{Cl} 1.3-6.7, \mathrm{p}=0.01$ ) and intimate kissing partners (aOR2.7, $\mathrm{Cl} 1.5-4.8, \mathrm{p}=0.001$ ) remained significant risk factors for carriage.

\section{Conclusion}

NM carriage in adolescents entering university was $3 \%$. Significant NM carriage risk factors were home province, secondary smoke exposure and intimate kissing. Targeting adolescent smoke exposure is a practical health promotion activity that could prevent meningococcal circulation.

\section{ID: 8372}

Category: Infectious Diseases (IDSSA)

Permission: Yes

Co-Author 1: Preiser, W

Co-Author 1 Institute: Division of Medical Virology, Faculty of Medicine \& Health Sciences, University of Stellenbosch, National Health Laboratory öService (NHLS) Tygerberg

Co-Author 2: Cronjé, $\mathrm{N}$

Co-Author 2 Institute: Division of Medical Virology, Faculty of Medicine \& Health Sciences, University of Stellenbosch

Co-Author 3: Ithete, NL 
Co-Author 3 Institute: Division of Medical Virology, Faculty of Medicine \& Health Sciences, University of Stellenbosch

Co-Author 4: Suliman, $T$

Co-Author 4 Institute: Division of Medical Virology, Faculty of Medicine \& Health Sciences, University of Stellenbosch

E-mail Address: preiser@sun.ac.za

Country: South Africa

\section{ABSTRACT TITLE: CORONAVIRUS DIVERSITY IN SOUTH AFRICAN BATS - A RISK FOR HUMAN HEALTH?}

\section{Introduction}

Emerging infectious diseases pose a major threat to human health. Bats are recognised as reservoir hosts for many important viruses with zoonotic potential, including coronaviruses (CoV). Following the emergence of SARS in 2002/03 and the subsequent identification of Rhinolophus sinicus as the likely ancestral SARS-CoV source, a wide diversity of bat CoV has been described worldwide. Our research, in transdisciplinary collaborations with ecologists and zoologists, aims to better understand CoV diversity and ecology in South African bats.

\section{Methods}

We conducted general surveillance and species-specific studies of bats across South Africa. Samples - including faecal pellets, saliva and urine swabs, and from voucher specimens sacrificed for museum collections, also blood and organs - were screened for CoV genome by a widely used Pan-CoV PCR assay and 816bp RGU fragment sequences (Drexler et al., 2010) used to construct ML trees in MEGA v7.

\section{Results}

An improved screening method greatly increased the CoV detection rate: 92 of 686 samples from 9 bat species were screening-positive: 66 for $a-C o V, 19$ for $\beta-C o V$, and 7 for both. Most sequences are a-CoVs, with $\sim 20 \%$ prevalence in Neoromicia capensis. Preliminary analyses of partial RdRp, nucleocapsid and spike gene fragments of novel $\beta-\mathrm{CoV}$ identified in Neoromicia and Pipistrellus bats show their close relatedness to BtCoV PML-PHE1/RSA/2011 (NeoCoV), previously found by us in a N. capensis and belonging to the same viral species as the recently emerged MERS-CoV, responsible for the ongoing outbreak in the Arabian Peninsula.

\section{Conclusions}

Extensive sampling allowed detection of $\alpha$ - and $\beta-\mathrm{CoV}$ from a wide range of bat species across large parts of South Africa. An improved screening approach yielded significantly more positives. There is substantial CoV diversity in southern African bats. Most importantly, our finding of additional MERS-CoV-related CoV will help address the unresolved question of the origin of this zoonotic pathogen.

\section{ID: 8644}

Category: Infectious Diseases (IDSSA)

Permission: Yes
Author: Enoch, A

Author Institute: National Health Laboratory Service, Groote Schuur Hospital

E-mail Address: annabelenoch@gmail.com

Country: South Africa

\section{ABSTRACT TITLE: HEPATITIS A SEROPREVALENCE IN SOUTH AFRICA. ARE WE IN EPIDEMIOLOGICAL TRANSITION?}

\section{Introduction}

Hepatitis A virus (HAV) is the most common cause of viral hepatitis worldwide. Infection with HAV is vaccine preventable, however, a vaccine against $H A V$ is not included in the Expanded Programme on Immunisation in South Africa (SA). South Africa was considered to be highly endemic for hepatitis $A$ in the past, hence there was no need for routine immunisation against the virus. Our hypothesis is that $S A$ is changing from a high to intermediate endemic setting for hepatitis A. To test our hypothesis, we conducted a cross-sectional seroprevalence pilot study in the one to seven-year age group in the Western Cape Province.

\section{Methods}

The samples for our study were specimens sent for routine diagnostics to referral hospitals in the Western Cape Province. We tested remaining serum of 482 samples sent for routine tests (of which only 481 were analysed), for hepatitis A total antibodies using a Siemens enzyme immunoassay. We also analysed hepatitis A test results from the National Health Laboratory Services (NHLS) Disa*Lab database at Groote Schuur hospital from 2009-2014, as well as hepatitis A surveillance data from the National Institute for Communicable Diseases (NICD) from 2009-2014, to look at the past hepatitis A prevalence trend.

\section{Results}

Our cross-sectional study showed the seroprevalence to be $44.1 \%$ in the one to seven-year age group. The NHLS data showed a seroprevalence of $<90 \%$ up to age 10 years, indicating intermediate endemicity. The NICD data showed an increased incidence of hepatitis $\mathrm{A}$ infection in the 7-40 year age group, suggesting an increasing proportion of a susceptible population to HAV infection.

\section{Conclusion}

Taken together, these results indicate the need for considerations in the development of vaccination policies against HAV infection in South Africa.

\section{ID: 8697}

Category: Infectious Diseases (IDSSA)

Permission: Yes

Co-Author 1: Dube, FS

Co-Author 1 Institute: Department of Molecular and Cell Biology, Faculty of Science, University of Cape Town, South Africa and Division of Medical Microbiology, Faculty of Health Science, University of Cape Town 
Co-Author 2: Kwambana-Adams, B

Co-Author 2 Institute: Vaccines and Immunity Theme, Medical Research Council Unit, The Gambia

Co-Author 3: Senghore, M

Co-Author 3 Institute: Vaccines and Immunity Theme, Medical Research Council Unit, The Gambia

Co-Author 4: Peter, NS

Co-Author 4 Institute: Vaccines and Immunity Theme, Medical Research Council Unit, The Gambia

Co-Author 5: Archibald, W

Co-Author 5 Institute: Vaccines and Immunity Theme, Medical Research Council Unit, The Gambia

Co-Author 6: Antonio, M

Co-Author 6 Institute: Vaccines and Immunity Theme, Medical Research Council Unit, The Gambia

E-mail Address: fdube82@gmail.com

Country: South Africa \& Gambia

\section{ABSTRACT TITLE: MOLECULAR EPIDEMIOLOGY OF PAEDIATRIC BACTERIAL MENINGITIS AND UTILITY OF GENOMICS IN ROUTINE INFECTIOUS DISEASE INVESTIGATION IN LIMITED-RESOURCE SETTING}

\section{Introduction}

Acute bacterial meningitis is most commonly caused by Neisseria meningitidis, Streptococcus pneumoniae (the "pneumococcus) and Haemophilus influenzae Type b. Although $N$. meningitidis is the leading cause of meningitis in West Africa, pneumococcal meningitis outbreaks are increasing. Microbiological culture methods are adversely affected by prior antibiotic use and reduced sensitivity. We report the molecular epidemiology of bacterial meningitis and utility of genomics data in infectious disease investigations in Senegal, a limited-resource setting.

\section{Methods}

Cerebrospinal fluid (CSF) specimens were collected from patients with suspected meningitis in Senegal from 2009 through 2016. CSF specimens were subjected to Gram staining, culture and rapid antigen testing. Quantitative PCR was performed to identify pneumococcus, meningococcus and $H$. influenzae spp. Latex agglutination and molecular serotyping were performed on samples. Antibiogram and whole genome sequencing were performed on pneumococcal isolates. Whole-genome re-sequencing (WGS) was performed on a subset of 53 pneumococcal isolates.

\section{Results}

Of the 1012 children enrolled (median age $=12$ months, $\mathrm{IQR}=2-31$ months) with suspected meningitis, at least one bacterial species was isolated from 33\% (116/351) of the CSF Pneumococci, meningococcus and $H$. influenzae spp accounted for $80 \%, 28 \%$ and $16 \%$ respectively. Of the 38 pneumococcal isolates subjected to WGS, most common vaccine serotypes were $1,5,14,6 \mathrm{~A}$ and $6 \mathrm{~B}(26 \%, 13 \%, 8 \%$ and $8 \%$ respectively). Non-vaccine serotypes were rare and included serotypes 24 (8\%) and 35D (3\%). Most pneumococcal isolates tested were susceptible to a broad range of antibiotics. A majority of serotypes were resistant to both trimethoprim-sulfamethoxazole and tetracycline. Further genomic analyses to uncouple these observations are in progress.

\section{Conclusion}

The occurrence of a pneumococcal serotype 1 and 5 meningitis outbreak three years after the introduction of PCV13 is alarming and calls for strengthening of meningitis surveillance and a reevaluation of the current vaccination programme in high risk countries.

\section{ID: 8688}

Category: Paediatric Infectious Diseases (SASPID)

Permission: Yes

Co-Author 1: Anderson, $\mathrm{K}$

Co-Author 1 Institute: University of Cape Town

Co-Author 2: Muloiwa, R

Co-Author 2 Institute: University of Cape Town

Co-Author 3: Davies, M

Co-Author 3 Institute: University of Cape Town

E-mail Address: kimanderson@law.co.za

Country: South Africa

ABSTRACT TITLE: TREATMENT OUTCOMES IN PERINATALLY-HIV-INFECTED CHILDREN AND ADOLESCENTS AFTER 10 YEARS ON ANTIRETROVIRAL THERAPY

\section{Introduction}

The burden of paediatric HIV in South Africa has shifted to older children and adolescents. Nevertheless, information on long-term treatment outcomes of perinatally-HIV(PHIV)-infected children is limited. We examined long-term immunologic and virologic outcomes of children who remained in care for $\geq 10$ years after starting antiretroviral therapy (ART).

\section{Methods}

We included PHIV-infected children who initiated ART at a Cape Town clinic between 2002 and 2005, and had follow-up for $\geq 10$ years from ART initiation date. CD4 counts and viral loads (VL) were analysed for each successive year on ART.

\section{Results}

The median follow-up among 127 patients included was 12.2 years (interquartile range [IQR] 11.1-13.0). At ART initiation, median age was 2.6 years (IQR 1.3-4.9) and mean CD4 percentage was 13.7\% (95\% Confidence Interval [CI] 13.6-13.9).The first ART regimen was non-nucleoside reverse transcriptase inhibitor (NNRTI)-based $(64 \%)$ or protease inhibitor (PI)-based (36\%). After 10 years since ART initiation, $49.6 \%$ of patients were on 1 st-line, $43.3 \%$ on 2 ndline, $3.1 \%$ on lamivudine monotherapy and $3.9 \%$ on no ART. After 10 years on combined ART, $87.5 \%$ had CD4 $>500$ cells $/ \mu$ l $(98 \%$ of those on 1st-line vs $79 \%$ of those on 2nd-line ART; $p=0.003)$. In those who had VL performed after 10 years, 74.3\% (81/109) had VL $<400$ copies $/ \mathrm{ml}(81.5 \%$ of those on 1 st-line vs $69.2 \%$ on 2 nd-line 
ART; $p=0.16)$. The 10-year probability of experiencing viral failure was $56.7 \%$ (95\% Cl 48.3-65.5), and was higher in children on NNRTIbased regimens vs PI-based regimens ( $65.8 \%$ vs $43.1 \%$; logrank test, $\mathrm{p}=0.04$ ). The 10 -year probability of switching to 2 nd-line ART was $45.7 \%$ (95\% Cl $37.5-54.8)$.

\section{Conclusion}

Virologic and immunologic outcomes are good overall in children who remain in care for $\geq 10$ years, but $>40 \%$ of children were on 2nd-line ART with poorer immunologic outcomes.

\section{ID: 8307}

\author{
Category: Sexually Transmitted Diseases (STDSSA) \\ Permission: Yes \\ Co-Author 1: Kenyon, C \\ Co-Author 1 Institute: ITM \\ Co-Author 2: Schwartz, I \\ Co-Author 2 Institute: ITM \\ Co-Author 3: Buyze, J \\ Co-Author 3 Institute: ITM \\ E-mail Address: chriskenyon0@gmail.com \\ Country: South Africa
}

\section{ABSTRACT TITLE: STRONG ASSOCIATION BETWEEN HIGHER RISK SEX AND HIV PREVALENCE AT REGIONAL LEVEL: AN ECOLOGICAL STUDY OF 27 SUB-SAHARAN AFRICAN COUNTRIES}

\section{Introduction}

It is unclear why HIV prevalence varies by nearly two orders of magnitude between regions within countries in sub-Saharan Africa. In this ecological study, we assess if HIV prevalence by region is associated with any of four markers of higher risk sexual behaviour: lifetime number of partners, multiple partners in past year, higher risk sex (defined as sex with non-cohabiting, non-marital partners) and age at debut.

\section{Methods}

We performed Pearson's correlation between the four behavioural risk factors and HIV prevalence by region in 47 nationally representative surveys from 27 sub-Saharan African countries, separately by gender. In addition, principal components analysis was used to reduce the eight risk factors (four for each gender) to two principal components (PCs). Mixed effects linear regression was used to assess the relationship between the resulting two PCs and HIV prevalence after controlling for the prevalence of male circumcision.

\section{Results}

HIV prevalence varied by a median 3.9-fold (IQR 2.9-8.5) between regions within countries. HIV prevalence was strongly associated with higher risk sex and, to a lesser extent, the other risk factors evaluated. Both PCs were strongly associated with HIV prevalence when assessed via linear regression.

\section{Conclusion}

Differences in sexual behaviour may underpin the large differences in HIV-prevalence between subpopulation within sub-Saharan African countries.

\section{ID: 8677}

Category: Infectious Diseases (IDSSA)

Permission: Yes

Co-Author 1: Gaelejwe, L

Co-Author 1 Institute: University of Witwatersrand

Co-Author 2: Prabdial-Sing, DN

Co-Author 2 Institute: University of Witwatersrand

Co-Author 3: Puren, PA

Co-Author 3 Institute: University of Witwatersrand

E-mail Address: lucy.gaelejwe@gmail.com

Country: South Africa

\section{ABSTRACT TITLE: PRELIMINARY EVALUATION OF DRIED BLOOD SPOTS TO TEST FOR HEPATITIS C VIRUS (HCV) ANTIBODIES AMONG PEOPLE WHO INJECT DRUGS, SEX WORKERS AND MEN WHO HAVE SEX WITH MEN}

Hepatitis $C$ virus infection is a significant cause of morbidity and mortality worldwide (Mahd Hanafiah et al., 2013). It has been estimated that 130-170 million people are chronically infected with HCV and 3-4 million new infections per year (Bennette $S$ et al., 2012). The highest prevalence of HCV infection was reported in Asia and Africa (Mohd Hanafiah et al., 2013). The prevalence of 2.1\%-2.8\% has been reported in sub-Saharan Africa (Lyden et al., 2014) and 0.1\%-1.7\% sero-prevalence reported in South Africa (Abuelhassan, 2012). However, HCV infection still remains as the most under-diagnosed virus leaving those populations that are infected unaware of their infection status (Tuaillan $E$ et al., 2009) especially in South Africa. It has been reported that the most-atrisk population are unaware of their $\mathrm{HCV}$ infection status including the high-risk groups (Bennett et al., 2012). This could be due to the stigma and discrimination they are faced with when accessing healthcare facilities and especially when they test HCV positive.

In order to diagnose HCV infection, patients have to undergo several phlebotomy procedures for multiple tests. This can pose a challenge for PWIDs, who are commonly referred to as 'hard sticks' and may be restricted for testing altogether (Dokubo EK et al., 2014). In some international settings, blood draws are not culturally accepted; as a result the testing of $\mathrm{HCV}$ is not a priority. Testing protocols may also limit HCV diagnosis; in other circumstances venipuncture is not a convenient method that is readily available and in other parts of the world diagnostic technology is limited therefore making the diagnosis of HCV in resource-limited settings a challenge (Dokubo EK et al., 2014).

There have been numerous HCV diagnostic tests which have been invented throughout the years, most of which are used in 
centralised laboratory facilities. These sample matrixes are cheaper, reliable and faster tests. These tests are easy to handle, store and can be transported from distant clinics to the centralised laboratory without refrigeration and retain the integrity of the specimen (Vinikoor et al., 2015). Various studies have validated the use of these sample matrixes such as the Dried Blood Spot (DBS) and found a sensitivity of $93.8-100 \%$ and a specificity of $94.0-100 \%$ (Bennett et al., 2012).

DBS offers less invasive measure of sample collection and they also have a potential to facilitate the detection of HCV antibodies (Dokubo EK et al., 2014). This could potentially lead to early diagnosis, reduced risk behaviours and better treatment effects (Dokubo EK et al., 2014). This will scale up testing for HCV infection among high-risk groups in South Africa, more especially in PWIDs group which has been reported to be the population with the highest HCV prevalence (Dokubo EK et al., 2014). It can also be expected that HCV prevalence data in South Africa among the most-at-risk population will be generated also have a positive improvement to the current South African healthcare outcomes.

This study is conducted at NICD and is part of a larger collaboration on hepatitis $C$ infection in key populations [people who inject drugs (PWID), sex workers (SWs) and men who have sex with men (MSM)] accessing HIV testing services in Cape Town, Durban, Pietermaritzburg, Mthatha, Port Elizabeth, Johannesburg and Pretoria. Currently, I do not have any funding for attending the conference. It would be of great pleasure if I could attend the conference because my study (research) offers new insights about sample matrixes for HCV testing to the larger population (research scientist clinicians and the general population) and hopefully will improve the current patient care treatment and HCV database for $\mathrm{HCV}$ in South Africa.

\section{ID: 8440}

Category: Paediatric Infectious Diseases (SASPID)

Permission: Yes

Co-Author 1: McMorrow, M

Co-Author 1 Institute: CDC-South Africa

Co-Author 2: Tempia, S

Co-Author 2 Institute: CDC-South Africa

Co-Author 3: Walaza, $S$

Co-Author 3 Institute: National Institute for Communicable Diseases (NICD)

Co-Author 4: Moyes, J

Co-Author 4 Institute: National Institute for Communicable Diseases (NICD)

Co-Author 5: Treurnicht, F

Co-Author 5 Institute: National Institute for Communicable Diseases (NICD)

Co-Author 6: Venter, M

Co-Author 6 Institute: University of Pretoria
Co-Author 7: Pretorius, M

Co-Author 7 Institute: Novartis

Co-Author 8: Hellferscee, 0

Co-Author 8 Institute: National Institute for Communicable Diseases (NICD)

Co-Author 9: Wolter, $\mathrm{N}$

Co-Author 9 Institute: National Institute for Communicable Diseases (NICD)

Co-Author 10: von Gottberg, A

Co-Author 10 Institute: National Institute for Communicable Diseases (NICD)

Co-Author 11: Nguweneza, A

Co-Author 11 Institute: National Institute for Communicable Diseases (NICD)

Co-Author 12: McAnerney, J

Co-Author 12 Institute: National Institute for Communicable Diseases (NICD)

Co-Author 13: Naby, F

Co-Author 13 Institute: University of KwaZulu-Natal

Co-Author 14: Mekgoe, 0

Co-Author 14 Institute: University of the Witwatersrand

Co-Author 15: Madhi, S

Co-Author 15 Institute: National Institute for Communicable Diseases (NICD)

Co-Author 16: Cohen, $C$

Co-Author 16 Institute: National Institute for Communicable Diseases (NICD)

E-mail Address: bwe3@cdc.gov

Country: South Africa

ABSTRACT TITLE: EPIDEMIOLOGY OF INFLUENZA AND RESPIRATORY SYNCYTIAL VIRUS (RSV)-ASSOCIATED HOSPITALISATION IN CHILDREN AGED

\section{Introduction}

Few data are available describing the spectrum of influenza- or respiratory syncytial virus (RSV)-associated hospitalised illness in children aged $<5$ years in sub-Saharan Africa.

\section{Methods}

During 2011-2016, we conducted prospective, hospital-based surveillance for severe respiratory illness (SRI) in children aged $<5$ years at two sites. In infants aged 2 days to $<3$ months, SRI was defined as a diagnosis of suspected sepsis or physician-diagnosed acute lower respiratory tract infection, irrespective of signs and symptoms and duration of illness. In children aged 3 to 59 months, SRI was defined as a physician-diagnosed lower respiratory tract infection, irrespective of signs and symptoms and duration of illness. Nasopharyngeal aspirates were tested for influenza and RSV by real-time reverse transcription polymerase chain reaction. We estimated rates of influenza- and RSV-associated hospitalised SRI. We examined clinical and epidemiological differences between 
children who tested positive for influenza versus RSV using multivariable penalised logistic regression.

\section{Results}

Among 3657 hospitalised children, 200 (5.5\%) tested positive for influenza viruses, 877 (24.0\%) for RSV, and 11 (0.3\%) for both (excluded from further analyses). The median age of children hospitalised with influenza was 14 months versus 4 months for RSV $(p<0.01)$. Influenza-associated hospitalisation rates per 100000 were highest among infants aged 6-11 months (524, 95\% Confidence Interval (Cl) 389-677), while RSV-associated hospitalisation rates were highest in infants aged 0-2 months (6 557, 95\% Cl 5 972-7 261). HIV-exposure was associated with increased incidence of influenza- and RSV-associated hospitalisation in infants aged 0-5 months, relative risk (RR) 2.2 and 1.5 respectively. HIVinfection was associated with increased incidence of influenza- and RSV-associated hospitalisation in all age groups, RR range 2.2-6.7.

\section{Conclusion}

Influenza- and RSV-associated hospitalisation were common among South African infants. HIV-infection and HIV-exposure increased risk of influenza- and RSV-associated hospitalisation in South African children.

\section{ID: 8638}

Category: Paediatric Infectious Diseases (SASPID)

Permission: Yes

Co-Author 1: Silvia, S

Co-Author 1 Institute: Private Practice

Co-Author 2: Frigati, L

Co-Author 2 Institute: Stellenbosch University, Tygerberg Hospital Co-Author 3: Goussard, P

Co-Author 3 Institute: Stellenbosch University, Tygerberg Hospital Co-Author 4: Schaaf, HS

Co-Author 4 Institute: Stellenbosch University

E-mail Address: frigati@sun.ac.za

Country: South Africa

\section{ABSTRACT TITLE: CULTURE-CONFIRMED TUBERCULOSIS IN INFANTS LESS THAN THREE MONTHS OF AGE: A CLINICAL DESCRIPTION OF 71 CASES}

\section{Introduction}

Young infants are particularly at risk for disseminated and severe pulmonary tuberculosis (TB). Few studies address TB disease in infants less than three months of age. The aim of this study was to describe clinical and radiological characteristics of TB in a cohort of infants in this age group.

\section{Methods}

We conducted a retrospective, descriptive study of all infants below three months (91 days) of age (on date of admission to hospital) with culture-confirmed TB on any clinical specimen. The infants were identified using a database of prospectively collected Mycobacterium tuberculosis-positive culture results for all children $<13$ years who presented to Tygerberg Hospital, a tertiary referral hospital in South Africa, from 1 March 2003 through 30 June 2011. Infants with mycobacterial disease caused by M. bovis BCG and nontuberculous mycobacteria were excluded

\section{Results}

Seventy-one infants met the inclusion criteria. Nineteen infants (27\%) were classified as having congenital TB. Sixty-six (92\%) had pulmonary TB, of which 22/66 (33\%) also had extrapulmonary TB; four (6\%) infants had only extrapulmonary TB. Twenty-seven infants (38\%) were HIV exposed and 10 infants (14\%) were HIV-infected. Five (7\%) infants had drug-resistant TB. Cough, loss of weight or failure to gain weight and history of fever were the most common presenting symptoms and were found in 41 (58\%), 29 (41\%) and 24 (34\%) of infants respectively. Eighteen (25\%), 15 (21\%) and $6(8 \%)$ infants presented with respiratory distress, wheezing and stridor, respectively. Chest $\mathrm{X}$-ray abnormalities included: mediastinal lymphadenopathy (39; 55\%), large airway compression (39, 55\%), alveolar/bronchopneumonic opacification (47; 66\%), miliary pattern $(14 ; 20 \%)$ and cavities $(9 ; 13 \%)$ infants. Of the 39 with airway compression, 21 (54\%) underwent bronchoscopy and 21 (54\%) infants required lymph node decompression. Ten (14\%) infants died.

\section{Conclusion}

Infants under three months of age presented with severe pulmonary manifestations and also disseminated disease.

\section{ID: 8294}

Category: Sexually Transmitted Diseases (STDSSA)

Permission: Yes

Authors:

\section{Author institutions/affiliations:}

Co-Author 1: le Roux, M

Co-Author 1 Institute: Sefako Makgatho Health Sciences University Co-Author 2: Mafunise, M

Co-Author 2 Institute: Sefako Makgatho Health Sciences University

Co-Author 3: de Villiers, B

Co-Author 3 Institute: Sefako Makgatho Health Sciences University Co-Author 4: Ditsele, M

Co-Author 4 Institute: National Health Laboratory Service,

Dr George Mukhari Academic Hospital

E-mail Address: marcelle.leroux@smu.ac.za

Country: South Africa

ABSTRACT TITLE: PREVALENCE AND MOLECULAR ANALYSIS OF MYCOPLASMA GENITALIUM STRAINS ISOLATED FROM PREGNANT WOMEN AT AN ACADEMIC HOSPITAL IN PRETORIA, SOUTH AFRICA 


\section{Introduction}

M. genitalium is sexually transmitted causing urethritis in men and cervicitis in women. Treatment is syndromically and detection of antimicrobial resistance and typing of strains relies on DNA sequence data. High levels of sequence variability between clinical isolates are seen which may be associated with antimicrobial resistance. This study was done to determine the prevalence and the molecular characteristics of $M$. genitalium strains from pregnant women attending the $\mathrm{Dr}$ George Mukhari Academic Hospital (DGMAH).

\section{Methods}

Endocervical swabs were collected from 100 pregnant women attending DGMAH. The specimens were screened for M. genitalium using a real-time PCR assay. Genotypic resistance markers for macrolide and fluoroquinolones were determined by sequence analysis of the V-region of the $23 \mathrm{~S}$ rRNA, gyrA, and parC genes respectively. The strains were typed using $\mathrm{mgpB}$ single-nucleotide polymorphism typing (SNP) and MG309 variable number tandem (VNTR) analysis.

\section{Results}

The prevalence of $M$. genitalium was $7.0 \%$. In two isolates macrolide resistance-associated mutations were seen. No resistanceassociated mutations were seen in the gyrA genes,but a parC fluoroquinolone resistance-associated mutation was seen in one of the macrolide resistant isolates. SNP typing revealed four sequence types, and four different types were also seen using MG309 VNTR analysis.Typing assigned M.genitalium to two major clusters with macrolide and fluoroquinolones resistance in the same cluster.

\section{Conclusion}

Mycoplasma genitalium is frequently undiagnosed. This study reported the first multidrug resistant $M$. genitalium strain in South Africa. As azithromycin was included in the national syndromic treatment guidelines in 2015 , it is alarming to already find resistance-associated genes.

\section{ID: 8399}

Category: Sexually Transmitted Diseases (STDSSA)

Permission: Yes

Co-Author 1: Ngwenya, I

Co-Author 1 Institute: Sefako Makgatho Health Sciences University Microbiology Department

Co-Author 2: le Roux, M

Co-Author 2 Institute: Sefako Makgatho Health Sciences University, Microbiology Department

Co-Author 3: de Villiers, B
Co-Author 3 Institute: Sefako Makgatho Health Sciences University, Microbiology Department

Co-Author 4: Ditsele, M

Co-Author 4 Institute: Sefako Makgatho Health Sciences University, Microbiology Department; National Health Laboratory Service Dr George Mukhari Academic Hospital

E-mail Address: ingweny@gmail.com

Country: South Africa

ABSTRACT TITLE: EVALUATION OF TWO COMMERCIAL NAATS FOR DETECTION OFC. TRACHOMATIS,

N. GONORRHOEAE AND T. VAGINALIS IN ENDOCERVICAL SWABS COLLECTED FROM PREGNANT WOMEN ATTENDING THE DGMAH

\section{Introduction}

Each year about 500 million new cases of sexually transmitted infections occur in men and women worldwide. Nucleic acid amplification tests offer a sensitive and specific diagnostic approach for the detection of STIs. The aim of the study was to evaluate two commercial nucleic acid amplification tests for the detection of Chlamydia trachomatis(CT), Neisseria gonorrhoeae (NG) and Trichomonas vaginalis (TV) in endocervical swabs.

\section{Methods}

One hundred endocervical swab samples that were collected from women attending the termination of pregnancy and ante-natal clinics at DGMAH were used. Multiplex detection of NG, CT and TV was done using the Real-time NG/CT/TV (Sacace, Italy) and Seegene Seeplex ACE Detection (Seegene, Korea) assays according to the manufacturers' instructions as well as by an in-house PCR. An expanded gold standard was used to evaluate the assays.

\section{Results}

Using the Sacace real time $\mathrm{m}-\mathrm{PCR}$, NG was detected in 5\%; $\mathrm{CT}$ in $24 \%$ and TV in $7 \%$ of the samples. Using Seegene Seeplex ACE Detection assay, NG was detected in $4 \%, \mathrm{CT}$ in $22 \%$ and TV in $4 \%$ of the specimens while using in-house PCR, NG was detected in $6 \%$, CT in $18 \%$ and TV in $9 \%$ of the samples.When applying the expanded gold standard the prevalence of the pathogens was as follows: N. gonorrhoeae $4 \%$; C. trachomatis $22 \%$ and T. vaginalis $5 \%$. The Seegene Seeplex assays performed the best in terms of detecting N. gonorrhoeae and C. trachomatis,followed by the Sacace real time assay. The in-house PCR was the most sensitive and specific in detecting T. vaginalis.

\section{Conclusion}

The three molecular assays used in this study were cost-effective and fairly sensitive and specific for the three pathogens. The two commercial assays were more successful at detecting N. gonorrhoeae and C. trachomatis while the in-house PCR best detected T. vaginalis. 


\section{FIDSSA 2017 Congress Abstracts Poster Presentations}

\section{ID: 8585}

Category: Infection Control (ICSSA)

Permission: Yes

Co-Author 1: le Roux, K

Co-Author 1 Institute: Department of Clinical Microbiology and Infectious Diseases, School of Pathology, University of the Witwatersrand, Faculty of Health Sciences, Infection Control, Charlotte Maxeke Johannesburg Academic Hospital

Co-Author 2: Singh, L

Co-Author 2 Institute: Department of Clinical Microbiology and Infectious Diseases, School of Pathology, University of the Witwatersrand, Faculty of Health Sciences, Infection Control, Charlotte Maxeke Johannesburg Academic Hospital

\section{Co-Author 3: Makola, P}

Co-Author 2 Institute: Department of Clinical Microbiology and Infectious Diseases, School of Pathology, University of the Witwatersrand, Faculty of Health Sciences, Infection Control, Charlotte Maxeke Johannesburg Academic Hospital

E-mail Address: karren.leroux@nhls.ac.za

Country: South Africa

\section{ABSTRACT TITLE: EVALUATION OF THE BD MAX FOR THE MULTIPLEX DETECTION OF MRSA, CRE AND ENTEROPATHOGENS}

\section{Introduction}

Multiplex qPCR has been introduced into the diagnosis of bacterial pathogens. Such assays produce rapid, sensitive and specific diagnosis and can identify multiple organisms simultaneously as compared to culture. In detecting multi-drug resistant organisms such as MRSA and CRE and in diagnosis of Enteric diseases, a rapid diagnosis allows for timeous infection control strategies and a better public health response. The BD MAX ${ }^{\mathrm{TM}}$ System is an open fully automated real time PCR instrument, which can also run different assays simultaneously. This study will evaluate 3 BD Max assays: the BD Max MRSA XT and Extended Enteric Bacterial Panel and the BioGx Assay for VIM, GES, OXA, NDM, KPC and mcr-1 (CRE Assay).

\section{Methods}

Positive and negative cultures for each assay were spiked into swabs or stool samples at $1 \times 104 \mathrm{CFU} / \mathrm{ml}$. Results were compared to culture (MRSA and Enteric) or a Roche LightMix real time PCR assay (CRE). Limits of detection were determined through 1:10 serial dilution of two positive samples per assay.

\section{Results}

All assays had accuracy, sensitivity, specificity, positive and negative predictive values of $100 \%$. The limits of detection for each assay were: $1 \times 104 \mathrm{CFU} / \mathrm{ml}$ (Enteric), $1 \times 103$ CFU/ml (MRSA) and $1 \times 102 \mathrm{CFU} / \mathrm{ml}$ (CRE) respectively. Preparation of samples took 2-10 minutes and in total the assays took $+/-2.5$ hours to run.

\section{Conclusion}

The BD Max is a simple, rapid, highly sensitive and specific system for detection of multiple bacterial pathogens with minimal expertise required. The MRSA assay also eliminates false positives in methicillin-resistant coagulase negative Staphylococcus and false negatives in mecC containing $S$. aureus. The CRE detects presence of several carbapenemases and colistin resistance but culture is still required for identification. Being an open system additional assays can be designed to run on one instrument. The BD Max is therefore a suitable platform especially in resource-limited laboratories.

\section{ID: 8692}

Category: Infectious Diseases (IDSSA)

Permission: Yes

Co-Author 1: McCarthy, $\mathrm{K}$

Co-Author 1 Institute: National Institute for Communicable Diseases

Co-Author 2: Ntshoe, G

Co-Author 2 Institute: National Institute for Communicable Diseases

Co-Author 3: Govender, N

Co-Author 3 Institute: National Institute for Communicable Diseases

Co-Author 4: Blumberg, L

Co-Author 4 Institute: National Institute for Communicable Diseases

E-mail Address: kerriganm@nicd.ac.za

Country: South Africa

ABSTRACT TITLE: OUTBREAK PREPAREDNESS AND THE NICD 24-HOUR HOTLINE - A REVIEW OF CALLS MADE TO THE HOTLINE, AUGUST 2016 TO JULY 2017

\section{Introduction}

The International Health Regulations (IHR) Core Capacity Monitoring framework requires public health services to maintain 24-hr availability for outbreak alert and response. The NICD hotline 
cellphone number is widely distributed to facilitate investigation of communicable diseases with public health importance. We review calls made to the NICD 24-hr hotline to assess adherence to IHR, technical content of calls made, and role of the hotline in South African outbreak responses.

\section{Methods}

Calls to the NICD hotline (082-883-9920) are documented using a standardised data collection tool by each doctor on call. Postcall alerts are defined as outbreaks/potential outbreaks, and risk assessment is graded using WHO Mass Gatherings risk characterisation as minimal, minor, moderate, major or severe. Summary statistics are generated for number and origin of alert/ call, type of enquiry, caller details, clinical details, diagnosis and turnaround time.

\section{Results}

From 1 July 2016 to 30 June 2017, 1083 calls were documented, with an average of 90 calls/month (standard deviation (SD) 3.57). Six-hundred-and-thirteen $(57 \%)$ originated from the private sector. The majority of callers were situated in Gauteng (409, $38 \%)$, KwaZulu-Natal $(294,27 \%)$ and Western Cape provinces (167, 15\%) respectively. Calls pertained to rabies post-exposure prophylaxis (PEP) $(484,45 \%)$, requests for assistance with diagnosis of infectious diseases in individual patients (180, 16\%), PEP for nonrabies conditions $(139,13 \%)$, and advice regarding public health management for laboratory-confirmed conditions $(117,11 \%)$ or other reason $(162,15 \%)$. Of 878 calls with a risk characterisation, $50(6 \%)$ alerts were identified as 'outbreaks', 18 (2\%) events attained 'moderate' risk status and 15 deaths were reported. Food-borne illness were the most common outbreak leading to an alert, (21/50) followed by viral haemorrhagic fever (5).

\section{Conclusion}

The NICD 24-hr hotline contributes to adherence to IHR, and facilitates outbreak alert and response activities.

\section{ID: 8338}

Category: Infectious Diseases (IDSSA)

Permission: Yes

Co-Author 1: Kalule, JB

Co-Author 1 Institute: University of Cape Town, Department of Pathology; National Institute for Communicable Disease; University of Cape Town

Co-Author 2: Keddy, K

Co-Author 2 Institute: University of Cape Town, Department of Pathology; National Institute for Communicable Disease; University of Cape Town

Co-Author 3: Nicol, MN

Co-Author 3 Institute: University of Cape Town, Department of Pathology; National Institute for Communicable Disease; University of Cape Town

E-mail Address: kaluleb@gmail.com

Country: South Africa
ABSTRACT TITLE: PREVALENCE AND

CHARACTERISATION OF FOOD AND WATERBORNE

BACTERIAL PATHOGENS IN RIVER WATER, MEAT FROM

INFORMAL ABATTOIRS, AND STOOL FROM CHILDREN

WITH DIARRHOEA IN NYANGA TOWNSHIP, CAPE TOWN, SOUTH AFRICA

\section{Introduction}

Morbidity and mortality due to diarrhoeal diseases is heaviest among children in sub-Saharan Africa and south-east Asia, especially in areas with poor hygiene. Informal settlements face hygiene challenges and have high infant mortality rates; yet little is known about the contribution of foodborne diarrhoeic pathogens. We aimed to determine the prevalence and characteristics of bacterial pathogens in the stool of children with diarrhoea, river water, and meat from informal abattoirs in Nyanga. Also, to assess the level of faecal contamination of the river water.

\section{Methods}

Samples were collected between September 2015 and May 2016 in Nyanga, Cape Town. Clinical and epidemiological data were collected using a questionnaire. A duplex real-time PCR assay, gelbased PCR, and CHROMagarTMSTEC were used to screen Tryptic Soy Broth enrichments of the samples for stx, rfbE and wbdl, and STEC respectively. The enrichments (or sample) were also tested for selected foodborne pathogens using standardised methods. The resultant bacterial pathogens were serotyped (Salmonella and Shigella), tested for virulence genes, and antibiotic susceptibility determined.

\section{Results}

A total of 64,66 , and 85 water, stool, and meat samples respectively were collected. Diffusely adherent E. coli (DAEC-18\% [95\% Confidence Interval $=11-29])$, and Shigella flexneri $(17 \%[95 \% \mathrm{Cl}=9-27])$, were most prevalent in the stool of children (mean age $=14.9$ months) that presented with acute diarrhoea (mean duration $=2.5$ days) with one or more pathogens detected in $73 \%$ (48/66) of children. Other pathogens in stool included Salmonella (6\%), Plesiomonas (9\%), Aeromonas (3\%), and Campylobacter (5\%). Meat carried Salmonella enterica (5\%), Aeromonas sorbria (3\%), Campylobacter jejuni (5\%), and diarrhoeic E. coli (3\%).Water was highly contaminated (mean $\mathrm{CFU} / \mathrm{ml}=1.11 \mathrm{E}+06-2.74 \mathrm{E}+05$ ) and carried Plesiomonas shigelloides (17\%), Shigella flexneri (5\%), Vibrio vulnificus (9\%), and diarrhoeic E. coli $(7 \%)$. Trimethoprim-sulphamethoxazole resistance was observed amongst all pathogens, irrespective of their source.

\section{Conclusion}

Foodborne pathogens were prevalent in human and non-human sources in this informal settlement. Local and international public health standards should be implemented, and further larger scale research is needed to fully define the prevalence of foodborne pathogens in similar settings in South Africa. 


\section{ID: 8363}

Category: Infectious Diseases (IDSSA)

Permission: Yes

Co-Author 1: Rampersad, T

Co-Author 1 Institute: University of KwaZulu-Natal

Co-Author 2: Sobia, P

Co-Author 2 Institute: University of KwaZulu-Natal

E-mail Address: Tashmin.r@gmail.com

Country: South Africa

\section{ABSTRACT TITLE: THE DEVELOPMENT AND APPLICATION OF A HIGH THROUGHPUTMETHODOLOGY TO DETERMINE MICS OF MYCOBACTERIUM TUBERCULOSIS ISOLATES AGAINST ANTIMICROBIAL AGENTS}

\section{Introduction}

Drug susceptibility testing of Mycobacterium tuberculosis is time consuming and expensive. Multi-point inoculation offers the advantage of testing multiple isolates on a series of solid media with a single break-point concentration of a drug in each plate or a series of different drug concentrations of one drug. The former provides the resistance profile of the isolates tested while the latter results in an MIC. This has the potential to reduce consumables and labour costs for break-point susceptibility testing as well as MIC determination. Although multi-point inoculation had been performed with other bacterial species, it has hardly been used with $\mathrm{M}$. tuberculosis since its slow growth and clumping has been thought to be factors to influence reproducibility of this method adversely. We aimed to determine the reproducibility of MIC determination for anti-TB drugs of M. tuberculosis isolates using agar dilution with multi-point inoculation, and thereafter validating the results by comparing it to classic agar dilution on quadrant plates and the MTT assay.

\section{Methods}

Thirty M. tuberculosis isolates were grown in Middlebrook 7H9 broth with 20\% Tween until mid-log phase was reached. Agar dilution MICs were determined on Middlebrook 7H10 agar for 11 anti-TB drugs at concentrations ranging from 128 to $0.125 \mathrm{mg} / \mathrm{L}$. The agar plates were inoculated using a multi-point inoculation device with 36 points each delivering 1 micro litre of a suspension of $1 \times 104 \mathrm{cfu} / \mathrm{ml}$. For the quadrant plate method and the MTT assay 100 micro litre of the same suspension was used. All tests were done in triplicate.

\section{Results}

Agar dilution with multi-point inoculation was found to be reproducible within the 11 anti-TB drugs tested and correlated well with agar dilution on quadrant plates and the MTT assay for the three anti-TB drugs tested.

\section{Conclusion}

The multi-point inoculation method has potential for wide scale application in break-point drug susceptibility testing as well as MIC testing of M. tuberculosis isolates.

\section{ID: 8396}

Category: Infectious Diseases (IDSSA)

Permission: Yes

Co-Author 1: Steinhaus, N

Co-Author 1 Institute: University of Cape Town; Groote Schuur Hospital

Co-Author 2: Al-talib, M

Co-Author 2 Institute: University of Cape Town; Groote Schuur Hospital

Co-Author 3: Bamford, C

Co-Author 3 Institute: University of Cape Town; Groote Schuur Hospital

Co-Author 4: Ive, P

Co-Author 4 Institute: University of Cape Town; Groote Schuur Hospital

Co-Author 5: Mendelson, M

Co-Author 5 Institute: University of Cape Town; Groote Schuur Hospital

Co-Author 6: Davies, M

Co-Author 6 Institute: University of Cape Town; Groote Schuur Hospital

Co-Author 7: Wasserman, $\mathrm{S}$

Co-Author 7 Institute: University of Cape Town; Groote Schuur Hospital

E-mail Address: stnnic017@myuct.ac.za

Country: South Africa

\section{ABSTRACT TITLE: THE MANAGEMENT AND OUTCOMES OF STAPHYLOCOCCUS AUREUS BACTERAEMIA AT A SOUTH AFRICAN REFERRAL HOSPITAL: A PROSPECTIVE OBSERVATIONAL STUDY}

\section{Introduction}

Staphylococcus aureus bacteraemia (SAB) is an important cause of mortality and morbidity. Limited evidence exists on the management and outcomes of $S A B$ in a South African setting. The aim of this study is to describe a local cohort of patients with $\mathrm{SAB}$, assess outcomes, and explore the factors associated with complications and death.

\section{Methods}

We conducted a prospective observational study of all patients over the age of 13 years admitted to Groote Schuur Hospital with SAB. The following data were collected: demographics and medical comorbidities, management, and 90-day outcomes. We summarised patient characteristics and examined factors associated with poor outcomes using multivariate logistic regression. 


\section{Results}

One hundred consecutive distinct SAB infection episodes, in 98 patients, were included between November 2013 and January 2015; median age 50 years (interquartile range (IQR) 33 to 63 ), males $72 \%$, HIV infection $21.5 \%$. Median time to notification of culture results was 51.5 hours (IQR 41.0 to 66.8). SAB was healthcare-associated in $68.4 \%$, with $44.7 \%$ linked to drip-site infection; $24.0 \%$ of cases were caused by methicillin-resistant $S$. aureus(MRSA). Ninetyday mortality was $47 \%$, with $83.3 \%$ of deaths attributable to $S A B$. Independent prognostic factors for 90-day mortality were MRSA infection (OR 3.1; 95\% confidence interval (CI) 1.1 to 9.7) and the presence of co-morbidities (OR 4.8; $95 \% \mathrm{Cl} 1.5$ to 19.8); the risk for complicated infection was higher with suboptimal or inadequate antibiotic therapy (OR 7.2; 95\% Cl 1.6 to 39.9). Definitive antibiotic therapy was suboptimal or inadequate in $22.6 \%$ of all cases, and in $35.3 \%$ of those with MRSA

\section{Conclusion}

Most episodes of SAB were healthcare associated and related to drip-site infection, suggesting that many of these infections are preventable. MRSA rates and overall SAB mortality were higher than in high income countries. Our findings suggest that there is a need for improved management.

\section{ID: 8443}

Category: Infectious Diseases (IDSSA)

Permission: Yes

Co-Author 1: Annear, D

Co-Author 1 Institute: Department of Biochemistry and Microbiology, Nelson Mandela University, Port Elizabeth, South Africa

Co-Author 2: Gadia, R

Co-Author 2 Institute: Jose Pearson TB Hospital, Eastern Cape Department of Health, Port Elizabeth, South Africa

Co-Author 3: Black, J

Co-Author 3 Institute: Department of Medicine, Livingstone Hospital, Eastern Cape Department of Health, Port Elizabeth, South Africa

Co-Author 4: Truter, I

Co-Author 4 Institute: Drug Utilisation Research Unit (DURU), Department of Pharmacy, Nelson Mandela University, Port Elizabeth, South Africa

\section{Co-Author 5: Bamford, C}

Co-Author 5 Institute: National Health Laboratory Service, Groote Schuur Microbiology and Division of Medical Microbiology, University of Cape Town, Cape Town, South Africa

Co-Author 6: Govender, S

Co-Author 6 Institute: Department of Biochemistry and Microbiology, Nelson Mandela University, Port Elizabeth, South Africa
E-mail Address: dale.annear@gmail.com

Country: South Africa

\section{ABSTRACT TITLE: INCIDENCE OF BACTERIAL INFECTIONS AND COLONISATION IN PATIENTS ADMITTED TO A TUBERCULOSIS HOSPITAL}

\section{Introduction}

Hospital-acquired infections are a worldwide problem, which poses an extreme threat to public health. Patients with drug-resistant tuberculosis (TB) are treated with multiple antibiotics including moxifloxacin, linezolid, and meropenem. Broad spectrum antibiotic exposure and prolonged hospital admission puts them at greater risk for colonisation by multi-drug resistant (MDR) bacteria. The aim of the study was to determine the spectrum of bacterial colonisation in TB patients upon admission and during hospitalisation.

\section{Method}

Nasal, groin and rectal swabs (for the detection of EBSLs, carbapenem-resistant Enterobacteriaceae (CRE), vancomycinresistant enterococci (VRE) and MRSA) were analysed from a cohort of patients [ $n=37: 28$ (community), nine (healthcare facilities)] admitted to a TB hospital upon admission and at four-week intervals thereafter during hospitalisation. Identification and antimicrobial susceptibility testing of bacterial isolates $(n=64)$ were determined at National Health Laboratory Services (NHLS) by the VITEK-MS system. PCR and DNA sequencing were used for detection of carbapenem resistance genes.

\section{Result}

Patients ( $n=13 / 37 ; 35 \%$ ) were colonised by an MDR pathogen (ESBL, MRSA) on admission. Colonisation rates were lower in patients admitted from the community $(9 / 28 ; 32 \%)$ compared to those transferred from other healthcare facilities $(4 / 9 ; 44 \%)$. All patients from the community $(17 / 37 ; 46 \%$ of total patients) became colonised by MDR bacteria within one month of admission, mostly with ESBL producing Enterobacteriaceae. Only two patients had MRSA colonisation at admission. Among ESBL, Enterobacteriacae, Escherichia coli $(42 / 64 ; 65.6 \%)$ and Klebsiella pneumoniae (15/64; 23.4\%) predominated. Two Enterobacteriacae isolates with reduced carbapenem susceptibility did not contain carbapenemaseencoding genes. Nineteen percent (7/37) of patients demised during their hospitalisation, however no deaths could be attributed to drug-resistant organisms.

\section{Conclusion}

This study provides insight into the spectrum of bacterial pathogen colonisation in hospitalised TB patients and suggests a review of infection control programmes and practices at the TB hospital.

\section{ID: 8696}

Category: Infectious Diseases (IDSSA)

Permission: Yes

Co-Author 1: Govender, N 
Co-Author 1 Institute: National Institute for Communicable Diseases

Co-Author 2: Ntshoe, G

Co-Author 2 Institute: National Institute for Communicable Diseases

Co-Author 3: McCarthy, K

Co-Author 3 Institute: National Institute for Communicable Diseases

E-mail Address: nevashang@nicd.ac.za

Country: South Africa

\section{ABSTRACT TITLE: LESSONS LEARNT FROM AN ACTIVATION OF THE NATIONAL EMERGENCY OPERATIONS CENTRE, SOUTH AFRICA, 2017}

\section{Introduction}

In response to the western African Ebola crisis of 2014, the World Health Organization recommended that all State Parties should develop, strengthen and maintain their capacity to respond promptly and effectively to public health risks and public health emergencies of international concern. To address public health emergencies national and subnational health authorities require efficient and coordinated responses from competent and skilled staff in equipped facilities, supported by appropriate protocols and training. Via a memorandum of agreement (MOA) with the National Department of Health, an Emergency Operations Centre (EOC) was developed at the National Institute for Communicable Diseases (NICD).

\section{Methods}

To test the preliminary protocols and systems of the EOC - in the absence of a large-scale infectious disease outbreak - the NICD accepted the responsibility of activating the EOC in response to a public health emergency of a non-infectious nature. The activation was governed by an addendum to the MOA stipulating that the NICD should assist a provincial health department in managing the public health emergency by providing operational support. For this purpose the NICD implemented an Incident Management System (IMS).

\section{Results}

The NICD provided support in the form of incident management, financial administration, logistics, human resources, data management and communications. Challenges identified through the activation resulted in the following key lessons learnt: response teams must be flexible, knowledgeable and willing to work within an IMS; decision-makers to be available and involved to manage risks; establishment of clear lines of communication helps define roles and responsibilities; strong leadership within the IMS is required to coordinate teams.

\section{Conclusion}

Overall, the NICD satisfied the requirements of the MOA. However, in preparation for a public health emergency of an infectious nature, so as to rapidly reduce morbidity and mortality, it is recommended that universal training of infectious disease control practitioners on IMS and its application be prioritised.

\section{ID: 8530}

Category: Sexually Transmitted Diseases (STDSSA)

Permission: Yes

Co-Author 1: Singh, R

Co-Author 1 Institute: National Health Laboratory Service

Co-Author 2: Garrett, N

Co-Author 2 Institute: Centre for the Aids Programme of Research in South Africa

Co-Author 3: Ramsuran, V

Co-Author 3 Institute: University of KwaZulu-Natal

Co-Author 4: Mitchev, N

Co-Author 4 Institute: National Health Laboratory Service

Co-Author 5: Kajee, A

Co-Author 5 Institute: National Health Laboratory Service

Co-Author 6: Khanyile, T

Co-Author 6 Institute: National Health Laboratory Service

Co-Author 7: Ramlal, P

Co-Author 7 Institute: National Health Laboratory Service

Co-Author 8: Ngcapu, S

Co-Author $\mathbf{8}$ Institute: Centre for the Aids Programme of Research in South Africa

Co-Author 9: Mlisana, K

Co-Author 9 Institute: National Health Laboratory Service

E-mail Address: singhra@ukzn.ac.za

Country: South Africa

\section{ABSTRACT TITLE: PROFILING VAGINAL MICROBIOTA USING SENSITIVE QUANTIFICATION ASSAYS}

\section{Introduction}

Bacterial vaginosis (BV) is the most common cause of vaginal discharge syndrome affecting up to $60 \%$ of women of childbearing age in parts of South Africa. Diagnosis of BV in resource-poor settings is not prioritised and relies on semi-quantitative microscopy algorithms such as Amsel's criteria and Nugent score (NS). Here, we evaluated a quantitative real-time PCR (qPCR) assay to detect bacterial communities.

\section{Methods}

Vaginal swabs from 251 South African women presenting with STI symptoms were evaluated for BV using NS. By qPCR, we analysed DNA from vaginal swabs for six BV-associated bacteria, using ThermoFisher vaginal microbiota panel: Gardnerella vaginalis(GV), Prevotella bivia (PB), BV-associated-bacteria-2 (BVAB2), Megasphaera 1(M-1), Lactobacillus crispatus(LC) and Lactobacillus jensenii (LJ), and determined quantitative bacterial levels. The sensitivity and 
specificity of each qPCR assay, compared to NS, was measured using ROC analysis. Cut-offs were calculated based on sensitivities and specificities. A logistic regression model was used to determine the strongest predictors of BV status for all bacterium.

\section{Results}

Nugent scores from 251 women showed 30.2\% normal flora (NS $0-3$ ), 34.2\% intermediate flora (NS 4-6) and 36.3\% BV (NS > 7). The prevalence of BVAB2, GV, M-1, PB, GBM 46.2\%, 68.1\%, 31.4\%, 47.4\% and $60.1 \%$, respectively. Combined titres of GV, BVAB2 and $M-1$, (GBM) generated a greater Area under curve (AUC), 0.9, compared to individual organisms $(0.68,0.74$ and 0.79 , respectively). The cutoff, sensitivity and specificity were as follows (listed respectively): BVAB2 (> 0.006; 72\%; 73\%), GV (> 12.43; 95\%; 70\%), M-1 (> 0.18; $64 \% ; 92 \%), \mathrm{PB}(>0.02 ; 52 \% ; 67 \%), \mathrm{GBM}(>514 ; 94 \% ; 77 \%)$ (all $p<0.05)$. Furthermore, an increase in GBM levels $(p=0.034$ ), decrease in $L C(p<0.0001)$ and $L J(p=0.026)$ were identified as the strongest predictors of BV status.

\section{Conclusion}

We developed an accurate assay, which has the potential to be used as a BV diagnostic and monitoring tool.

\section{ID: 8505}

Category: Antimicrobial Resistance (SAASP)

Permission: Yes

Co-Author 1: Singh-Moodley, A

Co-Author 1 Institute: National Institute for Communicable Diseases/National Health Laboratory Services

Co-Author 2: Perovic, 0

Co-Author 2 Institute: National Institute for Communicable Diseases/National Health Laboratory Services

E-mail Address: mokgadir@gmail.com

Country: South Africa

\section{ABSTRACT TITLE: GENOTYPIC CHARACTERISATION OF AMPC B-LACTAMASES AMONG KLEBSIELLA PNEUMONIAE ISOLATED FROM PATIENTS WITH BACTERAEMIA IN SOUTH AFRICA}

\section{Introduction}

AmpC- $\beta$ lactamases are cephalosporinases produced by Enterobacteriaceae and confer resistance to $\beta$-lactam antibiotics. The distribution of plasmid-mediated $\mathrm{AmpC}$ resistance in South Africa is unknown. AmpC-producing Klebsiella pneumoniae acquire resistance through the plasmid-mediated ampC gene or hyperproduction of an inducible chromosomal AmpC enzyme. Due to limited specificity of phenotypic methods in detecting AmpC enzymes, there is a need to use molecular methods to accurately characterise $\mathrm{AmpC}$-mediated resistance. The aim of the study was to investigate the presence of plasmid-mediated AmpC $\beta$-lactamases in multi-drug resistant Klebsiella pneumoniae clinical isolates.

\section{Methods}

Klebsiella pneumoniae isolates were obtained from sentinel site laboratories as part of a surveillance programme from July 2010July 2012. Identification and antimicrobial susceptibility testing (AST) patterns of organisms were confirmed on VITEK ${ }^{\oplus}$ II system and the MicroScan ${ }^{\circledR}$ Walkaway system, respectively. Isolates that were non-susceptible to cefoxitin were selected for screening of genes coding for plasmid-mediated AmpC $\beta$-lactamase production (blaMOX, blaFOX, blaDHA, blaACC, blaEBC and blaClT) using a conventional PCR assay.

\section{Results}

Of 2774 Klebsiella pneumoniae isolates, 114 (4.1\%) were phenotypically resistant to cefoxitin and eligible for AmpC screening. AST results showed resistance to multiple antibiotics, including ceftazidime (94\%), cefepime (92\%), cefotaxime (89\%), cefoxitin (87\%), amoxycillin-clavulanate (89\%), trimethoprimesulfamethaxazole (91\%), gentamycin (93\%) and ciprofloxacin (82\%), but high susceptibility to imipenem and meropenem. Plasmidmediated AmpC $\beta$-lactamases were present in 8.8\% (10/114) of the isolates, with $5.3 \%$ of the resistance mediated by blaCIT $(n=6)$, and $3.5 \%$ by blaDHA $(n=4)$. A high percentage of isolates did not express the plasmid-mediated AmpC genes tested for (91.2\% $n=104)$.

\section{Conclusion}

Plasmid-mediated AmpC $\beta$-lactamases were present in $8.8 \%$ of Klebsiella pneumoniae isolates; these consisted of blaCIT and blaDHA. Further investigations are underway to characterise chromosomal hyper-producers in these isolates.

\section{ID: 8510}

Category: Antimicrobial Resistance (SAASP)

Permission: Yes

Co-Author 1: Moloto K

Co-Author 1 Institute: Department of Medical Microbiology, Faculty of Health Sciences, University of Pretoria

\section{Co-Author 2: Pitout JDD}

Co-Author 2 Institute: Department of Medical Microbiology, Faculty of Health Sciences, University of Pretoria; University of Calgary

\section{Co-Author 3: Ehlers MM}

Co-Author 3 Institute: Department of Medical Microbiology, Faculty of Health Sciences, University of Pretoria; Department of Medical Microbiology, Tshwane Academic Division, National Health Laboratory Service

Co-Author 4: Ismail F

Co-Author 4 Institute: Department of Medical Microbiology, Faculty of Health Sciences, University of Pretoria; Department of Medical Microbiology, Tshwane Academic Division, National Health Laboratory Service; National Institute for Communicable Diseases

Co-Author 5: Kock MM 
Co-Author 5 Institute: Department of Medical Microbiology, Faculty of Health Sciences, University of Pretoria; Department of Medical Microbiology, Tshwane Academic Division, National Health Laboratory Service

E-mail Address: kedisaletse05@gmail.com

Country: South Africa \& Canada

\section{ABSTRACT TITLE: PREVALENCE OF CARBAPENEMASE PRODUCING PSEUDOMONAS AERUGINOSA CIRCULATING IN PRETORIA, SOUTH AFRICA}

\section{Background}

Pseudomonas aeruginosa is an important nosocomial pathogen. Carbapenems are used for treating infections caused by multidrugresistant $P$. aeruginosa. The extensive use of carbapenems has led to the emergence of carbapenem resistance in this bacterium. Carbapenemases hydrolyse carbapenems efficiently and are considered the most clinically significant mechanism of carbapenem resistance in $P$. aeruginosa isolates. The aim of this study was to determine the rate of carbapenem resistance and virulence in $P$. aeruginosa circulating in the Pretoria region.

\section{Material and Methods}

A total of 237 consecutive non-repeat isolates were collected from the diagnostic division of the Department of Medical Microbiology (TAD, NHLS) from May to September 2016. Identification and susceptibility were determined by the VITEK ${ }^{\oplus}$ automated system. All imipenem-resistant isolates were screened for the presence of metallo-beta-lactamases using the MBL E-test. The following carbapenemases were detected using multiplex PCR assays: Verona integron encoded metallo-beta-lactamase, Oxacillinase-48, Adelaide imipenemase, German imipenemase, Sao Paulo metallobeta-lactamase, Imipenem metallo-beta-lactamase, New Dehli metallo-beta-lactamase and Guiana extended spectrum genes and selected virulence genes.

\section{Results}

Sixty (24\%) of the 237 P. aeruginosa isolates collected were reported as imipenem resistant. Fifteen [15/60] (25\%) imipenem-resistant isolates were confirmed as MBL positive. The blaVIM gene was detected in eight $(53 \%)$ of the fifteen MBL positive isolates. The remaining seven (47\%) MBL positive isolates harboured the GES gene. The NDM gene was detected in fourteen (93\%) of the15 MBL positive isolates. The OXA-48 and GES genes were detected from the imipenem-resistant isolates with percentages of $22 \%$ and $78 \%$, respectively. A high prevalence of quorum-sensing genes i.e. rhll (94\%), lasL (93\%) and lasR (92\%) were detected, while the effector enzyme (exoY) and phenazine (phzl) genes were detected at $0.4 \%$

\section{Conclusions}

The high prevalence of carbapenem resistant and carbapenemase producing $P$. aeruginos $a$ isolates found in this study highlights the need for alternative treatment options. Quorum sensing is important for regulating antimicrobial production and biofilm formation, enabling persistence and spread of resistant $P$. aeruginosa. There is an urgent need for the implementation of active surveillance systems and adequate infection control measures in South African hospitals to prevent and limit the spread of resistant bacteria

\section{ID: 8313}

Category: Sexually Transmitted Diseases (STDSSA)

Permission: Yes

Co-Author 1: Ditsele, M

Co-Author 1 Institute: National Health Laboratory Service, Dr George Mukhari Academic Hospital

Co-Author 2: le Roux, M

Co-Author 2 Institute: Sefako Makgatho Health Sciences University

Co-Author 3: Nemarude, L

Co-Author 3 Institute: Sefako Makgatho Health Sciences University

Co-Author 4: de Villiers, B

Co-Author 4 Institute: Sefako Makgatho Health Sciences University

Co-Author 5: Ngwenya, I

Co-Author 5 Institute: Sefako Makgatho Health Sciences University Co-Author 6: Nchabeleng, $M$

Co-Author 6 Institute: National Health Laboratory Service, Dr George Mukhari Academic Hospital; Sefako Makgatho Health Sciences University

E-mail Address: molebogeng.ditsele@nhls.ac.za

Country: South Africa

ABSTRACT TITLE: SEXUALLY TRANSMITTED INFECTIONS AMONG MEN HAVING SEX WITH MEN IN GA-RANKUWA, SOUTH AFRICA

\section{Introduction}

The prevalence of sexually transmitted infections (STIs) among men having sex with men (MSM) is high. Infections also occur in extragenital sites including the rectum and pharynx. Patients with STIs have a high risk of acquiring HIV and vice versa. This study was done to determine the prevalence of sexually transmitted pathogens in genital and extra-genital sites of MSM in Ga-Rankuwa.

\section{Methods}

Swabs (genital, oral and rectal) were collected from 175 consenting MSM. Multiplex real-time PCR was done to determine the presence of Neisseria gonorrhoeae (NG), Chlamydia trachomatis (CT), Mycoplasma genitalium (MG) and Trichomonas vaginalis (TV) in all specimens.

\section{Results}

One or more pathogen was detected in 65 (37.1\%) of the participants, of which CT was detected most frequently (22.9\%), followed by NG (16.6\%), MG (6.9\%) and TV (6.9\%). Most pathogens were detected in the rectal specimens (27.4\%) followed by genital (17.1\%) and oral (16.0\%) specimens. NG was detected most frequently in more than one specimen type, i.e. 43 isolates in 29 patients, followed by CT (40 isolates in 35 participants) and TV (12 isolates in 11 participants). 


\section{Conclusion}

There was a high prevalence of STls among this group of men, especially in the extra-genital sites. As MSM are at a high risk for STDs, including HIV infection, they should be counselled about repeated STI screening (including extra-genital sites) and safer sex. Public health messages aimed at MSM need to emphasise safe insertive as well as receptive sexual practices.

\section{ID: 8463}

Category: Sexually Transmitted Diseases (STDSSA)

Permission: Yes

Co-Author 1: Rambharose, $S$

Co-Author 1 Institute: University of KwaZulu-Natal

Co-Author 2: Kalhapure, RS

Co-Author 2 Institute: University of Texas at El Paso

Co-Author 3: Jadhav, M

Co-Author 3 Institute: University of KwaZulu-Natal

Co-Author 4: Govender, $T$

Co-Author 4 Institute: University of KwaZulu-Natal

E-mail Address: rambharoses@ukzn.ac.za

Country: South Africa \& United States of America

\section{ABSTRACT TITLE: NOVEL MONO, DI AND TRI-FATTY ACID ESTERS BEARING B-ALANINE T-BUTYL ESTER HEAD GROUPS ENHANCE THE TRANSDERMAL DELIVERY OF TENOFOVIR}

\section{Introduction}

Oral antiretroviral (ARV) drug therapy has significantly improved the treatment of HIV and AIDS. Innovative drug delivery strategies such as novel drug delivery systems and alternate routes of drug administration can overcome the limitations of oral ARV therapy. The use of chemical permeation enhancers (CPEs) has widened the pool of drugs that can be delivered via the transdermal route. This study explored the synthesis and characterisation of novel mono, di and tri-fatty acid ( $F A$ ) esters bearing $\beta$-alanine t-butyl ester head group as permeation enhancers for the transdermal delivery of tenofovir (TNF).

\section{Methods}

The novel esters were characterised using FTIR, 13C NMR, 1H NMR and HRMS. The biosafety of the novel FA esters was established using in vitro cell culture. In vitro transdermal permeation studies were performed using rat skin and Franz diffusion cells. Transepithelial electrical resistance (TEER) was used to establish the integrity of the treated skin. Hematoxylin and eosin (H\&E) stained images were used to histologically evaluate the treated skin samples.

\section{Results}

The synthesised compounds were non-toxic to mammalian cells confirming their safety for pharmaceutical applications. All the synthesised derivatives displayed better transdermal permeation enhancement capabilities as compared to their respective individual FAs. The mono oleate derivative (MOAPE) displayed the greatest ER for TNF (5.87) at 1\% w/w. Histological investigations of skin treated with MOAPE revealed fluidisation of the stratum corneum. Histological and TEER studies corroborated with the findings of the in vitro permeation experiments and revealed that there was no significant change to the viable epidermis of the skin after 1\% MOAPE exposure. The TEER findings also suggested that the permeation enhancement effects of MOAPE were not permanent, with the results indicating a return towards original skin integrity after removal of the enhancer formulation.

\section{Conclusion}

This study reported novel FA esters showing promise as effective permeation enhancers. The findings show that the novel mono ester derivative of OA (MOAPE) adds to the pool of CPEs available to formulation scientists and can be safely incorporated into TNF TDD systems

\section{ID: 8541}

Category: Sexually Transmitted Diseases (STDSSA)

Permission: Yes

Co-Author 1: Khanyile, $T$

Co-Author 1 Institute: University of KwaZulu Natal, College of Health Science, School of Laboratory Medicine and Medical Science

Co-Author 2: Mlisana, $\mathrm{K}$

Co-Author 2 Institute: Department of Microbiology, National Health Laboratory Services, KZN Academic Complex, Inkosi Albert Luthuli Central Hospital, Durban, South Africa; School of Laboratory Medicine

Co-Author 3: Singh, R

Co-Author 3 Institute: Department of Microbiology, National Health Laboratory Services, KZN Academic Complex, Inkosi Albert Luthuli Central Hospital, Durban, South Africa

Co-Author 4: Garrett, N

Co-Author 4 Institute: Centre for The AIDS Programme of Research in South Africa

Co-Author 5: Mitchev, N

Co-Author 5 Institute: University of KwaZulu Natal, College of Health Science, School of Laboratory Medicine and Medical Science

E-mail Address: thobilekhanyile45@yahoo.com

Country: South Africa

ABSTRACT TITLE: MOLECULAR CHARACTERISATION OF CHLAMYDIA TRACHOMATIS ISOLATES USING SEQUENCE VARIATION IN THE MAJOR OUTER MEMBRANE PROTEIN GENE (OMP1)

\section{Introduction}

We have applied High Resolution Melting Analysis (HRMA) for the genotyping of the Chlamydia trachomatis (CT) and applied 
it specifically to the 14 sexually transmitted infection-related genotypes: A-C, D-K, and L1-L3. Based on the genotype of the OMP1 (Outer Membrane Protein) gene CT is grouped into different serovars, which present in different clinical manifestations; with type $\mathrm{A}, \mathrm{B}, \mathrm{Ba}$, and $\mathrm{C}$ causing trachoma, D-K cause urogenital infections and LI, LII \& LIII associated with lymphogranuloma venereum (LGV). The OMP1 is one of the conserved genes found in CT. The aim of this study is to evaluate the prevalence of the above mentioned serovars.

\section{Methods}

Two hundred and sixty-five EswabTM clinical samples were screened for CT using Anyplex ${ }^{\mathrm{TM}}$ II STI-7 Detection. We confirmed the presence of the OMP1 gene with the conventional PCR. HRMA was performed to identify the CT serovars on a Quantstudio 5 qPCR instrument and $\mathrm{CDC}$ controls were included in the analysis. HRM analysis was done on the High-Resolution Melt Software v3.1.

\section{Results}

Using HRM we identified the following serovars: $A, B, C, D, E, F, G, I, J$, $L 3$. The highest prevalent serovars were serovar $F=29 \%$; $E=16.1 \%$; $I=12.9 \%$; $D$ and $G$ both $=9.7 \%$ and the rest below $6.5 \%$. No serovars: $\mathrm{H}, \mathrm{K}, \mathrm{L1}, \mathrm{L} 2$ were identified. These are the preliminary results which should be validated using sequencing to confirm each genotype.

\section{Conclusion}

In conclusion, serovars from D-K in this study dominate, with F being the most prevalent one. This serovar set has been associated with urogenital infections. HRM genotyping could be used to differentiate multiple concurrent urogenital $\mathrm{CT}$ infections in the clinical samples.

\section{ID: 8205}

Category: Antimicrobial Resistance (SAASP)

Permission: Yes

Co-Author 1: Singh, $S$

Co-Author 1 Institute: University of Kwa-Zulu Natal

Co-Author 2: Essack, $S$

Co-Author 2 Institute: University of Kwa-Zulu Natal

E-mail Address: shanaysingh87@gmail.com

Country: South Africa

\section{ABSTRACT TITLE: ANTIMICROBIAL RESISTANCE AND ANTIBIOTIC STEWARDSHIP: KNOWLEDGE, ATTITUDES AND PERCEPTIONS AMONGST FINAL-YEAR UNDERGRADUATE HEALTH PROFESSIONAL STUDENTS IN A SOUTH AFRICAN UNIVERSITY}

\section{Introduction}

Antimicrobial resistance (AMR) is a major public health threat, with the World Health Organization and South African Department of Health identifying the education and training of healthcare professionals on AMR and antimicrobial stewardship (ABS) in the Global Action Plan and National Strategy Framework respectively. This study describes the knowledge, attitudes and perceptions (KAP) of AMR and ABS amongst final year medical, pharmacy and nursing students at a single University in Durban, South Africa.

\section{Methods}

The study was a cross-sectional questionnaire-based survey on the KAP of final-year medical, pharmacy and nursing students at a South African University.

\section{Results}

A total of 132 questionnaires were completed (response rate $33 \%$ ), with individual response rates of $63 \%(n=63), 86 \%(n=46)$ and $9 \%$ $(n=23)$ for pharmacy, nursing and medical students respectively. The mean correct knowledge score was $88.9 \%$, with significantly lower scores seen for nursing students when compared to the other two groups. The perceived seriousness of AMR at international, national and local levels was significantly lower amongst nursing students. Only a third of all students and $45 \%$ of nursing students agreed that use of antibiotics contributes to AMR. Several nursing and medical students reported taking antibiotics for viral illnesses whilst almost a quarter of all students sampled did not consult a doctor before starting an antibiotic.

\section{Conclusion}

Several gaps in knowledge were identified, with key differences between the student groups. Attitudes and perceptions also differed substantively indicating the need for curriculum review on AMR and ABS content as suboptimal KAP may lead to negative patient outcomes.

\section{ID: 8380}

Category: Antimicrobial Resistance (SAASP)

Permission: Yes

Co-Author 1: Mojapelo, R

Co-Author 1 Institute: Department of Clinical Microbiology and Infectious Diseases, School of Pathology, Faculty of Health Sciences, University of the Witwatersrand; Centre for Tuberculosis, National Institute for Communicable Diseases

Co-Author 2: Dreyer, AW

Co-Author 2 Institute: Vermaak and Partners Pathologists, Division of Pathcare

Co-Author 3: Said, HM

Co-Author 3 Institute: Centre for Tuberculosis, National Institute for Communicable Diseases

Co-Author 4: Omar, SV

Co-Author 4 Institute: Centre for Tuberculosis, National Institute for Communicable Diseases

Co-Author 5: Joseph, L

Co-Author $\mathbf{5}$ Institute: Centre for Tuberculosis, National Institute for Communicable Diseases 
Co-Author 6: Blows, L

Co-Author 6 Institute: Centre for Tuberculosis, National Institute for Communicable Diseases

Co-Author 7: Gardee, Y

Co-Author 7 Institute: Centre for Tuberculosis, National Institute for Communicable Diseases

Co-Author 8: Ismail, F

Co-Author 8 Institute: Centre for Tuberculosis, National Institute for Communicable Diseases

Co-Author 9: Ismail, N

Co-Author 9 Institute: Centre for Tuberculosis, National Institute for Communicable Diseases; Department of Medical Microbiology, Faculty of Health Sciences, University of Pretoria

E-mail Address: richardmaredimojapelo@yahoo.com

Country: South Africa

\section{ABSTRACT TITLE: CROSS-RESISTANCE AMONG RIFAMYCINS IN MYCOBACTERIUM TUBERCULOSIS CLINICAL ISOLATES}

\section{Introduction}

High level cross-resistance among rifamycins in Mycobacterium tuberculosis isolates is commonly inferred. However, previous studies reported that the minimum inhibitory concentrations (MICs) of rifabutin (RFB) among rifampicin (RIF)-resistant M. tuberculosis carrying rpoB mutations varies depending on the mutation position.

\section{Objective}

To determine the proportion of cross-resistance among rifamycins and to assess the use GenoType MTBDRplus assay in predicting differential phenotypic susceptibility to rifamycins in M. tuberculosis isolates.

\section{Method}

A total of 300 clinical isolates of rifampicin-resistant M. tuberculosis collected between June 2015 to April 2016 during routine surveillance activities for rifampicin drug resistance in high burden districts of South Africa were included. Drug susceptibility testing for RIF, RFB and rifapentine (RFP) was performed using the BD MGIT960 instrument and the Sensititre MycoTB plate was used to determine MICs for RIF and RFB. To determine rpoB mutations, all the isolates were tested by LPA method and isolates with inconclusive results were sent for $r p o B$ gene sequencing.

\section{Results}

The proportion of cross resistance among rifamycins were: across all three (216/300; 72\%), between RIF and RFP (292/300; 98\%) and RIF and RFB (217/300; 72\%). The S531L mutation was the mostly associated with cross resistance to all rifamycins (144/153; 94\%), while the D516V mutation associated with differential susceptibility to RFB $(50 / 52 ; 96 \%)$.

\section{Conclusion}

The results show high levels of cross resistance across all rifamycins, however $28 \%$ of MDR/XDR-TB cases could potentially benefit from rifabutin as a substitute drug to the failing regimen. The use of LPA and rpoB mutations specifically S531L and D516V can be beneficial in rapidly differentiating phenotypic susceptibility to rifabutin according to this study.

\section{ID: 8381}

Category: Antimicrobial Resistance (SAASP)

Permission: Yes

Co-Author 1: Gardee, Y

Co-Author 1 Institute: Centre for Tuberculosis, National Institute for Communicable Diseases, National Health Laboratory Service; Department of Clinical Microbiology and Infectious Diseases, Faculty of Health Sciences, University of the Witwatersrand

Co-Author 2: Dreyer, AW

Co-Author 2 Institute: Department of Clinical Microbiology and Infectious Diseases, Faculty of Health Sciences, University of the Witwatersrand

Co-Author 3: Koornhof, HJ

Co-Author 3 Institute: Centre for Tuberculosis, National Institute for Communicable Diseases, National Health Laboratory Service; Department of Clinical Microbiology and Infectious Diseases, Faculty of Health Sciences, University of the Witwatersrand

Co-Author 4: Ismail, NA

Co-Author 4 Institute: Department of Medical Microbiology, Faculty of Health Science, University of Pretoria

E-mail Address: yasming@nicd.ac.za

Country: South Africa

\section{ABSTRACT TITLE: PROSPECTIVE EVALUATION OF GENOTYPE MTBDRSL VER 2.0 LINE PROBE ASSAY (LPA) FOR THE DETECTION OF SECOND-LINE MYCOBACTERIUM TUBERCULOSIS RESISTANCE IN CLINICAL SAMPLES}

\section{Introduction}

South Africa has one of the highest burdens of drug-resistant tuberculosis (DR-TB) globally, with 18734 laborator- confirmed DR-TB cases reported by WHO in 2014. Rapidity in diagnosis of DRTB would enable early and potentially appropriate and effective treatment. Phenotypic drug susceptibility testing (DST) is labour intensive with long turn-around-time (TAT) to results. Molecularbased assays designed to detect specific drug resistance mutations for M. tuberculosis, offer the advantage of faster TAT and accurate results. WHO recommended the use of Genotype ${ }^{\circledast}$ MTBDRsI VER 2.0 LPA as an initial direct DST in May 2016.

\section{Methods}

Genotype ${ }^{\oplus}$ MTBDRsI VER 2.0 LPA is designed for molecular detection of resistance-conferring mutations in genes encoding resistance to fluoroquinolones (FLQ) (gyrA and gyrB) and secondline injectable drugs (SLID) (rrs and eis). One-hundred-and-seventyeight smear positive clinical samples collected between April 2016 and November 2016 were tested using MTBDRsI VER 2.0 to assess the diagnostic performance of the assay compared with phenotypic 
DST. Sensitivity, specificity and diagnostic accuracy of MTBDRsI VER 2.0 were determined with BACTEC $^{\circledR}$ MGIT 960 DST as a reference method, using WHO 2013 recommended critical concentrations for DST.

\section{Results}

Directly comparable results between LPA and DST were available for 134 clinical samples. Sensitivity, specificity and diagnostic accuracy were calculated and are as follows respectively:

FLQ (OFX) 76.8\% (Cl: 63.6-87.0\%), 89.7\% (Cl: 80.8-95.5\%) and 84.2\%; FLQ (MFX), 79.2\% (Cl: 65.0-89.5\%), 84.9\% (Cl: 75.5-91.7\%) and 82.7\%; SLI (KAN) 78.9\% (Cl: 67.6- 87.7\%), 90.3\% (Cl: 80.1-96.4\%) and 84.2\%; SLI (AMK), 85.9\% (Cl: 75.0-93.4\%), 89.7\% (Cl: 79.9-95.8\%), and $87.9 \%$; SLI (CAP) 86.2\% (Cl: 75.3-93.5\%), 91.2\% (Cl: 81.8-96.7\%) and $88.7 \%$.

\section{Conclusion}

MTBDRsI VER 2.0 LPA performed well on clinical samples with good sensitivity and specificity for FLQ and SLID. The results are comparable with data published in the WHO 2016 Policy Guidance Document. The inclusion of this assay as an initial direct test in the SA National TB Programme will enable faster diagnosis and laboratory-based treatment options for DR-TB patients.

\section{ID: 8424}

Category: Antimicrobial Resistance (SAASP)

Permission: Yes

Co-Author 1: Sweswehan, K

Co-Author 1 Institute: Department of Medical Microbiology, National Health Laboratory Service; School of Laboratory Medicine \& Medical Science, College of Health Sciences, University of KwaZulu-Natal

Co-Author 2: Mlisana, K

Co-Author 2 Institute: Department of Medical Microbiology, National Health Laboratory Service; School of Laboratory Medicine \& Medical Science, College of Health Sciences, University of KwaZulu-Natal

E-mail Address: dr.khine85@gmail.com

Country: South Africa

\section{ABSTRACT TITLE: AMIKACIN-RESISTANT ACINETOBACTER SPECIES ARE MEDIATED BY THE APHA6 GENE AT AN ACADEMIC COMPLEX HOSPITAL IN DURBAN, KWAZULU-NATAL, SOUTH AFRICA}

\section{Introduction}

Drug resistant Acinetobacter species (Acinetobacter spp.) present serious therapeutic and infection control policy challenges globally. Although aminoglycosides have played a crucial role in the treatment of MDR Acinetobacter spp, recent reports indicated that Acinetobacter isolates are developing resistance to aminoglycosides around the globe. This study investigated the minimum inhibitory concentrations (MICs) of amikacin against Acinetobacter spp. and genes associated with its resistance. The association between amikacin resistance and clinical outcomes of patients was also determined.

\section{Method}

Clinical information from 107 patients cultured with Acinetobacter spp. was recorded during clinical ward rounds, including clinical outcomes, history of antibiotics prescribed and microbiological investigations. The 107 Acinetobacter spp. were investigated for susceptibility to antimicrobial agents in use at local hospitals. Resistant genes related to amikacin (aphA6 and aacA4 gene) were investigated by polymerase chain reaction (PCR) and sequencing. Analysis was performed on the relationship between clinical outcomes and antimicrobial resistant patterns, as well as on the amikacin MICs in resistant isolates $(n=6)$ versus their PCR results.

\section{Results}

Amikacin resistance was observed in six isolates (5.6\%). The MICs were $32(n=3)$ and $\geq 64 \mu \mathrm{g} / \mathrm{mL}(\mathrm{n}=3)$ for the amikacin resistant isolates. The aphA6 gene (797 bp) was detected in all amikacinresistant isolates. However, the aacA4 gene (489 bp) was not present in these isolates. While the majority [5/6 (83\%)] of cases were discharged, mortality rates were [1/6 (17\%)]. No underlying clinical factors were significantly associated with clinical outcome.

\section{Conclusion}

In this local setting, amikacin is commonly used with piperacillintazobactam as a second-line treatment option in general antibiotic policy. The majority, 101 (94.4\%) of the tested 107 Acinetobacter spp. isolates were susceptible to amikacin which underscores the crucial role of this antibiotic in the treatment of MDR Acinetobacter spp. All the six amikacin-resistant isolates were extensively drug resistant which is of serious concern.

The study was approved by the Ethics Committee of University of KwaZulu- Natal (Ethic approved: BE 283/12).

\section{ID: 8450}

Category: Antimicrobial Resistance (SAASP)

Permission: Yes

Co-Author 1: Human, E

Co-Author 1 Institute: Netcare Hospital

Co-Author 2: Persotam, S

Co-Author 2 Institute: Netcare Hospital

E-mail Address: erica.human@netcare.co.za

Country: South Africa

ABSTRACT TITLE: ANTIMICROBIAL SURGICAL PROPHYLAXIS FOR CAESAREAN SECTION, MAKING THE RIGHT CHOICE THROUGH COLLABORATIVE INTERVENTIONS 


\section{Introduction}

Surgical site infections (SSI) are the second most common cause of hospital acquired infections (HAI). SSIs lead to increased cost and poor patient outcomes. Appropriate antibiotic surgical prophylaxis (ASP) has been shown to reduce the occurrence of SSIs. The aim of the study was to measure compliance in terms of drug choice, dose, timing and duration of therapy for C-section.

\section{Methods}

One-thousand-and-thirty-nine patient records were reviewed prospectively over a 78-week period in the maternity ward at a private hospital in Gauteng, South Africa. Data collected from patient peri-operative documents were entered onto a standardised Microsoft Excel ${ }^{\mathrm{TM}}$ spread sheet and analysed. Problem areas were identified and addressed with individual prescribers and presented to the Antimicrobial Stewardship (AMS) Committee.

\section{Results}

Over the first 28 weeks of the study $71 \%$ ( $n=266 / 375$ ) of patients reviewed received the right choice of antibiotic, 50\% ( $n=191 / 375)$ correct dose, $7 \%(n=26 / 375)$ were compliant to the right time prior to incision (30-60 minutes) and 100\% ( $n=375 / 375)$ received duration less than 24 hours. Data analysis revealed ceftriaxone utilisation as ASP. The prescriber was approached by the pharmacist to consider changing practice to a narrower spectrum alternative. This intervention failed. The data was then presented to the hospital AMS committee in week 28 , and a decision was taken to remove ceftriaxone from all theatres. As a result, this lead to $100 \%$ ( $n=460 / 460$ ) compliance to the appropriate ASP agent measured post intervention. Under-dosing of cefazolin according to weight was also identified and compliance following feedback improved to $69 \%(n=321 / 460)$. The average time of antibiotic administration prior to incision was 14 minutes.

\section{Conclusion}

Further intervention is needed to improve compliance to correct antibiotic dose and appropriate time of administration prior to incision. The AMS committee plays an important role in a hospital to drive improvement and communicate difficulties identified to medical practitioners.

\section{ID: 8458}

Category: Antimicrobial Resistance (SAASP)

Permission: Yes

Co-Author 1: Anane, YA

Co-Author 1 Institute: 1Medical Microbiology, Department of Laboratory Medicine \& Pathology, Faculty of Health Sciences, Walter Sisulu University

\section{Co-Author 2: Apalata, $T$}

Co-Author 2 Institute: 1Medical Microbiology, Department of Laboratory Medicine \& Pathology, Faculty of Health Sciences, Walter Sisulu University

Co-Author 3: Vasaikar, $S$

Co-Author 3 Institute: 1Medical Microbiology, Department of
Laboratory Medicine \& Pathology, Faculty of Health Sciences, Walter Sisulu University

E-mail Address: anadjei@yahoo.com

Country: South Africa

ABSTRACT TITLE: SCREENING OF BLAOXA23

AND BLAIMP GENES IN MULTIDRUG RESISTANT

ACINETOBACTER BAUMANNIIISOLATED FROM CLINICAL

SPECIMENS OBTAINED FROM MTHATHA, SOUTH AFRICA

\section{Introduction}

Acinetobacter baumannii is a common opportunistic pathogen that causes major nosocomial infections in hospitals. Molecular analysis of Multidrug resistant (MDR) carbapenem-resistant genes in A. baumannii is less commonly reported from South Africa. In this study we determined the antibiotic resistance patterns and genetic mechanism of carbapenem resistance in clinical isolates of MDR A. baumannii.

\section{Method}

MDR A. baumannii isolates were collected between August 2016 and July 2017 at NHLS, after being recovered from various clinical specimens from Nelson Mandela Academic Hospital in Mthatha. The identity and antibiotic resistance of $A$. baumannii strains were determined using the VITEK ${ }^{\otimes} 2$ and confirmed by Beckman and Coulter autoSCAN-4 System. A. baumannii isolates resistance to colistin, tigecycline, imipenem, meropenem, gentamicin, amikacin, cefepime, ceftazidime, ceftriaxone, cefotaxime, ciprofloxacin, piperacillin/tazobactam, trimethoprim-sulfamethoxazole was determined. Real-time PCR was done using Roche LightCycler 2.0 to investigate carbapenemases; blaOXA-23, and blaIMP genes.

\section{Result}

Of a total of 58 multidrug resistant isolates analysed, A. baumannii exhibited high resistance rates to ciprofloxacin (93.1\%), amikacin (86.8\%), ceftazidime $(74.4 \%)$, imipenem $(71.3 .8 \%)$ and $73.4 \%$ were resistant to meropenem. The majority of the rates of antibiotic susceptibility in A. baumannii belonged to colistin and tigecycline with $99 \%$ (MICs $<2 \mu \mathrm{g} / \mathrm{ml}$ ) and $91.3 \%$ respectively. The blaOXA-23 gene was detected in $84.5 \%(n=49)$ isolates, while no blalMP was detected in any of the isolates.

\section{Conclusion}

Our study found that there is a high level of blaOXA-23 gene circulating in Mthatha. Continuous monitoring of antimicrobial resistance and strict adherence to infection prevention guidelines are essential to prevent proliferation of MDR A. baumannii.

\section{ID: 8461}

Category: Antimicrobial Resistance (SAASP)

Permission: Yes

Author: Nana, $T$

Author Institute: National Health Laboratory Service; University of the Witwatersrand 
E-mail Address: trusha.nana@mweb.co.za

Country: South Africa

\section{ABSTRACT TITLE: ANALYSIS OF THE ANTIBIOGRAM FROM AN ORTHOPAEDIC DEPARTMENT AT A TERTIARY HOSPITAL IN JOHANNESBURG}

\section{Introduction}

Changing patterns of microbial aetiology and increasing antibiotic resistance negatively impact on initiation of early effective treatment of orthopaedic infections. This may result in increased morbidity and the need for additional antibiotics and surgery. A unit-specific antibiogram assists in guiding empiric antibiotic choices.

\section{Methods}

For the period 1 July 2015 to 30 June 2017, results of all bacterial pathogens from the orthopaedic department were reviewed.

\section{Results}

There were 407 non-duplicate isolates with a similar number of Gram-positive and Gram-negative organisms.

Staphylococcus aureus made up $70 \%$ of Gram-positives. This was followed by enterococci (17\%). The remaining Gram-positives comprised of streptococci, coagulase-negative staphylococci and anaerobes.

Twenty-three percent of $S$. aureus isolates were methicillin-resistant.

Enterococcus faecalis, all ampiciliin-susceptible, was the predominant enterococcal species. There were no vancomycinresistant enterococci.

The Gram-negatives comprised of Enterobacteriaceae (63\%), nonfermenters (34\%) and anaerobes (4\%).

The predominant Enterobacteriaceae were Enterobacter species, Escherichia coli and Proteus species. Rates of susceptibility to all antibiotic groups varied by genus with Enterobacter species showing the lowest susceptibility rates and Proteus species the greatest. Overall rate of susceptibility to piperacillin-tazobactam, ceftriaxone and cefepime for these Enterobacteriaceae was 80\%, $81 \%$ and $83 \%$, respectively.

The non-fermenters comprised of equal numbers of Pseudomonas aeruginosa and Acinetobacter baumannii. Eighty-five percent of $P$. aeruginosa isolates were susceptible to ciprofloxacin and $91 \%$ to amikacin. Of the $\beta$-lactams, piperacillin-tazobactam and cefepime demonstrated the greatest activity.

Overall A. baumannii isolates showed very low rates of susceptibility to all antibiotic groups. Sixty-six percent were multidrug-resistant (MDR).

\section{Conclusion}

Tracking and reporting of resistance patterns is an important component of antibiotic stewardship. Antibiograms have many uses, including being a valuable antibiotic-prescribing tool. The antibiogram must be used in conjunction with clinical (infection type: septic arthritis, osteomyelitis, implant or surgical site infection) and epidemiological (community- or hospital-acquired infection and individual patient risk factors for MDR infection) data.

\section{ID: 8469}

Category: Antimicrobial Resistance (SAASP)

Permission: Yes

Co-Author 1: Griesel, M

Co-Author 1 Institute: Netcare Ltd

Co-Author 2: Greyling, $T$

Co-Author 2 Institute:

Co-Author 3: Leadsom, M

Co-Author 3 Institute: Netcare Ltd

E-mail Address: maletje.griesel@netcare.co.za

Country: South Africa

\section{ABSTRACT TITLE: THE EVALUATION OF THE APPROPRIATE USE OF ECHINOCANDINS FOR THE TREATMENT OF INVASIVE CANDIDIASIS IN A PRIVATE HOSPITAL SETTING}

\section{Introduction}

Invasive candidiasis (IC) is a major contributor to morbidity and mortality in non-neutropenic patients in the ICU setting. Echinocandins are the first choice of therapy for IC but their appropriate use remains a challenge. The aim of this study was to evaluate the appropriate use of echinocandins utilising the START Candida Score and the (1-3)- $\beta$-D-Glucan Assay (Fungitell assay).

\section{Methods}

This retrospective study was conducted between October 2016 and June 2017 in a private hospital. All patients who received an echinocandin, as well as their potential risk factors for IC were identified using the electronic billing database. Laboratory data were collected from the antibiotic stewardship and infection prevention database. Total parenteral nutrition (TPN), surgery, multifocal Candida colonisation and severe sepsis were coded as ' 0 ' if absent, and present ' 1 ' (severe sepsis coded ' 2 '). A Candida score of $\geq 3$ selected patients at high risk for invasive candidiasis.

\section{Results}

Thirty patients received an echinocandin. Thirteen (43\%) patients had a positive Candida score $\geq 3$ of which seven (53\%) had a positive blood culture (BC). Two patients with a negative Candida score had a positive BC. The two most prevalent Candida species isolated from blood cultures were Candida parapsilosis (55\%) and Candida albicans (33\%). A Fungitell assay was performed in four patients of whom only one was positive. The mean duration of echinocandin therapy was 12.9 days. Repeat blood cultures were only performed on two patients. 


\section{Conclusion}

More than half of the patients (57\%) received an echinocandin with a negative START Candida score. The Fungitell assay, as an adjunctive diagnostic tool, was greatly underutilised. Duration of therapy was not guided by follow-up blood cultures. The focus point for the antimicrobial stewardship committee going forward is to promote awareness and correct implementation of current guidelines regarding the diagnosis and management of IC.

\section{ID: 8478}

Category: Antimicrobial Resistance (SAASP)

Permission: Yes

Co-Author 1: Ramjathan, P

Co-Author 1 Institute: National Health Laboratory Services

Co-Author 2: Mahabeer, $\mathrm{P}$

Co-Author 2 Institute: National Health Laboratory Services

Co-Author 3: Mlisana, KP

Co-Author 3 Institute: National Health Laboratory Services

E-mail Address: praksha.ramjathan@gmail.com

Country: South Africa

\section{ABSTRACT TITLE: ANTIMICROBIAL SUSCEPTIBILITY PROFILE OF STAPHYLOCOCCUS AUREUS FROM ORTHOPAEDIC INFECTIONS}

\section{Introduction}

Staphylococcus aureus is the most commonly isolated microorganism in osteomyelitis, and more than $30 \%$ of these isolates may be methicillin resistant Staphylococcus aureus (MRSA). A study of S. aureus bacteraemia in Johannesburg showed a MRSA rate of $23 \%$. Drugs such as trimethoprim-sulfamethoxazole and rifampicin may be used as suppressive therapy in chronic osteomyelitis. Infections caused by MRSA respond poorly to empiric cloxacillin therapy. This study aimed to identify the resistance profile of $S$. aureus from orthopaedic infections in order to guide management.

\section{Methods}

The study included 84 consecutive, non-duplicate Staphylococcus aureus isolates obtained from pus swabs taken in the Orthopaedic ward at King Edward VIII Hospital. Data was extracted retrospectively from the laboratory electronic database for the period 1 January to 31 December 2016. All swabs and isolates were processed according to standard laboratory operating procedure.

\section{Results}

$77 / 84$ (93\%) of Staphylococcus aureus isolates were susceptible to cloxacillin; 76/84 (90\%) to clindamycin; 75/84 (89\%) to erythromycin; and $82 / 84(98 \%)$ to moxifloxacin. All isolates were susceptible to vancomycin, linezolid and teicoplanin. Of the seven methicillinresistant staphylococcus aureus isolates, $43 \%$ were susceptible to clindamycin, $29 \%$ susceptible to erythromycin, $14 \%$ susceptible to trimethoprim-sulfamethoxazole, $71 \%$ susceptible to moxifloxacin, $100 \%$ susceptible to vancomycin, teicoplanin, tigecycline and linezolid. Of note none of the MRSA isolates were susceptible to ciprofloxacin, rifampicin and tetracycline. The average age of patients was 34.1 years.

\section{Conclusion}

Cloxacillin is a reasonable empiric choice for the treatment of staphylococcal infections, however, drugs such as trimethoprimsulfamethoxazole and rifampicin, should not be used without susceptibility results. Specimens should be taken and therapy guided by culture results as there are serious consequences if resistance is missed and infection progresses. Vancomycin remains a good option for the treatment of MRSA.

\section{ID: 8484}

Category: Antimicrobial Resistance (SAASP)

Permission: Yes

Co-Author 1: Musyoki, A

Co-Author 1 Institute: Sefako Health Sciences University

Co-Author 2: Maluleka, C

Co-Author 2 Institute: Sefako Health Sciences University

E-mail Address: pamusyoki@gmail.com

Country: South Africa

\section{ABSTRACT TITLE: KLEBSIELLA PNEUMONIAE PREVALENCE AND ANTIMICROBIAL SUSCEPTIBILITY PROFILE: TELLING THE DR GEORGE MUKHARI ACADEMIC HOSPITAL'S STORY.}

\section{Introduction}

Both locally and globally antimicrobial resistance is a general public health concern which threatens the effective treatment of severe infections. Klebsiella pneumoniae is the most commonly encountered Gram-negative bacteria globally. It has been implicated in outbreaks with high mortality in neonatal and highcare units. This study was aimed at determining the prevalence and antimicrobial susceptibility profile of $K$. pneumoniae isolated from blood culture specimens taken at selected high-care wards of Doctor George Mukhari Academic Hospital in 2015.

\section{Methods}

Data on microbiology results of 2015 was obtained from the NHLS laboratory data information system (TrakCare) using Microsoft Excel. Blood culture results from high-care wards were filtered, double checked for repeats and categorised according to isolated microorganisms. A description of the susceptibility trends to selected antimicrobial agents commonly used against the microorganism was done. A total of 1605 blood culture results were investigated.

\section{Results}

A total of 359/1 605 (22.4\%) of the blood cultures reported isolation of $K$. pneumoniae. K. pneumoniae was found susceptibile to among others, cephalosporins at $65 \%$ and piperacillin/tazobactam at $78 \%$. The isolates were also susceptible to carbapenems; meropenem: 
100\%, imipenem: $99.7 \%$ and ertapenem: $99.7 \%$. There was a mixed level of susceptibility and resistance ratio to other agents: $64 \%$ to $36 \%$ for amoxicillin-clavulanic acid and $84 \%$ to $16 \%$ for ciprofloxacin. Out of the 359 K.pneumoniae isolated, 109 (30\%) were extended spectrum beta lactamase (ESBL) producers.

\section{Conclusion}

The level of resistance to antimicrobial agents indicated in this report affirms the need for an ongoing surveillance system that gives regular updates on antimicrobial resistance trends and to aid the update of antimicrobial policies within DGMAH.

\section{ID: 8502}

Category: Antimicrobial Resistance (SAASP)

Permission: Yes

Co-Author 1: Bornman, A

Co-Author 1 Institute: Netcare

Co-Author 2: Campbell, D

Co-Author 2 Institute: Netcare

Co-Author 3: Hutton, G

Co-Author 3 Institute: Netcare

Co-Author 4: Silberbauer, E

Co-Author 4 Institute: Ampath

Co-Author 5: Govender, N

Co-Author 5 Institute: Netcare

Co-Author 6: Botha, M

Co-Author 6 Institute: Ampath

Co-Author 7: Fingleson, L

Co-Author 7 Institute: Netcare

Co-Author 8: Conrad, N

Co-Author 8 Institute: Netcare

E-mail Address: amanda.bornman@netcare.co.za

Country: South Africa

\section{ABSTRACT TITLE: MANAGEMENT OF UNCOMPLICATED URINARY TRACT INFECTIONS IN THE EMERGENCY DEPARTMENT: A POTENTIAL OVERLOOKED CONTRIBUTOR TO ANTIBIOTIC RESISTANCE}

\section{Introduction}

Uncomplicated urinary tract infections (UTIs) are one of the most common infections in the primary healthcare setting, more commonly in adult non-pregnant women. Approximately $20 \%$ of all antibiotic prescriptions issued by general practitioners are for patients with uncomplicated UTIs and treated empirically without conducting a urine culture or susceptibility testing. This is of concern as Escherichia coli (E. Coli), which is the most prevalent organism isolated from urine cultures, has an emerging increase in resistance to quinolones.
The study aimed to assess the empiric choice of antibiotics prescribed for an uncomplicated UTI in the emergency department and to determine whether urine cultures were requested, the most prevalent organism and its sensitivity pattern.

\section{Methods}

A retrospective record review of patients that consulted in the emergency department with suspected UTI in the time period of 1 January 2014 until 29 of February 2016 was conducted. Patients admitted to the hospital, complicated UTIs and recurrent UTIs were excluded.

\section{Results}

Three hundred records were reviewed. The majority of the study population was female (88\%), with a mean age of 33.3 years. Urine cultures were requested in only $56 \%(n=168)$ of the patients presenting with signs and symptoms of an uncomplicated UTI. Cultures were positive in $77 \%(n=131)$ of cases and E.Coli was the most prevalent organism isolated $(58.82 \%, \mathrm{n}=100)$, with Klebsiella pneumonia accounting for $4.71 \%(n=8)$ of the isolates.

Ciprofloxacin was prescribed in the majority of cases (37.9\%), followed by amoxicillin/clavulanate (29\%); $23.3 \%(n=70)$ of patients received two or more antibiotics, either in class of antibiotic or route of administration, with $n=54$ of these patients receiving intravenous antibiotics.

\section{Conclusion}

Antibiotic stewardship principles need to be applied in the emergency department, with special emphasis on double antimicrobial cover as well as establishing an ethos to perform cultures prior to antibiotic prescribing.

\section{ID: 8545}

Category: Antimicrobial Resistance (SAASP)

Permission: Yes

Co-Author 1: Omar, SV

Co-Author 1 Institute: Centre for Tuberculosis, Supranational TB Reference Laboratory, National Institute for Communicable Diseases, National Health Laboratory Service

Co-Author 2: Ismail, F

Co-Author 2 Institute: Centre for Tuberculosis, Supranational TB Reference Laboratory, National Institute for Communicable Diseases, National Health Laboratory Service/Department of Medical Microbiology

Co-Author 3: Joseph, L

Co-Author 3 Institute: Centre for Tuberculosis, Supranational TB Reference Laboratory, National Institute for Communicable Diseases, National Health Laboratory Service

Co-Author 4: Mvusi, L

Co-Author 4 Institute: TB Control \& Management Cluster, National Department of Health, South Africa

Co-Author 5: Ismail, NA 
Co-Author 5 Institute: Centre for Tuberculosis, Supranational TB Reference Laboratory, National Institute for Communicable Diseases, National Health Laboratory Service/Department of Medical Microbiology

E-mail Address: shaheedvo@nicd.ac.za

Country: South Africa

\section{ABSTRACT TITLE: PREVALENCE OF THE RIFAMPICIN RESISTANT DETERMINANT AT CODON 491 IN SOUTH AFRICA, UNDETECTED BY COMMERCIAL PHENOTYPIC AND GENOTYPIC METHODS}

\section{Introduction}

The commercial WHO-endorsed phenotypic and molecular assays are incapable of detecting rifampicin resistance in Mycobacterium tuberculosis clinical strains harbouring the resistance conferring lle491Phe mutation found outside the Rifampicin ResistanceDetermining Region (RRDR). Of concern to South Africa, is a publication showing that $30 \%$ of MDR-TB cases in Swaziland, partially nestled within South Africa, harbour this mutation. We aimed to determine the prevalence of this mutation by retrospective analysis of Whole Genome Sequenced isolates representing two provinces, in close proximity to Swaziland, from the 2014 National TB Drug Resistance Survey (DRS).

\section{Methods}

Survey isolates from two provinces were sequenced using NextGeneration Whole Genome Sequencing methodology. The provinces included Gauteng, the financial hub which attracts foreign migrants, and KwaZulu-Natal, the province with the highest burden of TB disease bordering Swaziland's eastern perimeter. Isolates were further subjected to drug susceptibility testing on the MGIT960 to first and second-line anti-tuberculosis drugs.

\section{Results}

Of the 1535 genomes sequenced from the TB DRS, 1 (0.07\%) isolate (Gauteng) harboured the lle491Phe as a minor variant $(2 \%$ frequency). This isolate was fully susceptible to both first- and second-line drugs. The crude unadjusted prevalence of resistance conferring mutations within the RRDR occurred at 5.15\% (79/1535). Inclusion of the lle491Phe mutation adjusts the prevalence to $5.21 \%$ (80/1535).

\section{Conclusion}

Despite the prevalence of this mutation at high rates in Swaziland, the prevalence was only found to be $0.07 \%$ in the two provinces or $0.12 \%$ when considering Gauteng only. Due to the clinical implications of not detecting this mutation, surveillance activities have been enhanced to improve detection of this mutation. It would be impractical to screen all isolates based on the total number of TB diagnosed annually in South Africa, therefore, clinicians should bear in mind the occurrence of this mutation in cases with poor clinical outcomes despite adherence to therapy.

\section{ID: 8635}

Category: Antimicrobial Resistance (SAASP)

Permission: Yes

Co-Author 1: Mthiyane, HR

Co-Author 1 Institute: Witwatersrand University

Co-Author 2: Danaviah, $S$

Co-Author 2 Institute: Africa Health Research Institute (AHRI);

Co-Author 3: de Oliveira, $T$

Co-Author 3 Institute: University ok KwaZulu Natal (Medical school)

Co-Author 4: Viljoen, J

Co-Author 4 Institute: Witwatersrand University

E-mail Address: hloni.mthiyane@wits.ac.za

Country: South Africa

\section{ABSTRACT TITLE: PREVALENCE OF MINORITY HIV- 1 DRUG RESISTANT MUTATIONS IN CHILDREN WITH VIROLOGIC FAILURE IN A RURAL KWAZULU-NATAL COHORT}

\section{Introduction}

Missed minority drug resistance mutations (DRMs) may pave a way to therapy failure in a short period of time. Therefore, assays have been developed including next generation sequencing (NGS) that are able to identify a larger proportion of mutations including those bearing minority DRMs within a patient's viral population. The aims of this study were to (1) describe the prevalence of minority HIV DRMs in paediatric population with virologic failure in a rural KwaZulu-Natal (KZN) paediatric cohort using NGS technology, and (2) to compare the genotypes generated using Sanger sequencing with NGS.

\section{Methods}

Thirty-four patients were genotyped using Sanger sequencing and NGS. A $1.3 \mathrm{~kb}$ region of the Pol gene was genotyped using Sanger sequencing. The whole $9.7 \mathrm{~kb}$ HIV genome was sequenced using NGS. All electropherograms were analysed using the Geneious V8.0.5 software system for the presence of DRMs including minority variants. Sequences were assembled against an HIV-1 subtype $C$ reference sequence from South Africa. For NGS a reference sequence was annotated with known HIV resistance mutations within the protease and RT genes.

\section{Results}

NGS was able to detect minoritydrug resistance mutations in eleven (32.3\%) samples which were missed by Sanger sequencing. NGS also detected an additional three (8.8\%) specimens that harboured DRMs but were found to be susceptible by Sanger sequencing.

\section{Conclusion}

The presence of minority DRMs among children is likely to obstruct the use of ART and consequently predispose patients to therapy failure.We noted that children on PI-based regimen, while at a lower prevalence, still harboured DRMs that remained undetected 
by conventional Sanger sequencing. Finally, this study emphasised the need to apply more sensitive assays to accurately distinguish patients failing due to the emergence of minority DRMs from those that are non-adherent in order to maximise the efficacy of the limited range of anti-retroviral drugs currently in use in South Africa.

\section{ID: 8237}

Category: Infectious Diseases (IDSSA)

Permission: Yes

Co-Author 1: Nkwinika, VV

Co-Author 1 Institute: Sefako Makgatho Health Sciences University

Co-Author 2: Lebelo, RL

Co-Author 2 Institute: Sefako Makgatho Health Sciences University E-mail Address: vnkwinika@gmail.com

Country: South Africa

\section{ABSTRACT TITLE: PREVALENCE OF HUMAN PAPILLOMAVIRUS INFECTIONS IN ANORECTAL CANCERS AT THE DR GEORGE MUKHARI ACADEMIC HOSPITAL FROM 2005 TO 2012}

\section{Introduction}

Human papillomavirus (HPV) accounts for $94 \%$ of anorectal cancer cases globally. The study aimed at investigating the prevalence of HPV-genotypes in anorectal cancers and reports on the prevalence of HPV-infections and HPV-genotypes in different histological sites of anorectal cancers.

\section{Methods}

HPV-DNA from 148 formalin-fixed paraffin-embedded (FFPE) tissue samples was extracted using NucliSENS ${ }^{\circledR}$ easyMAG ${ }^{\circledR}$ following manufacturer's protocol with an off-board pre-lysis step. All DNA extracts were screened for HPV-DNA with a nested-PCR assay (MY/ GP primer-set) targeting the L1-gene. Genotyping was carried out using Linear Array HPV genotyping assay. All HPV-positive samples were tested for HPV-E6/E7 mRNA expression using APTIMA HPV Assay. Descriptive data analysis on Epi Info version 7.1.5 was then used to calculate frequencies of categorical data.

\section{Results}

Overall HPV prevalence of 23/148 (16\%) was found. Of these, the majority $14 / 23$ (61\%) were males compared to $9 / 23$ (35\%) females. Only eight HPV genotypes (HPV-6, 11, 16, 18, 33, 82, 84, and 66) were detected. Non-oncogenic HPV-6 was predominant in 8/23 (35\%). Adenocarcinoma of the rectum region was frequently observed in 133/148 (90\%). Anorectal cancer was more prevalent in patients older than age 50 (mean age 54.4; std. Dev 17.9). There was only $1 / 23(4 \%)$ of the samples (HPV-33) that expressed HPV-E6/E7 mRNA.

\section{Conclusion}

Non-oncogenic HPV-types observed in the majority of adenocarcinoma of the rectum may not necessarily imply any aetiological role in anorectal cancer development. Since the majority of the advanced-aged population present with higher anorectal cancer prevalence, caution should be taken in youngeraged population to prevent cancer development at a later lifestage, especially in males.

\section{ID: 8340}

Category: Infectious Diseases (IDSSA)

Permission: Yes

Co-Author 1: Patharoo, V

Co-Author 1 Institute: National Health Laboratory Services, National Institute of Communicable Diseases

E-mail Address: venessap@nicd.ac.za

Country: South Africa

\section{ABSTRACT TITLE: ARBOVIRUS LABORATORY INVESTIGATION OF A CLUSTER OF CASES PRESENTING WITH FEVER AND RASH IN JOHANNESBURG, 2017}

\section{Introduction}

An investigation into generalised maculopapular rash reported by dermatologists in a cluster of cases in Johannesburg, was initiated by the Outbreak Response Unit of the NICD at the end of January 2017. Arthropod-borne virus (arbovirus) infection was considered as a possible differential diagnosis based on clinical presentation of the patients and the season.

\section{Methods}

Major endemic arboviruses were included in the laboratory investigation at the Centre for Emerging and Zoonotic Diseases: West Nile, Sindbis, Chikungunya and Rift Valley fever. Differential laboratory diagnosis included a battery of assays targeting specific antibodies (haemagglutination inhibition assay, ELISA, virus neutralisation test), viral RNA (PCR, sequencing) and infectious virus by attempted isolation in suckling mice or in tissue culture.

\section{Results}

A total of 47 suspected cases were referred to the NICD for testing. $\operatorname{lgM}$ antibodies against Sindbis virus were detected in 27 serum samples, indicative of recent Sindbis virus infection. In addition, recent infection was confirmed in 11 patients by the testing of paired sera, taken two weeks apart to detect a significant rise in antibody titre. Sindbis virus RNA could not be detected in any of the patients, presumably due to blood specimens taken post viremic stage of infection - Sindbis virus is causing typically transient and low viraemia.

\section{Conclusion}

Sindbis virus is a mosquito-borne arbovirus which is maintained in a mosquito-bird transmission cycle. Sindbis virus infection in humans has a short incubation period of less than seven days and is usually a mild and self-limiting febrile illness, but chronic joint manifestations may occur in some cases. The cluster of cases was rare compared 
to the number of Sindbis cases usually reported in the past. This is, however likely a reflection of the enhanced passive surveillance and the increased awareness of rash cases reported by dermatologists from January 2017.

\section{ID: 8581}

Category: Infectious Diseases (IDSSA)

Permission: Yes

Co-Author 1: Mokwena, B

Co-Author 1 Institute: National Health Laboratory Service, Dr George Mukhari Tertiary Laboratory

Co-Author 2: Makhado, N

Co-Author 2 Institute: National Health Laboratory Service, Sefako Health Sciences University

Co-Author 3: Maluleka, C

Co-Author 3 Institute: National Health Laboratory Service, Sefako Health Sciences University

Co-Author 4: Nchabeleng, $M$

Co-Author 4 Institute: National Health Laboratory Service, Sefako Health Sciences University

E-mail Address: nmakhado@yahoo.com

Country: South Africa

\section{ABSTRACT TITLE: EVALUATION OF COBAS E601 SYPHILIS ASSAY FOR THE DETECTION OF SYPHILIS}

\section{Introduction}

Treponema pallidum is a causative agent of syphilis infections, which can lead to severe complications if not diagnosed and treated rapidly. The diagnosis as well as screening of syphilis often require assays with high specificity and sensitivity. The Cobas E601 assay is an automated immunoassay which detects antibodies against T. pallidum. The test is intended as an aid in the diagnosis of syphilis infection with total assay duration of about 20 minutes. This study was done to evaluate the diagnostic performance of E601 Syphilis cobas assay for the detection of syphilis by comparing with Serodia TPPA assay.

\section{Methods}

A total of 67 human residual serum samples that were sent for routine laboratory screening for syphilis were collected, Performance of Cobas E601 Syphilis assay was evaluated by determining its sensitivity and specificity compared to Serodia TPPA assay.

\section{Results}

Of 67 samples tested, 12/67 (18\%) were found to be reactive using the Cobas E601 Syphilis assay and 55/67 (82.0\%) were found to be nonreactive. The overall sensitivity and specificity of the Cobas E601 Syphilis assay as compared to the Serodia TPPA assay was $98.2 \%$ and $100 \%$, respectively.

\section{Conclusion}

Cobas E601 Syphilis assay demonstrated good diagnostic performance when used to detect syphilis from serum or plasma samples. It can be used as an alternative to Serodia TPPA assay. The assay also has an advantage of less hands-on time and shorter TAT.

\section{ID: 8464}

Category: Sexually Transmitted Diseases (STDSSA)

Permission: Yes

Co-Author 1: Mabaso, N

Co-Author 1 Institute: University of Kwazulu-Natal

Co-Author 2: Sturm, A

Co-Author 2 Institute: University of Kwazulu-Natal

E-mail Address: ncamo.mabaso@gmail.com

Country: South Africa

\section{ABSTRACT TITLE: THE EFFECT OF ANTIMICROBIALS USED FOR GENITAL DISCHARGE DISEASE ON TRICHOMONAS VAGINALIS}

\section{Introduction}

Trichomoniasis is the most common sexually transmitted infection caused by the protozoan, Trichomonas vaginalis. T. vaginalis infection is often asymptomatic. This infection causes vaginal discharge in women and urethritis in men. It has been reported that trichomoniasis is associated with serious health complications and it increases the risk of HIV acquisition and transmission. Metronidazole has been the standard treatment for trichomoniasis. Multiple cases of metronidazole-resistance have been reported since 1962. Syndromic management of STIs is used to treat multiple infections simultaneously based on the signs and symptom with which the patient presents. In South Africa, the vaginal discharge syndrome is managed with ceftriaxone, azithromycin and metronidazole.

\section{Methods}

Ten $T$. vaginalis isolates were tested. Each isolate was tested against six combinations of two antimicrobials by the checkerboard method, four combinations of three antimicrobials and two combinations of four antimicrobials. The results obtained from the checkerboard of two antimicrobials were used to design the experiments for three and four antimicrobials combinations.

\section{Results}

The MICs for metronidazole ranged between $0.25-1 \mu \mathrm{g} / \mathrm{ml}$ and for doxycycline, they ranged between 64-128 $\mu \mathrm{g} / \mathrm{ml}$. Ceftriaxone and fluconazole showed no antitrichomonal activity. All combinations tested had an indifferent effect. However, in the combination of metronidazole and doxycycline a decrease in the MICs for these antibiotics was observed.

\section{Conclusion}

Combinations of metronidazole and antimicrobials used in syndromic management including fluconazole has no effect against T. vaginalis. The decrease in MICs of metronidazole and doxycycline in their combination suggests that these two antimicrobials have 
some degree of synergism although it was interpreted as indifferent by the FIC Indices. Therefore, it is essential to further investigate this combination with a larger sample size.

\section{ID: 8551}

Category: Sexually Transmitted Diseases (STDSSA)

Permission: Yes

Co-Author 1: Gosnell, B

Co-Author 1 Institute: Nelson R Mandela School of Medicine, University of KwaZulu-Natal

Co-Author 2: Moosa, MS

Co-Author 2 Institute: Nelson R Mandela School of Medicine, University of KwaZulu-Natal

E-mail Address: Bkarges@hotmail.com

Country: South Africa

\section{ABSTRACT TITLE: CHRONIC HSV-2 ULCER IN HIV+ WOMAN FROM GENITAL AREA TO FINGER}

\section{Introduction}

Herpes simplex virus (HSV) infection is common in HIV-positive populations and can cause debilitating chronic ulcers. A short course of acyclovir is part of the national treatment guidelines for genital ulcer disease.

\section{Case report}

A female HIV positive patient presented to King Edward VIII hospital, Durban, in 2007 with a six-month history of non-healing peri-anal lesion, histologically HSV, which was excised. She presented in 2008 with a recurrence of a similar lesion on her right labia minora, HSV-2 positive on PCR and histology. Her antiretrovirals were changed to a PI based regimen due to failure. High dose IV acyclovir for two weeks, followed by three weeks of IV foscarnet failed to shrink the exudating lesion, which had grown to $>20 \mathrm{~cm}^{2}$, with a new satellite lesion developing on right perineum. Treatment with topical 1\% cidovovir cream reduced the amount of exudation and led to re-epithelialisation of a small area. Unfortunately, the donated stock ran out and topical cidofovir had to be stopped. The lesion continued to grow over the next weeks. Off-label use of topical imiquimod lead to marked improvement within two weeks and near complete healing over three months.

Unfortunately, due to shortage of stock on recurrence, imiquimod could not immediately be resumed and her lesion grew larger. When restarting topical imiquimod, she managed to auto-inoculate her left thumb with herpetic whitlow. Both lesions were scraped and growth of HSV 2 obtained in cell culture. Both isolates were extremely acyclovir resistant. Over the course of several months both lesions responded to imiquimod.

\section{Discussion}

Failure of syndromic management of genital ulcers should prompt referral to a competent tertiary centre. Chronic HSV ulcers in the HIV infected can be challenging to heal. This case along with more than five others treated in the King Edward VIII hospital Infectious diseases unit, illustrate the success with topical imiquimod.

\section{ID: 8554}

Category: Sexually Transmitted Diseases (STDSSA)

Permission: Yes

Co-Author 1: Lebelo, RL

Co-Author 1 Institute: Sefako Makgatho Health Sciences University

Co-Author 2: Selabe, G

Co-Author 2 Institute: Sefako Makgatho Health Sciences University Co-Author 3: Tiiti, T

Co-Author 3 Institute: Sefako Makgatho Health Sciences University Co-Author 4: Mashishi, $T$

Co-Author 4 Institute: Sefako Makgatho Health Sciences University Co-Author 5: Nkwinika, V

Co-Author 5 Institute: Sefako Makgatho Health Sciences University

E-mail Address: ramslisbeth@yahoo.com

Country: South Africa

\section{ABSTRACT TITLE: HPV MRNA EXPRESSION AND HPV DNA PREVALENCE IN WOMEN ATTENDING GYNAECOLOGY CLINICS AT DGMAH, PRETORIA}

\section{Introduction}

Persistent high-risk HPV infection has been established as a strong risk factor for progression of cervical precursor lesions to cervical cancer. Cervical cancer in South Africa is rated the second leading cause of death in women and up to $70 \%$ of cervical cancer cases are caused by HPV 16 and 18. In South African women it is reported that HPV DNA is present in $63.9 \%$ of cervical cancer cases. HPV DNA detection does not necessarily mean the patients will develop cervical lesions, it is then important to use makers that will assist in management of patients and screening.

\section{Methods}

This is an ongoing cross-sectional study and thus far 280 samples have been analysed. HPV testing was performed using Abbott m2000rt that detects $14 \mathrm{HR}$ HPV types and partial genotyping for HPV 16 and HPV 18. HPV E6/E7 mRNA was detected using Aptima HPV HR assay on panther system detecting 14 HR HPV types and this test was performed in only HPV DNA samples. Women 18 years and older are enrolled at gynecology clinics at DGMAH. Liquidbased cytology samples are collected by a nurse / doctor.

\section{Results}

Of the 280 samples, $51.1 \%$ had detected HPV DNA, of these HPV 16 was positive in $9.6 \%$ while HPV 18 was detected in $4.6 \%$. Other HR types were present in 95.45 of the samples. Of those positive for HPV type 16, HPV mRNA showed expression in 88.9\%. For HPV 18 positive samples $76.9 \%$ had detectable mRNA. A high percentage (76.7\%) of those positive for other HR HPV types also showed 
expression of HPV mRNA. Overall HPV mRNA was present in 69.9\% of all the HPV DNA samples.

\section{Conclusion}

Almost half the patients attending gynaecology clinics at DGMAH harbour HPV. HPV mRNA expression was detected in a high number of these samples, these may indicate an increased risk of patients to develop pre-cancerous cervical lesions.

\section{ID: 8564}

Category: Sexually Transmitted Diseases (STDSSA)

Permission: Yes

Co-Author 1: Garrett, N

Co-Author 1 Institute: Centre for the AIDS Programme of Research in South Africa

Co-Author 2: Yende-Zuma, N

Co-Author 2 Institute:

Co-Author 3: Ngcapu, S Centre for the AIDS Programme of Research in South Africa

Co-Author 3 Institute: Centre for the AIDS Programme of Research in South Africa

Co-Author 4: Liebenberg, L

Co-Author 4 Institute: Centre for the AIDS Programme of Research in South Africa

E-mail Address: andilemtshali4@gmail.com

Country: South Africa

\section{ABSTRACT TITLE: THE IMPACT OF STI CLEARANCE ON GENITAL INFLAMMATION IN HIV-INFECTED WOMEN}

\section{Introduction}

Sexually transmitted infections (STIS) and bacterial vaginosis (BV) have been linked to genital inflammation in women. However, the impact of STI clearance on genital inflammation is poorly understood. This study aimed to investigate this relationship in HIV infected women.

\section{Methods}

A total of 27 HIV-infected women, 18 years and older, were included in the study. Participants were enrolled in the CAPRISA 002 Acute Infection study from rural Vulindlela and urban eThekwini KwaZulu-Natal sites. All participants were infected with STI and subsequently cleared infections by the next study visit (6-12 months later). Genital concentrations of 27 cytokines were assessed in cytobrush supernatants collected at both visits. Genital inflammation was defined as having $\geq 4$ of 8 pro-inflammatory or chemotactic cytokines above the $75^{\text {th }}$ percentile. Bacterial vaginosis was determined by Nugent scoring. Blood CD4 counts, HIV loads, contraception use, and behavioural data were collected.

\section{Results}

Five of the $7 \mathrm{STI}+$ women (70\%) with baseline genital inflammation lost their inflammation status on STI clearance. However, due to inflammation maintained or acquired despite STI clearance, there was no significant difference in the frequency of inflamed participants in the STI-infected (22\%) and cleared (21\%) groups $(p=0.2500)$. Even after adjusting for CD4 count and viral load, clearing STI did not impact the overall frequency of inflamed HIVinfected participants.

\section{Conclusion}

These data suggest that STI-associated inflammation may be eased on STI clearance. However, further studies are warranted to identify other factors driving genital inflammation in the absence of STIs.

\section{ID: 8051}

Category: Antimicrobial Resistance (SAASP)

Permission: Yes

Co-Author 1: Authors: Black, J

Co-Author 1 Institute: Department of Biochemistry and Microbiology, Nelson Mandela Metropolitan University

Co-Author 2: Annear, D

Co-Author 2 Institute: Department of Biochemistry and Microbiology, Nelson Mandela Metropolitan University

Co-Author 3: Govender S

Co-Author 3 Institute: Department of Infectious Diseases, Livingstone Hospital; Department of Medicine, University of Cape Town

E-mail Address: sharlene.govender@nmmu.ac.za

Country: South Africa

\section{ABSTRACT TITLE MULTILOCUS SEQUENCE TYPING OF CARBAPENEM-RESISTANT PSEUDOMONAS AERUGINOSA ISOLATES FROM PATIENTS PRESENTING AT PORT ELIZABETH HOSPITALS, SOUTH AFRICA}

\section{Background}

Pseudomonas aeruginosa is an important nosocomial pathogen that exhibits multiple drug resistance with increasing frequency, especially to carbapenems making patient treatment difficult. Carbapenem-resistance may be caused by porin gene mutations, active drug efflux, and carbapenemase production. This study evaluated the incidence of genes responsible for carbapenemase production in carbapenem-resistant Pseudomonas aeruginosa and assessed the genetic relatedness of the isolates by multi-locus sequence typing (MLST).

\section{Materials and Methods}

Identification and antimicrobial susceptibility testing of $P$. aeruginosa isolates ( $\mathrm{n}=234$ ) by the VITEK 2 system detected 81 carbapenemresistant $P$. aeruginosa isolates. PCR and DNA sequencing were used to screen isolates for three metallo- $\beta$-lactamase encoding genes. MLST included amplification of seven housekeeping genes and sequence type alignment using the online $P$. aeruginosa MLST database. 


\section{Results}

Only the blaVIM-2 gene was detected in 15 of the 81 carbapenemresistant isolates. MLST indicated six different novel sequence types among the blaVIM-2 positive $P$. aeruginosa isolates with the majority of the isolates $(9 / 15)$ containing identical allelic profiles of the sequence type allocated ST1 (provisionally assigned sequence type, awaiting addition of new sequence types to PubMLST database). Five of these ST1 isolates were from patients and an environmental sample in the same hospital ward suggesting an environmental reservoir. Carbapenem-resistance in the blaVIM-2 negative isolates may be due to other mechanisms.

\section{Conclusion}

The incidence of genes responsible for carbapenemase production in carbapenem-resistant Pseudomonas aeruginosa and genetic relatedness of these isolates in public healthcare facilities within the Port Elizabeth area is of concern and requires further investigation.

\section{ID: 8275}

Category: Antimicrobial Resistance (SAASP)

Permission: Yes

Co-Author 1: Pandeya, D

Co-Author 1 Institute: Comanche County Memorial Hospital, Lawton, Oklahoma

Co-Author 2: Strickland, D

Co-Author 2 Institute: Fusion Medical Staffing

Co-Author 3: Grammer, A

Co-Author 3 Institute: The University of Texas Medical Branch, Galveston Texas

E-mail Address: drona.pandeya@gmail.com

Country: United States of America

\section{ABSTRACT TITLE: EVALUATION OF RESISTANCE PATTERN OF VARIOUS CANDIDA SPECIES}

\section{Introduction}

Because of the increased incidence of resistant strains of Candida infections, to the antifungal agents, it is imperative to know the effective antifungal susceptibility pattern in a timely manner for the treatment of patients. The purpose of this study was to determine antifungal resistant pattern among different Candida species so that appropriate antifungals could be administered to the patients with Candida infections.

\section{Methods}

A total of thirty-five individual isolates of various Candida species isolated from blood and body fluid specimens of patients admitted to the University of Texas Medical Branch, Texas from November 1, 2012 to April 30, 2013 in were included in this study. All the isolates were subjected for Epsilometer Test (Etest). The minimum Inhibitory Concentration (MIC) end points were determined for four antifungal agents such as fluconazole, itraconazole, voriconazole, and micafungin.

\section{Results}

Out of 35 isolates, C. albicans $(n=6), C$. dublinensis $(n=5), C$. tropicalis $(\mathrm{n}=6)$, C. glabrata $(\mathrm{n}=7)$, C. parapsilosis $(\mathrm{n}=6), \mathrm{C}$. krusei $(\mathrm{n}=3)$ and C. lusitaniae $(n=2), 67 \%(n=4)$ of $C$. parapsiplopsis were resistant to micafungin. All the isolates $(100 \%)(n=3)$ of $C$. krusei were resistant to fluconazole followed by $20 \%(n=1)$ of $C$. dublinensis. We also found that $71 \%(n=5)$ of $C$. glabrata were resistant to itraconazole followed by $17 \%(n=1)$ of the C. albicans, $20 \%(n=1)$ of the C. dublinensis and $33 \%(n=1) C$. krusei. None of the isolates were resistant to voriconazole.

\section{Conclusion}

Distinct antifungal resistance patterns were noted amongst different Candida species. Candida species other than albicans showed more resistance against the antifungal agents. Voriconazole was most effective antifungal against all the Candida species.

\section{ID: 8398}

Category: Antimicrobial Resistance (SAASP)

Permission: Yes

Co-Author 1: Skosana, LB

Co-Author 1 Institute: Department of Medical Microbiology, Tshwane Academic Division, National Health Laboratory Services; Department of Medical Microbiology, Faculty of Health Sciences, University of Pretoria

Co-Author 2: Bosch, A

Co-Author 2 Institute: Department of Medical Microbiology, Tshwane Academic Division, National Health Laboratory Services; Department of Medical Microbiology, Faculty of Health Sciences, University of Pretoria

Co-Author 3: Amutenya, VM

Co-Author 3 Institute: Department of Medical Microbiology, Tshwane Academic Division, National Health Laboratory Services; Department of Medical Microbiology, Faculty of Health Sciences, University of Pretoria

Co-Author 4: Ismail, F

Co-Author 4 Institute: Department of Medical Microbiology, Tshwane Academic Division, National Health Laboratory Services; Department of Medical Microbiology, Faculty of Health Sciences, University of Pretoria; Centre for Tuberculosis, National Institute of Communicable Diseases

Co-Author 5: Mbelle, NM

Co-Author 5 Institute: Department of Medical Microbiology, Tshwane Academic Division, National Health Laboratory Services; Department of Medical Microbiology, Faculty of Health Sciences, University of Pretoria

\section{Co-Author 6: Kock MM}

Co-Author 6 Institute: Department of Medical Microbiology, Tshwane Academic Division, National Health Laboratory Services; Department of Medical Microbiology, Faculty of Health Sciences, University of Pretoria

Co-Author 7: Strydom, K

Co-Author 7 Institute: Department of Medical Microbiology, Tshwane Academic Division, National Health Laboratory Services; Department of Medical Microbiology, Faculty of Health Sciences, 
University of Pretoria

E-mail Address: busiskosana@yahoo.com

Country: South Africa

ABSTRACT TITLE: COMPARISON OF THE MODIFIED CARBAPENEM INACTIVATION METHOD (MCIM) WITH THE RAPIDEC ${ }^{\oplus}$ CARBANP TEST (BIOMERIEUX, FRANCE) FOR THE PHENOTYPIC CONFIRMATION OF CARBAPENEMASE PRODUCTION IN ENTEROBACTERIACEAE

\section{Introduction}

The rapid laboratory detection of carbapenemase producing Enterobacteriaceae (CPE) is of utmost importance to guide both treatment and infection control interventions. Various phenotypic confirmatory tests have been developed to aid this. In this study we have compared the performance of the modified carbapenem inactivation method ( $\mathrm{mCIM}$ ) and the Rapidec ${ }^{\circledast}$ CarbaNP test (bioMérieux, France).

\section{Methods}

A total of 68 stored Enterobacteriaceae were evaluated using the $\mathrm{mCIM}$ as outlined in document M100-S27 of the Clinical and Laboratory Standards Institute (CLSI) guidelines as well as the Rapidec $^{\circledast}$ CarbaNP test according to manufacturer instructions. These 68 isolates were stratified as follows: 39 genotypically confirmed CPE (5 NDM, 8 OXA-48-like, 8 KPC, 10 VIM and 8 IMP) and 29 ESBL and AmpC producing Enterobacteriaceae. The sensitivity and specificity of the two tests under evaluation were determined.

\section{Results}

The sensitivity and specificity of the MCIM test was $97.4 \%$ and 100\% respectively. The OXA 163 positive isolate was not detected by the mCIM method. The sensitivity and specificity of the Rapidec ${ }^{\oplus}$ CarbaNP test was $84.6 \%$ and $100 \%$ respectively. Five of the eight OXA-48-like positive isolates (OXA-181, OXA-232, OXA-370, OXA244, and OXA-163) were not detected by the Rapidec ${ }^{\circledR}$ CarbaNP test. The Rapidec ${ }^{\circledR}$ CarbaNP test also failed to detect the VIM 23 positive isolate.

\section{Conclusion}

The $\mathrm{mCIM}$ test performed satisfactorily for the phenotypic confirmation of carbapenemase production. The Rapidec ${ }^{\circledast}$ CarbaNP test had poor sensitivity (84.6\%) for the detection of carbapenemase production, in addition the sensitivity for the detection of OXA-48like producing Enterobacteriaceae was $37.5 \%$, making it unsuitable for routine use in settings with high prevalence of OXA-48 like producers. The specificity of both tests was $100 \%$. The low cost and ease of performance of the $\mathrm{mCIM}$ test combined with the excellent sensitivity and specificity makes it ideal for the detection of CPE in our resource-constrained setting.

\section{ID: 8421}

Category: Antimicrobial Resistance (SAASP)

Permission: Yes
Co-Author 1: Bashar, MA

Co-Author 1 Institute: University of the Witwatersrand

Co-Author 2: Miot, J

Co-Author 2 Institute: University of the Witwatersrand

Co-Author 3: Shoul, E

Co-Author 3 Institute: University of the Witwatersrand

Co-Author 4: Robyn, VZ

Co-Author 4 Institute: University of the Witwatersrand

E-mail Address: basharaugie14@gmail.com

Country: South Africa

\section{ABSTRACT TITLE: IMPROVING ANTIBIOTIC UTILISATION IN SURGERY WARDS}

\section{Introduction}

The discovery of antibiotics has transformed modern medicine by playing a critical role in the management of infectious diseases. However, rapid development of resistance by bacteria is gradually affecting this initial success. Antimicrobial stewardship (AMS) programmes along with infection and prevention control measures have been shown to reduce the burden of antimicrobial resistance in hospitals. Evidence of the impact of AMS programmes in a surgical setting is limited.

\section{Methods}

An AMS ward round was implemented in two surgical wards of a tertiary South African hospital with two stages - a retrospective baseline and a prospective intervention stage. The appropriateness of antibiotic utilisation was determined using a guideline developed by Gyssens and colleagues. Additionally, the volume of antibiotic consumption was determined by DDDs/ 1000 patients' bed-days.

\section{Results}

In both stages of the study amoxicillin/clavulanic acid was the most frequently used agent. A significant reduction in the duration of antibiotic therapy for two days and more was observed from 4.74 \pm 4.58 days in the baseline stage compared to $3.96 \pm 2.04$ days in the intervention phase $(p=0.01)$. There was a significant reduction in the volume of antibiotic consumption from a total of $739.30 \mathrm{DDDs} /$ 1000 patient days in the baseline stage to 564.93 DDDs/ 1000 patient days in the intervention period ( $p=0.038$ ). There was a significant reduction of inappropriate antibiotic utilisation from $35 \%$ in the baseline stage to $26 \%$ in the intervention stage $(p=0.006)$. This was associated with a reduction in the average antibiotic cost in one of the study wards. Gram-negative bacteria were the most prevalent pathogens in both stages of the study.

\section{Conclusion}

Implementation of an AMS ward round showed an improvement in the appropriateness of antibiotic utilisation, along with reduction in consumption and cost of antibiotics in an in-patient surgical setting. 


\section{ID: 8448}

Category: Antimicrobial Resistance (SAASP)

Permission: Yes

Co-Author 1: Kingsburgh, $C$

Co-Author 1 Institute: University of Pretoria

Co-Author 2: Kock, M

Co-Author 2 Institute: University of Pretoria

Co-Author 3: Mbelle, N

Co-Author 3 Institute: University of Pretoria

Co-Author 4: Ismail, F

Co-Author 4 Institute: University of Pretoria

E-mail Address: chanelmaree@gmail.com

Country: South Africa

\section{ABSTRACT TITLE: CHARACTERISATION OF INHIBITOR RESISTANT TEMONIERA (IRT) PRODUCING STRAINS OF ESCHERICHIA COLI AND KLEBSIELLA PNEUMONIAE IN PRETORIA, SOUTH AFRICA}

\section{Introduction}

Beta-lactam/Beta-lactamase inhibitors are extensively used in the treatment of various infections both in the hospital and outpatient settings. Inhibitor resistance may lead to clinical failures in these patients. The aim of this study was to characterise Inhibitor Resistant Temoniera (IRT) producing strains of Escherichia coli and Klebsiella pneumoniae in Pretoria, South Africa.

\section{Methods}

Sixty non-repeat isolates of Escherichia coli and Klebsiella pneumoniae demonstrating inhibitor resistance on the Vitek ${ }^{\circledR} 2$ automated system were collected from 1 December 2013 to 31 July 2015. Manual susceptibility testing for amoxicillinclavulanate was performed using the Kirby Bauer disk diffusion method (CLSI M100S25) and an E-test (bioMeriéux), whilst only the Kirby Bauer disk diffusion method (CLSI M100S25) was used for piperacillin-tazobactam. Genomic DNA was extracted using an automated extraction method. A conventional multiplex PCR assay was performed to detect the Temoniera (TEM), Sulfhydryl Variable (SHV), and Oxacillinase (OXA) genes as described previously. The identity of the amplicons was confirmed using Sanger sequencing.

\section{Results}

Twenty-five (42\%) of the isolates showed a categorical agreement between the E-test and Vitek ${ }^{\circledR} 2$ susceptibility results for amoxicillinclavulanate. The categorical agreement between Vitek ${ }^{\circledR} 2$ and disk diffusion testing for piperacillin-tazobactam was found to be $47 \%$ (28/60). None of the beta-lactamase genes were detected in thirteen (22\%) of the isolates. The TEM gene was detected in $47(78 \%)$ of the isolates. Sequencing confirmed the TEM genes as TEM-1.

\section{Conclusion}

Most of the strains in this study revealed the presence of the TEM-1 gene. No Inhibitor Resistant Temoniera genes were detected in the isolates. Poor categorical agreement $(<50 \%)$ was identified between the Vitek ${ }^{\otimes} 2$ automated system antimicrobial susceptibility testing and manual susceptibility testing. This research gives us some insight into the local epidemiology and resistance mechanisms involved in inhibitor resistance in the Pretoria region.

\section{ID: 8487}

Category: Antimicrobial Resistance (SAASP)

Permission: Yes

Co-Author 1: Sibiya, $T$

Co-Author 1 Institute: Microbiology Laboratory, Charlotte Maxeke Johannesburg Academic Hospital (CMJAH), National Health Laboratory Services

\section{Co-Author 2: Bosman, $\mathrm{N}$}

Co-Author 2 Institute: Microbiology Laboratory, Charlotte Maxeke Johannesburg Academic Hospital (CMJAH), National Health Laboratory Services; Department of Clinical Microbiology and Infectious Diseases (CMID), School of Pathology of the University of the Witwatersrand

Co-Author 3: Perovic, 0

Co-Author 3 Institute: Department of Clinical Microbiology and Infectious Diseases (CMID), School of Pathology of the University of the Witwatersrand; Centre for Healthcare-Associated Infections, Antimicrobial Resistance and Mycoses, National Institute for Communicable Diseases

E-mail Address: thulile.sibiya@nhls.ac.za

Country: South Africa

\section{ABSTRACT TITLE: COMPARISON OF MANUAL VS. RAPID AUTOMATED LABORATORY METHODS FOR THE IDENTIFICATION OF ANAEROBIC BACTERIA ISOLATED FROM CLINICAL SAMPLES}

\section{Introduction}

Laboratory methods used for the identification of anaerobic bacteria isolated from clinical samples have evolved from labour-intense manual methods to more advanced rapid automated systems that have the ability to generate results within minutes as opposed to overnight incubation. The methods used in our laboratory have changed from the manual Finegold and $\mathrm{API}^{\circledR}$ rapid ID $32 \mathrm{~A}$ testing methods used until October 2016, to the automated Vitek ${ }^{\circledast} 2$ ANC card, for two months only, and then Vitek ${ }^{\circledR}$ MS (bioMerieux, France) from November 2016 until the present.

\section{Method}

All anaerobic organisms isolated from clinical samples submitted to our laboratory for identification, from June 2015 to July 2017 were stored in Robertson's cooked meat medium, for a study looking at antimicrobial susceptibility patterns. Anaerobic identification was confirmed using two MALDI-TOF systems, namely the Vitek ${ }^{\circledR}$ MS (bioMerieux, France) and Bruker MALDI Biotyper ${ }^{\circledR}$ (Bruker Daltonics). 


\section{Results}

Of 304 anaerobic organisms stored during the study period, 164 isolates were found to be viable. So far, 139 isolates that were tested using manual methods have been retested using the rapid automated systems for confirmation of identification. Preliminary results show a $94 \%$ concordance. Mixed cultures, with more than one anaerobic organism isolated, were found to be the main cause of discordant results. As a result, Gram staining and further testing will be carried out on these isolates. Final results will be reported once the study is completed.

\section{Conclusion}

The preliminary data show that the rapid automated identification systems introduced recently into our laboratory provide satisfactory results, translating into quicker results for patient management.

\section{ID: 8496}

Category: Antimicrobial Resistance (SAASP)

Permission: Yes

Co-Author 1: de Villiers, B

Co-Author 1 Institute: Sefako Makgatho Health Sciences University

Co-Author 2: le Roux, M

Co-Author 2 Institute: Sefako Makgatho Health Sciences University

Co-Author 3: Ditsele, M

Co-Author 3 Institute: Sefako Makgatho Health Sciences University Co-Author 4: Towobola, 0

Co-Author 4 Institute: Sefako Makgatho Health Sciences University

Co-Author 5: Monokoane, $S$

Co-Author 5 Institute: Sefako Makgatho Health Sciences University

E-mail Address: barbara.devilliers@smu.ac.za

Country: South Africa

ABSTRACT TITLE: AN INVESTIGATION OF TETRACYCLINE-RESISTANT UREAPLASMAS COLONISING PATIENTS AT THE DR GEORGE MUKHARI ACADEMIC HOSPITAL IN 2012 AND 2014

\section{Introduction}

Infections of the female urogenital tract and the foetus/newborn are associated with ureaplasmal colonisation. The tetracycline derivative, doxycycline is prophylactically administered to pregnant women at the Dr George Mukhari Academic Hospital. Resistance to tetracycline was reported among these isolates (SIR Mycoplasma kit) however, no data for MIC values to tetracycline are available.

\section{Methods}

Twenty-six tetracycline-resistant ureaplasmas (isolated from women presenting for termination of pregnancy in 2012 and 2014) were revived from microbank beads in $\mathrm{U} 9$ broth. Positive cultures were inoculated onto A2 agar medium for PCR analysis (speciation;
tetM resistance gene). The MIC to tetracycline was done by broth microdilution.

\section{Results}

Of the 26 Ureaplasma-resistant isolates, only eight could have been revived for further testing. PCR amplification have shown dual infection with Mycoplasma hominis and the presence of the tetM gene in all eight specimens. Broth microdilution results were obtained for four of the 2014 isolates only. Isolates $L 9$ and $L 72$ have shown MIC values of $16 \mu \mathrm{g} / \mathrm{ml}$ and $4 \mu \mathrm{g} / \mathrm{ml}$ respectively. Isolates $\mathrm{L} 18$ and L34 were fully susceptible to tetracycline.

\section{Conclusion}

Dual infection with Mycoplasma hominis was documented for all isolates tested. The promiscuous nature of Tn916 carrying the tetM gene may cause Ureaplasma and Mycoplasma hominis in combination to exhibit an increased resistance to tetracycline. Lack of expression of the tetM gene in isolates L18 and L34 may be clarified by searching for mutations on the structural gene itself or on the leader orf12 peptide. Similar studies on a larger scale should be done to review management strategies for preventing complications in pregnant women across the globe.

\section{ID: 8524}

Category: Antimicrobial Resistance (SAASP)

Permission: Yes

Co-Author 1: Maningi, N

Co-Author 1 Institute: University of Pretoria

Co-Author 2: Fourie, B

Co-Author 2 Institute: University of Pretoria

E-mail Address: nontuthuko.maningi@up.ac.za

Country: South Africa

\section{ABSTRACT TITLE: MUTATION ARG463LEU OF THE KATG GENE IN NON-VIABLE MULTIDRUG RESISTANT TUBERCULOSIS ISOLATES FROM SOUTH AFRICA}

\section{Introduction}

Tuberculosis is a major public health problem. In 2015 the TB death rate was 1.5 million cases compared to the HIV death rates of 1.2 million worldwide. Mutation Arg463Leu is not targeted in our current laboratory diagnostic commercial assay for MDR-TB. Only codon 315 mutation and inhA mutations are on the assay. Mutations in codon 463 have been shown to be responsible for 20 to $40 \%$ of the INH phenotypic low-level resistance. The frequency patterns of mutations that lead to Isoniazid (INH) resistance vary within the individual genes and geographically areas.

\section{Methods}

Next generation sequencing, the Illumina Miseq was performed in this study on 100 phenotypic MDR-TB samples. Spoligotyping was performed to assess the association of mutation Arg463Leu with MTB lineages. 


\section{Results}

In this study mutation, Arg463Leu was responsible for INH resistance in 14\% (14/100) of our MDR-TB cultures in the absence of codon 315 mutation. This could mean that we are currently missing about $14 \%$ of INH resistance when diagnosing MDR-TB patients. The lineage type of thirteen of the fourteen isolates with R463L mutations was Beijing and one was X3.

\section{Conclusion}

Discriminatory power will be achieved if this target is considered for inclusion in new rapid molecular tests for improved molecular detection of MDR-TB. Mutation Arg463Leu was more associated with the virulent Beijing strains in this study. This was the first study in South Africa to investigate the association of katG codon 463 mutations with INH phenotypic resistance in MDRTB strains from Western Cape and Gauteng Provinces

\section{ID: 8547}

Category: Antimicrobial Resistance (SAASP)

Permission: Yes

Co-Author 1: Saaiman, A

Co-Author 1 Institute: Netcare

Co-Author 2: Corder, P

Co-Author 2 Institute: Netcare

Co-Author 3: Trauernicht, $T$

Co-Author 3 Institute: Netcare

Co-Author 4: van der Watt, $R$

Co-Author 4 Institute: Netcare

E-mail Address: annerie.saaiman@netcare.co.za

Country: South Africa

\section{ABSTRACT TITLE: ENSURING IMPROVEMENT IN ANTIMICROBIAL HANG TIME THROUGH A MULTI- DISCIPLINARY APPROACH}

\section{Introduction}

Hang time is measuring the time elapsed from a physician prescribing an antimicrobial agent, until administration. According to Kumar et al., 2006, each hour of delay in the administration of antimicrobials following a doctor's prescription is associated with an average decrease in patient survival of $7.6 \%$ per hour in patients with sepsis. Administration of antimicrobials within the first hour is associated with $80 \%$ increased possibility of survival. The aim of the study was to determine improvement in hang time compliance, over a period of 52 weeks involving pharmacy, nursing, doctors and hospital management.

\section{Method}

Hang time on first dose antimicrobials was measured across all wards, during daily ward rounds from 4 July 2016 to 2 July 2017. The ward pharmacists collected data on patients who were on specific target antibiotics, using the antimicrobial prescription charts. Data was captured on an electronic surveillance system.

Weekly hang time compliance reports were compiled for the hospital and per unit. The reports were distributed on a monthly basis to the nursing manager and pharmacy manager. The nursing manager distributed the hang time compliance graphs to each unit manager.

Commonly used antimicrobials were made available as ward stock in each unit. Hang time posters were placed in each unit, to create awareness and a SOP on antimicrobial hang time was formulated and approved.

\section{Result}

A total of 2039 cases were assessed for compliance over a period of 52 weeks and $76.56 \%$ of the cases received their initial dose of antimicrobials within an hour following a prescription. The median hang time compliance increased from $72.15 \%$ in week eight to $89.57 \%$ in week 52 .

\section{Conclusion}

This antimicrobial stewardship project needs constant monitoring, commitment and a multi-disciplinary approach to ensure sustainability. Improvement work is required to increase compliance to ensure all patients receive their first antimicrobial dose within an hour following a prescription.

\section{ID: 8553}

Category: Antimicrobial Resistance (SAASP)

Permission: Yes

Co-Author 1: Paterson, L

Co-Author 1 Institute: Stellenbosch University

Co-Author 2: Whitelaw, PA

Co-Author 2 Institute: Stellenbosch University

Co-Author 3: Newton-Foot, DM

Co-Author 3 Institute: Stellenbosch University

E-mail Address: 16491270@sun.ac.za

Country: South Africa

\section{ABSTRACT TITLE: THE EPIDEMIOLOGY OF GRAM- NEGATIVE BACTERAEMIA AT TYGERBERG HOSPITAL}

\section{Introduction}

E. coli and K. pneumoniae are common causes of bacteraemia. $\beta$-lactam antibiotics are used to treat these infections. Common cephalosporin and carbapenem-resistance mechanisms include extended spectrum $\beta$-lactamases (ESBLs) and carbapenemases respectively. ESBLs belong to three major families: TEM, SHV and CTX-M. There is limited local data describing ESBL distribution. This is important for surveillance and evaluating infection prevention practices. 


\section{Methods}

Bacteraemic E. coli $(n=70)$ and K. pneumoniae $(n=70)$ isolates were collected from the NHLS Microbiology laboratory between April 2015 and March 2016. Identification and antibiotic susceptibility testing (AST) were performed as part of routine testing. Carbapenemase and ESBL genes were detected by PCR, and CTX-M genes characterised by PCR. Isolates were typed using rep-PCR, and images analysed using Gel compar.

\section{Results}

Thirty-one percent (44/140) of patients were paediatric. Patients were located in wards throughout the hospital. Fifty-four percent (38/70) of $K$. pneumoniae and $16 \%(11 / 70)$ of E. coli were cephalosporin resistant (presumed ESBL). None were carbapenem resistant. There was good correlation between phenotypic AST and ESBL PCR. No isolates contained carbapenemase genes. TEM and SHV were the commonest $\beta$-lactamases in E. coli (49/70; 70\%) and K. pneumoniae $(59 / 70 ; 84,3 \%)$ respectively. Multiple genes were present in 42/70 (60\%) of $K$. pneumoniae isolates, and only 4/70 $(5,7 \%)$ of $E$. coli isolates. Of the 46 CTX-M genes, 43 were group 1 , two were group 9, and one was untypeable. Strain typing showed substantial diversity among $E$. coli and K. pneumoniae, with minimal clustering. There was no association between clusters and hospital wards or ESBL type.

\section{Conclusion}

While the commonest $\beta$-lactamases were TEM- and SHV-related, additional work is needed to further classify these genes. The lack of clustering suggests multiple clones in the hospital. Differentiation of hospital and community acquired infection, and additional molecular typing is planned to further investigate this.

\section{ID: 8533}

Category: Antimicrobial Resistance (SAASP)

Permission: Yes

Co-Author 1: Khan, Y

Co-Author 1 Institute: Nelson Mandela University

Co-Author 2: Coetzee, $\mathrm{R}$

Co-Author 2 Institute: University of Western Cape

Co-Author 3: McCartney, J

Co-Author 3 Institute: Nelson Mandela University

Co-Author 4: Boschmans, $S$

Co-Author 4 Institute: Nelson Mandela University

E-mail Address: s213227177@nmmu.ac.za

Country: South Africa

\section{ABSTRACT TITLE: AN APPROACH TO DETERMINING A BASELINE SOUTH AFRICAN ANTIMICROBIAL STEWARDSHIP CURRICULUM FOR THE BACHELOR OF PHARMACY PROGRAMME}

\section{Introduction}

Antimicrobial stewardship (AMS) is a rapidly developing healthcare practice which aims to ensure judicious use of antimicrobial agents, to minimise the development of antimicrobial resistance. During a workshop at the 37th annual general meeting of the Academy of Pharmaceutical Sciences of South Africa, AMS training for South African pharmacy students was discussed. This study evolved from that workshop and involves the development of a proposed AMS curriculum, suitable for implementation in undergraduate pharmacy programmes, in South Africa.

\section{Method}

The study aims to ensure that the proposed curriculum is inline with international and national AMS recommendations, and incorporates input from AMS professionals in South Africa. The methodology involves several phases, including: a desktop review; consultation with academic pharmacists from South African universities; and consultation with AMS experts in South Africa (infectious disease specialists, clinical microbiologists and AMS pharmacists). Collation of the results from these study phases will allow for the development of a final proposed AMS curriculum.

\section{Result}

The phase one desktop evaluation results have revealed the content and structure of international AMS curricula, as well as the principles which govern AMS in South Africa. Frequency counts have been performed to determine the incidence of trends in the literature. The results indicate that international AMS curricula consist of ten to thirty-hour programmes, which utilise mixed pedagogies and multiple evaluation techniques. The content of these international curricula are in-line with international and national AMS principles. Identification of South African AMS strategic objectives, enablers and principles has further contextualised the initial proposed content for the curriculum being developed.

\section{Conclusion}

The findings from the desktop evaluation will form the groundwork of the AMS curriculum to be developed. Collation of these findings with future study phases will allow for the formation of the final proposed AMS curriculum, suitable for incorporation into South African undergraduate pharmacy programmes.

\section{ID: 8598}

Category: Antimicrobial Resistance (SAASP)

Permission: Yes

Co-Author 1: Skinner, DL

Co-Author 1 Institute: University of KwaZulu-Natal, Department of Anaesthesiology and Critical Care

Co-Author 2: Ramjathan, P

Co-Author 2 Institute: University of KwaZulu-Natal, School of Laboratory Medicine \& Medical Sciences

Co-Author 3: Mlisana, K 
Co-Author 3 Institute: University of KwaZulu-Natal, School of Laboratory Medicine \& Medical Sciences

E-mail Address: drdavidskinner@gmail.com

Country: South Africa

\section{ABSTRACT TITLE: SPECTRUM AND SUSCEPTIBILITY PROFILE OF MICROORGANISMS CAUSING PERITONITIS IN CRITICALLYILL PATIENTS}

\section{Introduction}

The incidence of drug resistant infections in critically ill patients is reported to be increasing worldwide, including South Africa. Data has shown that patients undergoing re-laparotomy have increased rates of drug resistant bacteria.

The aim of this study was to describe the susceptibility profile of microorganisms found during laparotomy for peritonitis in a group of critically ill patients.

\section{Methods}

The study included 35 isolates obtained from critically ill patients in King Edward VIII Hospital ICU. Peritoneal fluid data was extracted retrospectively from the laboratory electronic database for the period 1 January to 31 December 2016. To describe the spectrum of organisms only one of the same isolate per patient was counted.

\section{Results}

A total of 35 isolates were obtained from 24 patients $(n=34$ from relaparotomy). The most common abdominal pathologies ranged from secondary peritonitis following trauma $(n=7)$, peptic ulcer disease $(n=5)$ and iatrogenic bowel injury $(n=5)$.

The predominant microorganisms isolated were Gram-negative bacteria ( $\mathrm{n}=19,54.3 \%$ ) followed by Gram-positive cocci $(n=12,34.3 \%)$ and Candida species $(n=4,11.4 \%)$. The commonest Gram-negative bacteria were Klebsiella pneumonia e $(\mathrm{n}=5,143 \%)$, Acinetobacter baumanii $(n=5,14,3 \%)$ and the commonest Grampositive was Enterococcus species $(n=6,17.1 \%$ ) There were high rates of resistance in the enterobacteriaceae isolated, with only $8 \%$ being susceptible to amoxicillin-clavulanate; $75 \%$ susceptible to imipenem and amikacin; $42 \%$ susceptible to piperacillintazobactam and gentamycin. 50\% of Enteroccocci were susceptible to ampicillin. $75 \%$ of yeasts isolated were non-albicans Candida species. Acinetobacter species isolated were multidrug resistant with only $40 \%$ and $20 \%$ susceptibility to gentamycin and amikacin respectively.

\section{Conclusion}

The high rates of drug resistant bacteria would impact on morbidity of patients. Broad spectrum cover for Gram-positive, Gram-negative bacteria and possibly antifungal therapy would be required for these patients as empiric therapy. The high incidence of nonalbicans Candida species is a cause for concern. Final treatment would need to be guided by culture results.

\section{ID: 8297}

Category: Sexually Transmitted Diseases (STDSSA)

Permission: Yes

Co-Author 1: Mzobe, G

Co-Author 1 Institute: University of KwaZulu-Natal

Co-Author 2: Sturm, W

Co-Author 2 Institute: University of KwaZulu-Natal

Co-Author 3: Joubert, B

Co-Author 3 Institute: University of KwaZulu-Natal

E-mail Address: gugulethu.mzobe@caprisa.org

Country: South Africa

\section{ABSTRACT TITLE: TEMPORAL GENE EXPRESSION OF CHLAMYDIA TRACHOMATIS IN KERATINOCYTES AT $370 C$ VERSUS $330 C$}

\section{Introduction}

Keratinocytes are the first port of entry for Chlamydia trachomatis of the lymphogranuloma venereum (LGV) biovar which causes LGV. However, LGV pathogenesis and gene expression studies are usually performed in cells which are not the native host cells.

\section{Methods}

To investigate the effect of temperature on the expression of chlamydial genes, $\mathrm{HaCaT}$ cells were grown in 12 well plates and inoculated with chlamydia (L2, E and US151) and incubated at $330 \mathrm{C}$ or $370 \mathrm{C}$. At 2, 12, 24, 36 and 48 hpi (also 60 and 72 hpi for cells incubated at $33^{\circ} \mathrm{C}$ ), total RNA was harvested and converted to CDNA which was then analysed by RT PCR for the expression of groEL-1, incB, pyk, hctA and omcB. TEM was performed to confirm the stage of the developmental cycle that the organism was at inside the keratinocytes.

\section{Results}

RT-PCR analysis showed that the early cycle genes were expressed throughout the cycle at $370 \mathrm{C}$ and $330 \mathrm{C}$ as expected. At $37 \mathrm{oC}$, the mid- and late-cycle genes were expressed throughout the cycle, with different patterns between the isolates tested. At 33oC, the mid cycle genes also showed different patterns of expression between the tested isolates. However, the expression of late cycle genes in L2 and $\mathrm{E}$ was in keeping to that in the published literature.

\section{Conclusion}

This study confirms that mid- and late-cycle chlamydial gene expression levels are different to the published research and that temperature has an effect on the level of chlamydial gene expression when grown in keratinocytes.

\section{ID: 8417}

Category: Sexually Transmitted Diseases (STDSSA)

Permission: Yes

Co-Author 1: Majola, MS 
Co-Author 1 Institute: University of Pretoria

Co-Author 2: Lombaard, HA

Co-Author 2 Institute: University of the Witswatersrand

Co-Author 3: Peters, RPH

Co-Author 3 Institute: University of Pretoria

Co-Author 4: Ehlers, MM

Co-Author 4 Institute: National Health Laboratory Service; University of Pretoria

Co-Author 5: Redelinghuys, MJ

Co-Author 5 Institute: University of Pretoria

Co-Author 6: Kamutando, CN

Co-Author 6 Institute: University of Pretoria

Co-Author 7: Valverde, $A$

Co-Author 7 Institute: University of the Free State

Co-Author 8: Kock, MM

Co-Author 8 Institute: National Health Laboratory Service; University of Pretoria

E-mail Address: sweetnessmapule651@gmail.com

Country: South Africa

\section{ABSTRACT TITLE: VAGINAL MICROBIOME PROFILING OF WOMEN WITH AND WITHOUT BACTERIAL VAGINOSIS USING ILLUMINA TARGTED SEQUENCES}

\section{Introduction}

Bacterial vaginosis (BV) is the most prevalent dysbiosis that is characterised by a shift from a normal vaginal microbiome (mainly lactobacilli producing lactic acid and hydrogen peroxide) to a polymicrobial mixture of pathogens. Bacterial vaginosis (higher prevalence in black women) has negative impacts on maternal (e.g. increased risk for human immunodeficiency virus acquisition) and foetal health (e.g. premature rupture of the membranes). The vaginal microbiome can be profiled using next generation sequencing platforms like Illumina MiSeq. The aim of this study was to determine the alpha diversity (richness and evenness) of the vaginal microbiome of African women with and without BV and to identify the risk profiles associated with BV and HIV.

\section{Methods}

Bacterial vaginosis status was diagnosed using Nugent scoring (categories: healthy, intermediate and BV) of Gram-stained slides. Forty-one samples were selected for targeted 165 metagenomics based on the HIV and BV status. Illumina MiSeq sequencing was done by the Agricultural Biotechnology Sequencing Platform. The metagenomic data was analysed using Quantitative Insight into Microbial Ecology (QIIME) and statistical analysis was done using R.

\section{Results}

A total of 2489715 high quality reads was generated from all samples with an average of 60724.756 Operational taxonomic units (OTUs) per sample. Lactobacillus iners was the most dominant species in BV negative women while the Prevotella species (spp.) was the most dominant in BV positive women.

\section{Conclusion}

Lactobacillus spp. are known to produce hydrogen peroxide, which prevents vaginal colonisation by pathogens. Prevotella spp. such as Prevotella bivia is an opportunistic organism known to invade epithelial cells causing inflammatory responses and endometritis. Therefore, its presence in the vagina warrants efforts to treat and eradicate it.

\section{ID: 8549}

Category: Sexually Transmitted Diseases (STDSSA)

Permission: Yes

Co-Author 1: Nemarude, L

Co-Author 1 Institute: Sefako Makgatho Health Sciences University, Department of Microbiological Pathology

Co-Author 2: Mashishi, $\mathrm{T}$

Co-Author 2 Institute: Sefako Makgatho Health Sciences University, Department of Virological Pathology

Co-Author 3: Tiiti, $T$

Co-Author 3 Institute: Sefako Makgatho Health Sciences University, Department of Virological Pathology

Co-Author 4: Selabe, G

Co-Author 4 Institute: Sefako Makgatho Health Sciences University, Department of Virological Pathology

Co-Author 5: Bogers, JP

Co-Author 5 Institute: University of Antwerp, AMBIOR, Belgium

Co-Author 6: Nchabeleng, $M$

Co-Author 6 Institute: Sefako Makgatho Health Sciences University, Department of Microbiological Pathology

Co-Author 7: Lebelo, $R$

Co-Author 7 Institute: Sefako Makgatho Health Sciences University, Department of Virological Pathology

E-mail Address: ramslisbeth@yahoo.com

Country: South Africa \& Belgium

ABSTRACT TITLE: HPV INFECTIONS AND CO-INFECTION WITH SELECTED SEXUALLY TRANSMITTED INFECTIONS IN WOMEN ATTENDING DGMAH, PRETORIA, SOUTH AFRICA

\section{Introduction}

Cervical cancer is the fourth most common cancer and the fourth most prevalent cause of death in women worldwide. Persistent high-risk HPV infection has been established as a strong risk factor for progression of cervical precursor lesions to cervical cancer. Cofactors such as sexually transmitted infections and bacterial vaginosis have been considered as important cofactors for development of cervical cancer. These STIs may affect clearance of $\mathrm{HPV}$ infection resulting in persistent HPV infection.

\section{Methods}

This is a cross-section ongoing study among women aged 18 years and older. Self-collected (vaginal) and doctor (cervical) liquid-based 
cytology (LBC) samples are collected thus far; results for 64 patients are analysed. HPV detection was performed using Abbott m2000rt system and detection of other STIs was performed with Anyplex II STI-7 that identifies seven major STIs: Chlamydia trachomatis (CT), Trichomonas vaginalis (TV), Mycoplasma homonis (MH), Mycoplasma genitalium (MG), Ureaplasma urealyticum (UU), Ureaplasma parvum (UP), Neisseria gonorrhoeae (NG).

\section{Results}

Only samples positive for HPV DNA were tested for the other STIs. In self-collected samples, $51.8 \%$ were positive for UP, $57.4 \%$ were $\mathrm{MH}$, $11.1 \%$ were TV, $24.1 \%$ were UU, $5.6 \%$ were $\mathrm{CT}, 1.9 \%$ were $\mathrm{MG}, 10$ of the self-collected samples were not matched with doctor-collected samples. In the doctor-collected LBC samples, $47.3 \%$ were positive for UP, $45.5 \%$ for $\mathrm{MH}, 10.9 \%$ for TV, $18.2 \%$ for UU, $7.3 \%$ for CT and $5.5 \%$ for MG. STIs multiple infections were detected in $68.5 \%$ of the samples.

\section{Conclusion}

Almost half of the patients infected with HPV were infected with MH and UP. These two STIs were the common STIs in both the vaginal and cervical LBC samples. The least detected was MG in both types of samples. Co-infection with these STIs may provide an environment $t$ for HPV infection to persist.

\section{ID: 8376}

Category: Travel Medicine (SASTM)

Permission: Yes

Author: Winkler, M

Author Institute: none

E-mail Address: MAXWINK@GMAIL.COM

Country: South Africa

\section{ABSTRACT TITLE: A BIBLIOMETRIC ANALYSIS OF YELLOW FEVER, DENGUE, CHIKUNGUNYA AND ZIKA RESEARCH TRENDS FROM 2000 TO 2016}

\section{Introduction}

A bibliometric analysis was conducted to investigate trends in Yellow Fever, Dengue, Chikungunya and Zika research output from 2000 to 2016. For each illness, this included a) description of the number of original research publications published per year; b) analysis of the geographical origins of the research during the defined study period; and c) identification of the top ten most cited articles per illness topic.

\section{Methods}

Using the Web of Knowledge Database (https://webofknowledge. com), we searched for any original research articles published between 2000 to present which contained "Yellow Fever", "Dengue", "Chikungunya" or "Zika" search terms in the title. The built-in analysis features were used to sort these results by year of publication, geographical origin and number of citations.

\section{Results}

According to the defined search term criteria, Dengue had the most publications during the study period ( 6403 articles), followed by Chikungunya (1 040 articles), Yellow Fever (746 articles) and Zika (417 articles). While the number of Dengue research articles has increased steadily during the study period (14\% per year), Yellow Fever research articles have remained relatively static since 2000 . Chikungunya research articles show two peak publication periods: 2005-2008 and 2013-present. The first Zika research articles were published in 2008 and $91 \%$ of all Zika research articles were published in 2016. For each illness, the USA contributed the greatest number of research articles (24-51\%). While developing countries with an endemic illness burden such as Brazil and India also feature prominently, there is a distinct lack of research output from Africa.

\section{Conclusion}

While overall research output for each illness appears to be related to disease prevalence, other factors such as recent outbreaks, increased public awareness and global access to research funding may influence research trends.

\section{ID: 8322}

Category: Antimicrobial Resistance (SAASP)

Permission: Yes

Co-Author 1: Naidoo, N

Co-Author 1 Institute: University of KwaZulu-Natal

Co-Author 2: Pillay, B

Co-Author 2 Institute: University of KwaZulu-Natal

Co-Author 3: Bubb, M

Co-Author 3 Institute: National Bioproducts Institute

Co-Author 4: Kumar, A

Co-Author 4 Institute: University of KwaZulu-Natal

Co-Author 5: Chiliza, T

Co-Author 5 Institute: University of KwaZulu-Natal

Co-Author 6: Pillay, M

Co-Author 6 Institute: University of KwaZulu-Natal

E-mail Address: pillay2@gmail.com

Country: South Africa

\section{ABSTRACT TITLE: SIGNAL PEPTIDE PREDICTION SUGGESTS MYCOBACTERIUM TUBERCULOSIS CURLI PILIN SUBUNIT SECRETION VIA THE SEC PATHWAY MAY HINDER MTP OVEREXPRESSION IN ESCHERICHIA COLI}

Mycobacterium tuberculosis curli pili (MTP) are novel, potential TB diagnostic biomarkers. However, the production of high quality recombinant transmembrane and secretory proteins may be challenging due to their secretion attributes. The signal peptide of MTP governed by the classical secretion pathway may hinder the purification of the protein in E. coli. In this study, the secretion 
characteristics of MTP were determined and cloning, expression and purification of MTP was attempted in E.coli. A fragment of MTP unique to MTBC was cloned into pet101 and pGEX-6P-1 vectors and confirmed by nucleotide sequencing. Expression of the protein was attempted at IPTG concentrations ranging from $0.1 \mathrm{mM}$ to $1 \mathrm{mM}$ and at temperatures between $25^{\circ} \mathrm{C}$ to $37^{\circ} \mathrm{C}$. The pGEX-6P-1/mtp clone expressed protein was purified, yielding a MTP-GST fusion protein and a free GST band that were analysed by LC/MS mass spectrometry. Inclusion body preparation attempted from the pet101/mtp clone yielded two bands at $10 \mathrm{kDa}$ and below $10 \mathrm{kDa}$, both of which were analysed by LC/MS mass spectrometry. Transcription activity clones was interrogated by real time PCR. Signal peptide and protein secretion characteristics were determined by bioinformatics analysis. Expression of the pGEX-6P-1/mtp clone demonstrated the expected fragment at approximately $35 \mathrm{kDa}$, confirmed by Western Blotting but mass spectrometry did not detect any MTP fragments. The bioinformatics analyses of MTP predicted a strong Sec regulated secretion pathway and the absence of non-classical "mycobacterial specific" secretion. Excluding the signal peptide region and using a GST tag greatly enhanced the expression of the protein in the soluble fraction. However, purification of the MTP peptide remained problematic. The predicted Sec regulated secretion pathway may play a role in the inhibition of MTP overexpression in E.coli. Alternatives to E. coli expression or more efficient purification strategies are required for the acquisition of high quality $M$. tuberculosis antigens.

\section{ID: 8387}

Category: Antimicrobial Resistance (SAASP)

Permission: Yes

Co-Author 1: Shallouf, M

Co-Author 1 Institute: University of the Western Cape

Co-Author 2: Abrantes, PMDS

Co-Author 2 Institute: University of the Western Cape

Co-Author 3: Fielding, B

Co-Author 3 Institute: University of the Western Cape

Co-Author 4: Africa, CWJ

Co-Author 4 Institute: University of the Western Cape

E-mail Address: mohamed.shallouf@gmail.com

Country: South Africa \& Libya

\section{ABSTRACT TITLE: COLISTIN, CARBAPENEM AND CEPHALOSPORIN-RESISTANT KLEBSIELLA PNEUMONIAE REPORTED FROM MISRATA, LIBYA}

\section{Introduction}

Klebsiella pneumoniae is a significant human pathogen causing community and nosocomially-acquired infections. The spread of extended-spectrum $\beta$-lactamase (ESBL)-producing enterobacteriaceae has resulted in the emergence of carbapenemresistant $K$. pneumoniae (CRKP). Although colistin is generally used to treatment CRKP infections, resistance to colistin has also been reported in several countries and become a major public health concern.

\section{Method}

Clinical samples were obtained from hospitalised (140) and nonhospitalised patients (60) in Misrata, Libya. Identification of the isolated species was achieved using the VITEK 2 compact system. Screening for carbapenem and cephalosporin-resistance was performed using the disk diffusion method with carbapenem $10 \mu \mathrm{g}$ and cephalosporin $30 \mu \mathrm{g}$ disks and minimum inhibitory concentrations (MIC) determined by VITEK 2. Colistin resistance was determined using both Sensititre Gram-negative Xtra plate format (GNX2F) and VITEK 2. Carbapenemase activity was detected using the RAPIDEC CARBA NP, Modified Hodge test, carbapenem inactivation methods, MAST Combi Carba plus kit (D73C) and meropenem combined disc test. ESBL production was confirmed using Sensititre ESBL confirmatory plates (ESB1F), modified double disc synergy test (MDDST) and MAST ESBL detection kit D52C.

\section{Result}

Of the eighty K. pneumoniae isolates, $47(58,75 \%)$ demonstrated resistance to at least one of the four carbapenems, 16 (20\%) were ESBL producers, and 2 (2.5\%) were carbapenem and colistin resistant. Seventeen (21.25\%) isolates were susceptible to all antibiotics tested except ampicillin and augmentin.

\section{Conclusion}

This study has revealed the high rate of CRKP among hospitalised patients in Libya with OXA-48 being the most prevalent. The emerging colistin resistance and the spread of ESBL producers in both the hospital and in the community requires urgent action such as the establishment of antibiotic stewardship programmes along with firm infection control practices to prevent further spread in both the clinical and non-clinical settings.

This study was funded by the Libyan government.

\section{ID: 8455}

Category: Antimicrobial Resistance (SAASP)

Permission: Yes

Co-Author 1: Said, H

Co-Author 1 Institute: Centre for Tuberculosis, National Institute of Communicable Diseases

Co-Author 2: Omar, $\mathrm{S}$

Co-Author 2 Institute: Centre for Tuberculosis, National Institute of Communicable Diseases

Co-Author 3: Fallows, D

Co-Author 3 Institute: Public Health Research Institute, Rutgers University, Newark, NJ

Co-Author 4: Rukasha, I

Co-Author 4 Institute: Centre for Tuberculosis, National Institute of Communicable Diseases

Co-Author 5: Ismail, N

Co-Author $\mathbf{5}$ Institute: Centre for Tuberculosis, National Institute 
of Communicable Diseases; Department of Medical Microbiology, Faculty of Health Science, University of Pretoria

E-mail Address: halimas@nicd.ac.za

Country: South Africa \& The United States of America

\section{ABSTRACT TITLE: RESISTANCE TO SECOND-LINE ANTI TB DRUGS IN SOUTH AFRICA: ASSOCIATION BETWEEN MIC AND GENETIC RESISTANCE DETERMINANTS}

\section{Background}

Genetic polymorphisms among Mycobacterium tuberculosis are known to confer differential levels of resistance. We investigated the association between minimum inhibitory concentration (MIC) levels and mutations in gyrA and gyrB (OFX and MOX) and rrs (AMI and KAN) genes.

\section{Method}

The presence of mutations in 210 drug-resistant $M$. tuberculosis isolates was determined by DNA sequencing and the MICs were quantified by Sensititer MYCOTB broth microdilution method.

\section{Result}

In total, 35 isolates were resistant to OFX, 30 to MOX at $>0.5 \mu \mathrm{g} / \mathrm{ml}$ breakpoint and only two at $>2 \mu \mathrm{g} / \mathrm{ml}$ breakpoint. The gyrA mutations with A90V, D94G, D94N, S91P were detected in 13,11, 1, 2 and 7, 17,1, 1 of the OFX and MOX resistant isolates respectively. Isolates with mutations at the codon 90 and 91 had MIC of $4-8 \mu \mathrm{g} / \mathrm{ml}$ for OFX and $1 \mu \mathrm{g} / \mathrm{ml}$ for MOX, respectively, and isolates with mutations at codon 94 had MIC of $8-32 \mu \mathrm{g} / \mathrm{ml}$ and $1-8 \mu \mathrm{g} / \mathrm{ml}$ for OFX and MOX, respectively. The L566F gyrB mutation were found in two OFX and MOX resistant isolates (MIC $=8 \mu \mathrm{g} / \mathrm{ml}$ ). The two MOX-resistant isolates at $>2 \mu \mathrm{g} / \mathrm{ml}$ lbreakpointhadmutationatthecodon94andwereassociated with high MIC $(\geq 8)$. Thirteen isolates showed resistance to AMI and $\mathrm{KAN}$, with 11 isolates showing cross resistance. The majority of AMK $(n=13)$ and KAN $(n=8)$ resistant isolates harboured a mutation in the rrs locus. All isolates with mutations at A1401G (6/6) and a double mutation (A514C_A1401G) were found to have a high MIC $(16 \mu \mathrm{g} / \mathrm{ml})$ for AMl as well as for KAN $(40 \mu \mathrm{g} / \mathrm{ml})$.

\section{Conclusions}

MTB MICs were found to be consistently lower for MOX than for OFX among isolates with the same gyrA mutation. The codon 94 gyrA and L566F gyrB mutation was associated with high-level resistance to both OFX and MOX. This finding indicates moxifloxacin may be used in the treatment of ofloxacin-resistant TB. Mutation at A1401G of the rrs gene was associated with high-level of resistance conferring cross-resistance between KAN and AMI.

\section{ID: 8451}

Category: Antimicrobial Resistance (SAASP)

Permission: Yes

Co-Author 1: Musyoki, A

Co-Author 1 Institute: Sefako Health Sciences University
Co-Author 2: Maluleka, C

Co-Author 2 Institute: Sefako Makgatho Health Science University; National Health Laboratory Service

E-mail Address: pamusyoki@gmail.com

Country: South Africa

ABSTRACT TITLE: THE FIRST EVER REPORT ON
HAEMOPHILUS INFLUENZAE CARRIAGE IN CHILDREN
ATTENDING DGMAH: BIG BOOST FOR HIB VACCINATION

\section{Introduction}

In 1999, South Africa incorporated Haemophilus influenzae type b (Hib) vaccine in the infant routine immunisation programme which substantially reduced the rate of mortality and morbidity due to Hib in the country. However, prevalence of $H$. Influenzae has not been evaluated at Dr George Mukhari Academic Hospital (DGMAH) postintroduction of the vaccine. Reports from other parts of the world have reported mixed findings on carriage and type replacement. Hence, this study aimed to determine the prevalence of $H$. Influenzae carriage in the nasopharynx of children served by DGMAH.

\section{Methods}

A total number of 350 nasopharyngeal swabs were collected from children aged between two months to 14 years attending the paediatric clinic at DGMAH. The samples were processed in the laboratory using various methods. These included phenotypic traits identification such as colony morphology on chocolate agar, Gram staining, oxidase production and tests on growth factors requirement. Lastly, the identification of isolates was confirmed by conventional polymerase chain reaction targeting the omp6 gene region.

\section{Results}

Of the 350 samples processed, 50 isolates were Haemophilus species positive by phenotypic test methods. Of which, $28(8 \%)$ isolates were confirmed positive for $\mathrm{H}$. Influenzae by PCR, whereas 322 (92\%) were found to be negative for $H$. Influenzae. The first ever electron microscopy pictures of $H$. influenzae isolates at DGMAH showed the characteristic pleomorphic shape of $H$. Influenzae.

\section{Conclusion}

The study demonstrated an $8 \% \mathrm{H}$. Influenzae carriage rate in children between the ages of two months to 14 years in DGMAH.

\section{ID: 8467}

Category: Antimicrobial Resistance (SAASP)

Permission: Yes

Co-Author 1: Sheik, NB

Co-Author 1 Institute: National Renal Care

Co-Author 2: Clark, C

Co-Author 2 Institute: National Renal Care

Co-Author 3: Fabian, J 
Co-Author 3 Institute: Wits Donald Gordon Medical Centre

E-mail Address: naseema.sheik@nrc.co.za

\section{ABSTRACT TITLE: CHRONIC DIALYSIS THERAPY YIELDS A SIGNIFICANT INFECTIOUS BURDEN}

\section{Introduction}

Patients receiving chronic dialysis therapy are vulnerable to infection because of regular contact with hospital environments, indwelling catheters for dialysis access and comorbidity such as diabetes.

\section{Methods}

A retrospective review was conducted of laboratory-confirmed infections in patients on peritoneal dialysis (PD) or haemodialysis (HD) from June 2015 to May 2016. The profile of Gram-positive, Gram-negative bacterial pathogens and fungal pathogens was described and antimicrobial susceptibility patterns were evaluated.

\section{Results}

Two-hundred-and-thirty-eight infections were reported, corresponding to an infection rate of 9.1/100 dialysis patient years (95\% Cl: 8.0-10.4). The infection rate for PD was $31.3 / 100$ dialysis patient years $(95 \% \mathrm{Cl} 24.2-39.8)$ which was significantly higher than that for $\mathrm{HD}(7.2 / 100$ dialysis patient years $(95 \% \mathrm{Cl} 6.2-8.3))$ $(p<0.0001)$. Fifty-one bloodstream infections were reported in the HD group and 41 cases of peritonitis in the PD group. There were marked differences in infection rates when comparing regions and units across South Africa.

In those who developed infections, one third had a hospital admission in the last 90 days; $34 \%$ were diabetic; mean age at time of infection was 54.3 years. $47.9 \%$ (114) were hospitalised for the infection with a median length of stay of seven days and a $5.0 \%$ ( $n=12$ ) mortality. $49.0 \%$ of the patients had Gram-positive infections, $44.4 \%$ had Gram-negative infections, and two patients had fungal infections.

\section{Conclusion}

This is the first published study from a renal dialysis company in SA. The results demonstrate a significant burden of both Gram-positive and -negative organisms and half of these organisms exhibit antimicrobial resistance profiles.

\section{ID: 8511}

Category: Antimicrobial Resistance (SAASP)

Permission: Yes

Author: Nxele, N

Author Institute: University of KwaZulu-Natal

E-mail Address: 209528542@stu.ukzn.ac.za

Country: South Africa

\section{ABSTRACT TITLE: DETECTION OF HUMAN NOROVIRUSES AMONG CHILDREN FIVE YEARS AND UNDER IN KWAZULU-NATAL}

\section{Introduction}

Noroviruses (NoV) are single-stranded RNA viruses belonging to the family caliciviridae. They cause gastroenteritis in all age groups but mostly in young children and the elderly. They are classified into seven genogroups (GI-GVII) and only GI, GIl and GIV infect humans. They cause self-limiting infection that resolves in 10-51 hours after exposure and symptoms include diarrhoea, vomiting, cramps, chills and headaches. The aim of this study was to detect NoV GI and GII in children $\leq 5$ years in KwaZulu-Natal.

\section{Methods}

Stool specimen were collected in children aged five years and younger with diarrhoeal symptoms in King Edward VIII Hospital. The specimen was tested for NoV antigen using ELISA, and RT-qPCR was used to detect viral RNA. The result was compared to each other.

\section{Results}

One-hundred-and-eighty-two specimens were tested for NoV by ELISA and RT-qPCR method. Compared to RT-qPCR, the ELISA had a sensitivity of $24.39 \%(95 \% \mathrm{Cl}=12.36-40.30 \%)$ and specificity of $93.62 \%$ ( $95 \% \mathrm{Cl}=88.23-97.04 \%)$. The incidence of NoV when specimens were tested by RT-qPCR was $22.5 \%$. Infection rate was higher in children between the age of 12-24 months and there was no statistical significant between females and males.

\section{Conclusion}

NoV was a common cause of diarrhoeal illness in children presenting to the King Edward Hospital. GIl was the most prevalent genogroups with $100 \%$ of all positive cases belonging to GIl. Taking this into consideration with the lower sensitivity of the ELISA test, the RTqPCR would be more suitable to routinely testing stool specimens

\section{ID: 8521}

Category: Antimicrobial Resistance (SAASP)

Permission: Yes

Author: Maluleka, C

Author Institutes: N/A

E-mail Address: caroline.maluleka@nhls.ac.za

Country: South Africa

ABSTRACT TITLE: INVESTIGATION OF THE MOLECULAR PATTERNS OF CARBAPENEM-RESISTANT PSEUDOMONAS AERUGINOSA AND ACINETOBACTER BAUMANII, AND THE EFFICACY OF THE PHENOTYPIC DETECTION METHODS USED FOR DETECTING CARBAPENEM RE

\section{Introduction}

Carbapenem resistant Acinetobacter baumanii and Pseudomonas aeruginosa has been reported worldwide. These pathogens are the 
most common cause of nosocomial infections and they result in serious outbreaks in hospitals especially in ICU wards. Of concern is the multidrug resistance pattern of these pathogens resulting in challenges in their treatment. The resistant genes are plasmidmediated which could result in hospital outbreaks, a challenge for Infection control.

This study was done to compare the best method which can rapidly detect carbapenamase-producing organisms in Acinetobacter baumanii and Pseudomonas aeruginosa at the Dr George Mukhari Academic Hospital in 2015.

\section{Methods}

A total number of 35 Gram-negative isolates from National Health Laboratories Services (NHLS) at Dr George Mukhari Academic Hospital (DGMAH) and referral hospitals isolates with reduced susceptibility to carbapenems were collected from August to December 2015. Twenty-nine were Acinetobacter baumanii and 16 were Pseudomonas aeruginosa identified using the Vitek (BioMerieux). MICs for carbapenems were confirmed using an Etest. Resistant and intermediate isolates were further subjected to Rapidec Carba NP test. Conventional PCR was done to compare the sensitivity and specificity of the method. The PCR used targeted six common carbapenemase genes (BlaKPC, blaNDM, blaIMP, blaOXA-48, blaIMI, blaGES.)

\section{Results}

A total of $75.8 \%$ (22/29) Acinetobacter baumanii isolates were resistant and $3.4 \%$ were in the intermediate category for carbapenem. Twenty-one isolates (21/29) $72.4 \%$ were Rapidec Carba NP test positive. Rapidec Carba NP test had a sensitivity of $95 \%$ and a specificity of $100 \%$ for A. baumanii isolates. Eight (8/16) $50 \%$ Pseudomonas aeruginosa isolates had MIC ranging between $4 \mu \mathrm{g} / \mathrm{ml}$ to $32 \mu \mathrm{g} / \mathrm{ml}, 43.8 \%$ (7/16) were positive for Carba NP test and $56.3 \%$ (9/16) were positive for PCR.

\section{Conclusion}

Carba NP test is the most efficient phenotypic method for detecting carbapenamase-producing organisms, it is rapid and easy to perform. The circulating carbapenamase genes were blaGES for Acinetobacter baumanii and for Pseudomonas aeruginosa are blaVIM and blaKPC.

\section{ID: 8525}

Category: Antimicrobial Resistance (SAASP)

Permission: Yes

Author: Molaba, G

Author Institute: N/A

E-mail Address: mmadira1980@gmail.com

Country: South Africa

\author{
ABSTRACT TITLE: COMPARISON OF MINIMAL \\ INHIBITORY CONCENTRATION (MIC) VALUES PERFORMED \\ BY MICROSCAN WITH SENSITITRE PANELS FOR TESTING \\ OF ENTEROBACTERIACEAE
}

\section{Introduction}

Enterobacteriaceae are large group of Gram-negative, rodshaped bacteria which are the most frequently isolated from clinical specimens. They may account for up to $80 \%$ of clinically significant Gram-negative bacilli and up to $50 \%$ of all clinically significant bacteria. Enterobacteriaceae cause both nosocomial and community-acquired infections and are increasingly resistant to antimicrobial agents. Laboratory identification and susceptibility testing are essential for diagnostic and antimicrobial stewardship programmes. The aim of the study was to compare antimicrobial susceptibility testing results by the MicroScan ${ }^{\oplus}$ with Sensititre MICs panels for Enterobacteriaceae.

\section{Methods}

Total number of 49 isolates from August 2015 to December 2016 were obtained from patients with clinically diagnosed bloodstream infections randomly selected and used to determine the MIC. Results of MicroScan testing were extracted from the access database. The same isolates stored in -70 were then processed using Sensititre ${ }^{\circledast}$ $\mathrm{AIM}^{\mathrm{TM}}$ panels and read using Vizion ${ }^{\circledR}$ instrument.

\section{Results}

Total of 49 isolates, including 38 Klebsiella pneumoniae, 4 Enterobacter cloacae and Escherichia coli, 1 Klebsiella oxytoca, Klebsiella variicola and Serratia marcesens were processed. The following percentages of agreement was obtained for the antibiotics tested: ciprofloxacin $98 \%$, cefotaxime $96 \%$, ceftazidime $96 \%$, gentamicin $98 \%$, amikacin $98 \%$, ertapenem $76 \%$, imipenem $92 \%$ and tigecycline $100 \%$. No errors were obtained for ceftazidime, amikacin and tigecycline.

\section{Conclusion}

The two instruments compared well for most of the antibiotics. Only ertapenem had decreased agreement and cannot be used as screening antibiotic for carbapenemases-producing Enterobacteriaceae.

\section{ID: 8335}

Category: Infectious Diseases (IDSSA)

Permission: Yes

Co-Author 1: Quan, V

Co-Author 1 Institute: National Institute for Communicable Diseases

Co-Author 2: Simpson, GJ

Co-Author 2 Institute: Onderstepoort Faculty of Veterinary Science, University of Pretoria

Co-Author 3: van Rooyen, J

Co-Author 3 Institute: Faculty of Veterinary Science, University of Pretoria

Co-Author 4: Rossouw, J

Co-Author 4 Institute: National Institute for Communicable Diseases Co-Author 5: Weyer, J

Co-Author 5 Institute: National Institute for Communicable Diseases 
Co-Author 6: Frean, J

Co-Author 6 Institute: National Institute for Communicable Diseases

Co-Author 7: Blumberg, LH

Co-Author 7 Institute: National Institute for Communicable Diseases

E-mail Address: vanessaq@nicd.ac.za

Country: South Africa

\section{ABSTRACT TITLE: ZOONOTIC AETIOLOGIES IN FEBRILE ADULTS IN THE MNISI COMMUNITY, MPUMALANGA, SOUTH AFRICA}

\section{Introduction}

Zoonoses cause infectious diseases in humans who interact with livestock, domestic animals and vectors. A high prevalence of zoonotic infections was observed in a previous study at three public primary health clinics (PHCs) in Mnisi. Subsequently, a community health centre ( $\mathrm{CHC}$ ) in Mnisi was established as a sentinel site for the NICD surveillance programme which allows for seroprevalence monitoring of selected zoonotic diseases in an agro pastoral rural community.

\section{Methods}

From September 2014 to December 2016, consenting adult patients with fever $\left(>37.5^{\circ} \mathrm{C}\right) /$ history of fever on whom a malaria rapid test was done were enrolled from one $\mathrm{CHC}$ and a questionnaire administered. Acute and convalescent blood samples were collected for laboratory testing for leptospirosis, Q fever, bartonellosis, brucellosis, arboviruses and rickettsia.

\section{Results}

In total, 70 patients were enrolled: $46 \%$ (32/70) did not return for follow-up bloods. Median age was 34 years (IQR 26-46 years); $60 \%$ were female. Median duration of illness was two days (IQR $1-3$ days); $60 \%$ (40/67) received an antibiotic at the clinic and $11 \%(8 / 70)$ referred to the hospital. Twenty-four percent (17/70) of patients had no systemic symptoms, 59\% (31/53) presented with only one symptom: muscle pain $(67 \%)$ and respiratory symptoms (39\%) were most common. On laboratory testing, 79\% (55/70) of patients showed evidence of a recent or past infection/exposure for at least one of the zoonotic diseases: 63\% (42/67) for tick bite fever, 19\% (13/70) for Q fever, 4\% (3/70) for arboviruses, 1/70 for leptospirosis and zero for bartonellosis or brucellosis.

\section{Conclusion}

Compared to the previous study at three PHCs, fewer patients at this one $\mathrm{CHC}$, tested positive for $\mathrm{Q}$ fever (18.6\% vs $37.8 \%$; $p$-value 0.0110 ) and bartonellosis ( $0 \%$ vs $9.5 \%$, p-value 0.0358 ). Possible reasons for these differences may include: i) patients were enrolled from only one $\mathrm{CHC}$, ii) the demographic may be different, iii) there was a drought which could decrease vectors, and iv) fewer patients returned for follow-up blood samples.

\section{ID: 8404}

Category: Infectious Diseases (IDSSA)

Permission: Yes

Co-Author 1: Legenza, L

Co-Author 1 Institute: University of Wisconsin-Madison School of Pharmacy

Co-Author 2: Coetzee, R

Co-Author 2 Institute: University of the Western Cape School of Pharmacy

Co-Author 3: Barnett, $S$

Co-Author 3 Institute: University of Wisconsin-Madison School of Pharmacy

Co-Author 4: Rose, W

Co-Author 4 Institute: University of Wisconsin-Madison School of Pharmacy

Co-Author 5: Bianchini, $M$

Co-Author 5 Institute: University of Wisconsin-Madison School of Pharmacy

Co-Author 6: Safdar, N

Co-Author 6 Institute: University of Wisconsin School of Medicine and Public Health

E-mail Address: Legenza@wisc.edu

Country: South Africa and The United States of America

\section{ABSTRACT TITLE: CLOSTRIDIUM DIFFICILE INFECTION PATIENT CHARACTERISTICS AND ASSOCIATION WITH IN-HOSPITAL MORTALITY IN SOUTH AFRICA}

\section{Introduction}

Clostridium difficile infection (CDI) is a global health problem resulting in severe diarrhoea and increased morbidity and mortality. The South African population includes a high prevalence of HIV and tuberculosis. These patients often have prior healthcare exposures and high risk for CDI. CDI has not been well studied in this population. This study aimed to identify baseline patient CDI characteristics and associations with patient outcomes.

\section{Method}

A retrospective review of adult patients with diarrhoea and a CDI test result was conducted at four secondary level public sector hospitals in Cape Town, South Africa. Patient characteristics, CDI management, and patient outcomes were collected. Univariate, logistic regression, and survival analyses were performed.

\section{Result}

A total of 116 positive and 148 negative CDI test results were reviewed. In-hospital mortality was more common in the CDI positive group ( $28 \%$ vs. $8 \%, \mathrm{P}<0.0001$; hazard ratio $2.1,95 \%$ Cl 1.2-3.7). A logistic regression including CDI test result, prior hospitalisation (30-day and 90-day), critical care, tuberculosis, gender, multi-drug resistant tuberculosis, and hematochezia, found an independent risk of in-hospital mortality with a CDI positive test result $(\mathrm{OR} 4.4, \mathrm{P}=0.001)$. Clinically meaningful independent 
variables associated with mortality included comorbid tuberculosis (OR 2.2, $\mathrm{P}=0.035)$ and male gender $(\mathrm{OR} 2.5, \mathrm{P}=0.05)$. Tuberculosis was also significantly associated with a $\mathrm{CDI}$ positive test result $(P<0.05)$. Antibiotic exposure and hospitalisation in 30 days prior to $C D I$ test were significantly associated with a $C D I$ positive result $(P<0.05)$, but did not impact mortality.

\section{Conclusion}

Patients testing positive for CDI in South Africa have a significantly higher risk of mortality. Male patients are also at higher risk of inhospital mortality, but there was no gender association with CDI test result. Initial findings suggest tuberculosis comorbidity may be considered a risk factor for $\mathrm{CDI}$, in addition to known risk factors, prior antibiotic exposure and recent hospitalisation.

\section{ID: 8475}

Category: Infectious Diseases (IDSSA)

Permission: Yes

Co-Author 1: Moodley, S

Co-Author 1 Institute: Medical Microbiology and Infection Control, School of Laboratory Medicine and Medical Sciences, College of Health Sciences, University of KwaZulu-Natal

Co-Author 2: Mvubu, NE

Co-Author 2 Institute: School of Life Sciences, College of Agriculture, Engineering and Science, University of KwaZulu-Natal

Co-Author 3: Pillay, B

Co-Author 3 Institute: School of Life Sciences, College of Agriculture, Engineering and Science, University of KwaZulu-Natal

Co-Author 4: Pillay, M

Co-Author 4 Institute: Medical Microbiology and Infection Control, School of Laboratory Medicine and Medical Sciences, College of Health Sciences, University of KwaZulu-Natal

E-mail Address: suventha@gmail.com

Country: South Africa

\section{ABSTRACT TITLE: MYCOBACTERIUM TUBERCULOSIS HBHA AND MTP DELETION ELICITS UNIQUE CANONICAL PATHWAYS DURING EARLY INFECTION IN THP-1 DIFFERENTIATED MACROPHAGES}

\section{Introduction}

M. tuberculosis was responsible for an estimated 10.4 million new cases and 1.4 million deaths in 2015 (WHO, 2016). Understanding host gene regulation provides vital information about pathogenesis that possibly translates into novel therapeutic targets. This study utilised THP-1 human macrophages infected with a hbhA-mtp knockout mutant of Mycobacterium tuberculosis to assess the effect of hbhA gene deletion in the $\Delta$ mtp mutant on the host transcriptome.

\section{Methods}

In order to compare the transcriptome changes elicited by wild type (WT) and $\Delta$ hbhA-mtp infection at $4 \mathrm{hr}$ post-infection, RNA was extracted from THP-1 differentiated macrophage monolayers and sequenced using Hiseq 2500 platform (Illumina). Reads were trimmed using Trimmomatic and mapped to the Homo sapiens reference genome Hg19 (UCSC) using TopHat (2.1.0) and Bowtie2. Differential gene expression patterns were identified using cuffdiff. Changes in canonical pathways were analysed by Ingenuity Pathway Analysis.

\section{Results}

WT infection enriched the TREM1 signalling pathway to a greater degree than $\Delta$ hbhA-mtp infection in macrophages. $\Delta$ hbhA-mtp infection enriched the dendritic cell maturation pathways to a greater degree than WT infection. $\triangle \mathrm{hbhA-mtp} \mathrm{infection,} \mathrm{but} \mathrm{not} \mathrm{WT,}$ enriched Th1 and Th2 Activation, Th1, Th2, melatonin degradation, sumoylation, methylglyoxal degradation III, granzyme A signalling, PCP pathways.

\section{Conclusions}

The absence of HBHA and MTP proteins resulted in activation of host immune responses that are detrimental to the survival of M. tuberculosis, suggesting that these adhesins may assist the pathogen to evade the host immune responses during early infection.

\section{ID: 8501}

Category: Infectious Diseases (IDSSA)

Permission: Yes

Co-Author 1: Koekemoer, C

Co-Author 1 Institute: University of Pretoria

Co-Author 2: Ehlers, $M$

Co-Author 2 Institute: University of Pretoria

Co-Author 3: Kock, $M$

Co-Author 3 Institute: University of Pretoria

E-mail Address: chantalkoekemoer@yahoo.co.za

Country: South Africa

\section{ABSTRACT TITLE: PREVALENCE OF ANTIBIOTIC RESISTANCE AND VIRULENCE GENES OF ESCHERICHIA COLI O157: H7 ISOLATED FROM PORCINE IN GAUTENG, SOUTH AFRICA}

\section{Introduction}

Escherichia coli (E. coli) is an emerging pathogen, harboured in the gastrointestinal tract of mammals. Most $E$. coli strains are harmless, however, some $E$. coli strains harbour virulence factors which cause intestinal and extra-intestinal diseases in animals and humans. Limited information is available on the molecular characteristics and antibiotic resistance of $E$. coli $0157: \mathrm{H} 7$ isolates from pigs in South Africa. The study's aim was to identify presumptive $E$. coli 0157:H7 isolates obtained from slaughtered pigs from five Gauteng abattoirs and to determine the prevalence of selected virulence and antibiotic resistance genes. 


\section{Methods}

Isolates $(n=195)$ were collected from porcine rectal swabs. Methods used to confirm and identify presumptive E. coli 0157:H7 isolates are: ChromID ${ }^{\text {TM }}$ 0157:H7 (bioMérieux, France) agar, MALDITOF (Daltonics, USA) analysis and a Multiplex PCR (M-PCR) assay [detecting 0157 antigen ( $\mathrm{rfbE}$ ) andH7 flagella (fliC) genes]. M-PCR assays were performed to detect selected virulence [Shiga toxin 1 and 2 (Stx 1 and Stx 2), intimin (eae) and haemolysin (hlyA)] and antibiotic resistance [Sulfonamide (sul 1), Tetracycline (tet $A$ \& tetB), Chloramphenicol (cmlA), Trimethoprim (dhfr 1), Aminoglycoside (AadA)] genes.

\section{Results}

Isolates ( $\mathrm{n}=77$ ) were identified as $E$. coli. Six isolates showed prevalence of fliC and rfbE genes, confirming E. coli O157:H7. Results shown prevalence $33.3 \%$ (2/6), 83.3\% (5/6) and $100 \%$ of Stx 2, eae and hlyA virulence factors, respectively. No Stx 1 was detected. The tetA, dhfr 1 and sul 1 genes were detected in $50 \%(3 / 6)$ of the isolates. The tetB and $\mathrm{cml} A$ genes were detected in $66.7 \%(4 / 6)$ of isolates. All isolates were positive for the aadA gene.

\section{Conclusion}

Detection of virulence genes (stx2, eae and hlyA) presents potential health risks to close human contacts (abattoir workers) and consumers of undercooked meat products. Antibiotic resistant genes (tet $A$, tetB, sul1, aadA and dhfr1) detected, confer resistance to different antibiotic classes. Continuous surveillance for $E$. coli 0157:H7 is therefore important at the human-animal interface to prevent possible outbreaks.

\section{ID: 8526}

Category: Infectious Diseases (IDSSA)

Permission: Yes

Co-Author 1: Carrim, M

Co-Author 1 Institute: Centre for Respiratory Diseases and Meningitis, National Institute for Communicable Diseases of the National Health Laboratory Service

\section{Co-Author 2: Wolter, N}

Co-Author 2 Institute: Centre for Respiratory Diseases and Meningitis, National Institute for Communicable Diseases of the National Health Laboratory Service; School of Pathology, Faculty of Health Sciences, University of the Witwatersrand

Co-Author 3: du Plessis, M

Co-Author 3 Institute: Centre for Respiratory Diseases and Meningitis, National Institute for Communicable Diseases of the National Health Laboratory Service; School of Pathology, Faculty of Health Sciences, University of the Witwatersrand

Co-Author 4: Stewart, $R$

Co-Author 4 Institute: Infection Control Services, National Health Laboratory Service

Co-Author 5: de Gouveia, L

Co-Author 5 Institute: Centre for Respiratory Diseases and Meningitis, National Institute for Communicable Diseases of the
National Health Laboratory Service

Co-Author 6: von Gottberg, A

Co-Author 6 Institute: Centre for Respiratory Diseases and Meningitis, National Institute for Communicable Diseases of the National Health Laboratory Service; School of Pathology, Faculty of Health Sciences, University of the Witwatersrand

E-mail Address: maimunac@nicd.ac.za

Country: South Africa

\section{ABSTRACT TITLE: MOLECULAR EPIDEMIOLOGY OF LEGIONELLA PNEUMOPHILA IN SOUTH AFRICAN HOSPITALS, 2015-2016}

\section{Introduction}

Healthcare facilities globally are often identified as sources of Legionnaire's disease (LD) due to the combination of large, complex plumbing systems, and potential exposure to individuals with increased risk of infection. Globally, serogroup (SG) 1, sequence type (ST) 1 is the most common strain identified by sequencedbased typing (SBT). Due to limited data, we aimed to describe the molecular epidemiology of L. pneumophila isolated from hospitals in South Africa.

\section{Methods}

From March 2015 through April 2016, 77 Legionella spp. isolates were isolated from routine water samples or site investigations from 17 private and public hospitals, representing four of the nine provinces [Eastern Cape $(n=4)$, Western Cape $(n=4)$, Gauteng ( $n=8$ ) and Mpumalanga( $n=1)$ ] where there is an increased awareness for testing and notification of LD. Sequences of the 7 SBT genes were extracted from whole genome sequencing data, allele numbers and STs were assigned using the online L. pneumophila SBT database.

\section{Results}

Of the 77 isolates, 42 (55\%) were L. pneumophila SG1, 31 (40\%) were L. pneumophila SG2-14 and 4 (5\%) were other Legionella spp. Of all L. pneumophila isolates, we identified 4 known STs: ST1 (59\%; 43/73), ST421 (21\%; 15/73), ST87 (1\%; 1/73) and ST242 (1\%; 1/73). SG1 comprised ST1 (91\%; 30/33) and ST421 (9\%; 3/33) while SG214 comprised ST1 (50\%; 13/26), ST421 (42\%; 11/26), ST87 (4\%; $1 / 26)$ and ST242 (4\%; 1/26). We identified 13 isolates with novel STs identified at multiple hospitals. L. pneumophila SG1 ST1 was the most common strain, detected in 59\% (10/17) of hospitals. Three STs were identified in one Gauteng hospital [4/20 (20\%) ST1, 15/20 (75\%) ST421 and 1/20 (5\%) novel ST].

\section{Conclusions}

SG1 ST1 was the most common strain detected in the water systems of hospitals, as has been described previously. Amongst SG2-14 isolates, two additional STs (ST87 and ST242) were identified compared to SG1 isolates. STs that have not previously been described were identified. 


\section{ID: 8534}

Category: Infectious Diseases (IDSSA)

Permission: Yes

Co-Author 1: Ravhuhali, K

Co-Author 1 Institute: South African Field Epidemiology Training Programme

Co-Author 2: Reddy, C

Co-Author 2 Institute: South African Field Epidemiology Training Programme

Co-Author 3: Ramalwa, N

Co-Author 3 Institute: National Institute for Communicable Diseases

E-mail Address: ravhuhali.khuliso@yahoo.com

Country: South Africa

\section{ABSTRACT TITLE: MALARIA OUTBREAK INVESTIGATION - MOPANI AND VHEMBE DISTRICTS, LIMPOPO PROVINCE, SOUTH AFRICA, MAY 2017}

\section{Background}

Malaria remains a major public health challenge in Vhembe and Mopani districts, Limpopo province - two of the five malaria endemic districts in SA. On 2 May 2017, the two districts reported unusual increase of malaria cases. Investigations were conducted to establish existence, magnitude and cause of the outbreak, to recommend control measures and future prevention.

\section{Methods}

A cross-sectional investigation was conducted. Discussions with key stakeholders were held. Malaria case data from April to May 2017 were reviewed. A case was defined as a person with malaria confirmation by microscopy or a rapid diagnostic test (RDT) in health facilities of the two districts from April-May 2017.

\section{Results}

A total of 5662 cases, $55.3 \%$ in Mopani, were reported with a peak in May 2017. Investigation of likely origin revealed that 5574 (98.4\%) were local cases in both districts. Fifty-four malaria deaths were reported, the majority $42(77.8 \%)$ in the Mopani district. The median age of the patients who died was 44 years (IQR: $35-60$ ) with $0.95 \%$ case fatality rate (CFR). Indoor residual spraying (IRS) coverage was less than the WHO recommended $80 \%$ universal coverage for control. Environmental assessment revealed that the 2016/17 season recorded higher levels of rainfall compared to previous malaria seasons. Key stakeholder interviews revealed that the outbreak was associated with: late commencement of IRS due to late appointment of spray teams; reduced use of dichlorodiphenyltrichloroethane (DDT) replaced by pyrethroids (K-Othrine and deltamethrine); and stock outs of RDTs and antimalaria drugs in clinics.

\section{Conclusion}

The investigation established that the marked increase in malaria cases in the two districts was not a seasonal phenomenon but rather an outbreak. We recommended: an awareness programme through local radio stations on malaria prevention and treatment; an improved supply chain of RDTs and antimalarial drugs; adequate budget for procurement of DDT and advance appointment of IRS teams.

\section{ID: 8552}

Category: Infectious Diseases (IDSSA)

Permission: Yes

Co-Author 1: Makhado, N

Co-Author 1 Institute: Sefako Makgatho Health Sciences University

Co-Author 2: Maluleka, C

Co-Author 2 Institute: Sefako Mkgatho Health Sciences University

Co-Author 3: Nchabeleng, $M$

Co-Author 3 Institute: Sefako Makgatho Health Sciences University

E-mail Address: nmakhado@yahoo.com

Country: South Africa

ABSTRACT TITLE: CHARACTERISATION OF RIFAMPICIN AND ISONIAZID RESISTANCE OF MYCOBACTERIUM TUBERCULOSIS STRAINS FROM CLINICAL SPECIMENS SENT TO DR GEORGE MUKHARI TERTIARY LABORATORY IN SOUTH AFRICA

\section{Introduction}

Tuberculosis (TB) continues to be a global threat. A series of molecular characterisation of the organism have since been developed and introduced, which include the MTBDRplus assay that detects Mycobacterium tuberculosis (MTB) and susceptibility to rifampicin (RIF) and isoniazid (INH); and GeneXpert MTB/RIF assay that detects MTB and susceptibility to only RIF. This study was conducted to characterise the drug resistant patterns of M. tuberculosis strains.

\section{Methods}

This was a retrospective data analysis of MTB from the Laboratory Information System (LIS) TrackCare from 01 January 2016 to 31 December 2016. A total of 11829 results were retrieved from specimens collected from different body sites sent to the National Health Laboratory Service, Dr George Mukhari (NHLS-DGM) Tertiary Laboratory, Medical Microbiology Department, during the study period for routine TB tests including microscopy, culture, and drug susceptibility testing using the MTBDRplus genotypic assays.

\section{Results}

Of the 11829 clinical results retrieved, $6591(56 \%)$ were MTB positive. In total, 5356 (81,3\%) were fully sensitive and 1235 (18,7\%) were resistant to either RIF, INH or both drugs. A total of $415(33,6 \%)$ were multidrug-resistant (MDR) TB; 422 (34.2\%) were RIF monoresistant; 398 (32,1\%) were INH monoresistant; overall, 510 (61.3\%), RIF resistance was due to MUT3 in the rpoB gene. The prevalent INH resistance was due to katG gene mutation (MUT1) $(49,9 \%)$. 


\section{Conclusion}

This study shows that the most prevalent mutations in rpoB gene for RIF are caused by mutation in the MUT3 and MUT1 in the katG gene for INH.

\section{ID: 8321}

Category: Antimicrobial Resistance (SAASP)

Permission: Yes

Co-Author 1: Esmaio, $M$

Co-Author 1 Institute: Microbial Endogenous Infections Studies Research Laboratories, Department of Medical Biosciences, University of the Western Cape

Co-Author 2: Abrantes, P

Co-Author 2 Institute: Microbial Endogenous Infections Studies Research Laboratories, Department of Medical Biosciences, University of the Western Cape

Co-Author 3: Africa, C

Co-Author 3 Institute: Microbial Endogenous Infections Studies Research Laboratories, Department of Medical Biosciences, University of the Western Cape

E-mail Address: esmaio79@gmail.com

Country: South Africa

\section{ABSTRACT TITLE: CANDIDA SPECIES CARRIAGE IN DIABETIC PATIENTS IN MISRATA, LIBYA}

\section{Introduction}

There is a paucity of studies describing the prevalence and antimicrobial profiles of Candida in Libya. Limited treatment choices in the antifungal armamentarium in public healthcare settings in the rest of Africa require a study of the prevalence and susceptibility of Candida species in Libya, where antifungals are not regularly distributed in public healthcare settings.

\section{Methods}

In this study, 170 diabetic mellitus type 2 (T2DM) patients were examined for Candida carriage in the oral mucosa, using differential/ Fluka and Oxoid chromogenic media and API 32 ID C biochemical testing. Fluconazole susceptibility was investigated by disc diffusion on YNBG agar. Isolates were graded as susceptible, intermediate or resistance according to their inhibition zone measurements and microcolony scores.

\section{Results}

Thirteen species were identified from 182 isolates with a frequency of 69 C. albicans, 42 C. dubliniensis, 26 C.humicola, 20 C. glabrata, 5 isolates of $C$. krusei, C. tropicalis and C. kefyr respectively, 4 C.sake, 2C. parapsilopsis, 2 C. magnoliae and 1 isolate each of C. guilliermondii, C. globosa and C. membranifaciens. Although largely susceptible to fluconazole, C. albicans, C. dubliniensis, C. humicola and C. sake demonstrated an emerging resistance with intermediate to total resistance observed in all the other species except for $C$. magnolia and C. globosa which were both susceptible to fluconazole.

\section{Conclusion}

Early recognition and treatment of rare or resistant species which may be contributing to patient morbidity and mortality in Libya is imperative.

This study was funded by the Libyan government.

\section{ID: 8393}

Category: Antimicrobial Resistance (SAASP)

Permission: Yes

Co-Author 1: Samie, A

Co-Author 1 Institute: University of Venda

Co-Author 2: Beke, A

Co-Author 2 Institute: University of Pretoria

E-mail Address: samieamidou56@gmail.com

Country: South Africa

\section{ABSTRACT TITLE: PERCEPTIONS AND PRACTICE OF INFECTION CONTROL AMONG MEDICAL STAFF AT FOUR MAIN HOSPITALS IN THE VHEMBE DISTRICT}

\section{Introduction}

Recent studies have shown that nosocomial infections are often spread through devices used by healthcare professionals. The present study assessed the perceptions and practice of infection control (IC) by hospital staff in Vhembe District and evaluated the contamination of stethoscopes and cell phones.

\section{Methods}

In a cross-sectional study, questionnaires were administered to physicians and nurses. Stethoscopes and cell phones were swabbed for the isolation of microorganisms.

\section{Results}

A total of 178 participants aged between 23 and 65 years with a mean of $42.29 \pm 9.76$ years were recruited in the study. About $46 \%$ of staff cleaned their stethoscopes sometimes and handwashing was practiced by $52 \%$ of them. They were largely ignorant of other IC strategies such as antibiotic stewardship, surveillance or vaccinations. Many participants indicated that further education should be provided and more resources allocated for IC. About $88 \%$ of cell phones and $80 \%$ of stethoscopes were contaminated and yeast was the most commonly isolated microorganisms while Staphylococcus spp were isolated from $27 \%$ of the stethoscopes.

\section{Conclusion}

The present study showed that the practice of infection control by hospital staff is still poor and there is need for further effort and education to hospital employees in order to empower them on infection control strategies. Stethoscopes and cell phones are carriers of pathogens and their cleanliness should be emphasised among healthcare workers. 


\section{ID: 8452}

Category: Infectious Diseases (IDSSA)

Permission: Yes

Authors: Moodley, K; Govind, CN; Peer, AK; Prinsloo, B; Wojno, $\mathrm{JM}$; Seetharam, S

Author institutions/affiliations: Lancet Laboratories; Lancet Laboratories; Lancet Laboratories; Lancet Laboratories; Lancet Laboratories; Lancet Laboratories

E-mail Address: krishnee.moodley@lancet.co.za

Country: South Africa

\section{ABSTRACT TITLE: EMERGENCE OF CANDIDA AURIS IN SOUTH AFRICAN PRIVATE SECTOR HOSPITALS}

\section{Introduction}

Candida auris, first described in 2009 after isolation from the external ear canal of a patient in Japan has emerged as a global pathogen, including in South Africa. Phenotypically, it resembles Candida haemulonii and until recently, required molecular methods for identification. Currently, both matrix-assisted laser desorption/ ionisation-time of flight (MALDI-TOF) mass spectrometry and the Vitek-2 systems can reliably identify this species. This organism has been associated with hospital outbreaks and multidrug resistance (MDR; resistance to two or more antifungal classes).

We report on the emergence of Candida auris bloodstream infections in a private healthcare setting in South Africa and describe the susceptibility patterns of this emerging pathogen.

\section{Methods}

All Candida species identified as C. auris or C. haemulonii from blood cultures from January 2015-June 2017 were analysed. Species identification was made using the MS or Vitek systems (bioMerieux, Marcy l'Etoile, France).

There are no agreed-upon interpretive breakpoints for $C$. auris for antifungal agents; however, conservative breakpoints, developed for other Candida species, have been applied to C. auris. The Vitek (bioMerieux, Marcy l'Etoile, France) or Sensititre YeastOne ${ }^{\oplus}$ (Trek Diagnostic Systems Ltd) were used to perform sensitivity testing.

\section{Results}

Of 1625 Candida species blood culture isolates from January 2015 through to June 2017, 14\% (223/1 625) were Candida auris/ haemulonii, of which $98 \%$ (219/223) were from the Gauteng province. The age range was 0-94 years (median 53 years), 57\% $(126 / 223)$ being $>50$ years old. Fifty-nine percent of cases were in ICU/high care wards. Susceptibility to the antifungals: $97 \%$ (216/223), 2\% (4/223), 86\% (31/223) and 98\% (218/223) were susceptible to amphotericin $B$, fluconazole, voriconazole and the echinocandins, respectively.

\section{Conclusion}

Candida auris, the second most frequent cause of non-albicans candidaemia in the private sector in South Africa, exhibited regional variation and azole-resistance. Further studies will be useful in monitoring the evolution of the susceptibility patterns of Candida auris bacteraemia in South Africa.

\section{ID: 8499}

Category: Infectious Diseases (IDSSA)

Permission: Yes

Authors: Steyn, J; Ehlers, M; Kock, M; Meidany, P; Said, M

Author institutions/affiliations: University of Pretoria; University of Pretoria/NHLS; University of Pretoria/NHLS; University of Pretoria; University of Pretoria/NHLS

E-mail Address: u15267882@tuks.co.za

Country: South Africa

\section{ABSTRACT TITLE: GENETIC RELATEDNESS OF CLINICAL CLOSTRIDIUM DIFFICILE ISOLATES IN THE PRETORIA REGION}

\section{Introduction}

Clostridium difficile is the leading cause of healthcare associated diarrhoea globally. The spectrum of disease may range from mild diarrhoea to severe colitis. The aim of this study was to determine the genetic relatedness of clinical C. difficile isolates in Pretoria.

\section{Methods}

A total of 97 stool specimens submitted to the Diagnostic Division of the Department of Medical Microbiology/National Health Laboratory Service were collected from June 2016 to April 2017 after routine diagnosis. Stool specimens were collected which tested positive for toxin A/B by the C Diff Quik Chek Complete ${ }^{\circledR}$ assay or positive for the tcdB gene by the Cepheid GeneXpert $C$. difficile ${ }^{\circledast}$ real-time PCR assay by a two-step algorithm. The $C$. difficile isolates were treated with the alcohol shock method and cultured anaerobically at $37^{\circ} \mathrm{C}$ for $48 \mathrm{~h}$ to $72 \mathrm{~h}$. The toxin genes were detected using M-PCR assays. Molecular typing was performed on the isolates using PCR ribotyping. Patient clinical data was collected by analysis of patient records and consulting the attending physician.

\section{Results}

The patient demographics were: $64 \%$ (62/97) female and $36 \%$ (35/97) male with a mean age of 40 years. Approximately $49 \%$ of patients are aged between 30 and 49 years, which is highly significant as CDI has historically been associated with patients aged $\geq 65$. The most commonly used antibiotics were piperacillintazobactam (20\%) followed by amoxicillin (12.37\%). Toxin A (tcdA) and toxin $B(t c d B)$ was detected in all isolates, however the binary toxin was not detected.

\section{Conclusion}

Preliminary PCR ribotyping data show genetic relatedness between the isolates. The high prevalence of HIV among the patients contributes significantly to increased hospitalisation and exposure to antimicrobials which increases the risk for acquiring CDI. Surveillance of the circulating toxin-positive $C$. difficile isolates is important to prevent outbreaks of CDI. 


\section{ID: 8540}

Category: Infectious Diseases (IDSSA)

Permission: Yes

Co-Author 1: Yousefi, F

Co-Author 1 Institute: Health Research Institute, Infectious and Tropical Diseases Research Center, Ahvaz Jundishapur University of Medical Sciences

Co-Author 2: Mohammadi, MJ

Co-Author 2 Institute: Student Research Committee, Department of Environmental Health Engineering, School of Public Health and Environmental Technologies Research Center, Ahvaz Jundishapur University of Medical Sciences

Co-Author 3: Kamali, A

Co-Author 3 Institute: Ahvaz Jundishapur University of Medical Sciences

Co-Author 4: Salmanzadeh, S

Co-Author 4 Institute: Health Research Institute, Infectious and Tropical Diseases Research Center, Ahvaz Jundishapur University of Medical Sciences

E-mail Address: drfayo@gmail.com

Country: Iran

\section{ABSTRACT TITLE: HYDATID CYST DISEASE IN KHUZESTAN, IRAN, DURING 2000-2015}

\section{Introduction}

Hydatid cyst disease (HCD) is a well-known parasitic disease globally. It develops in humans after ingestion of Echinococcus granulosus eggs. In order to better prevent and control HCD, it is crucial to identify the epidemiologic aspects of this parasitic infection. The current study aimed to evaluate the epidemiology and features of $\mathrm{HCD}$ in a livestock-raising area in Khuzestan, southwest of Iran.

\section{Methods}

The present study was a descriptive-analytical study conducted on 360 patients from different areas of Khuzestan Province, southwest of Iran, with a diagnosis of HCD between 2000 and 2015, using their records. Demographic data, clinical features, and radiological data were collected. Data were summarised and analysed using descriptive and analytical statistical methods, respectively.

\section{Results}

$56.1 \%$ of the patients were female. The mean age of the patients was $37.36 \pm 15.2$ years. Most patients were in the over-50-yearsold age group [103 (28.6\%)], and the less-than-10-years-old age group had the lowest number [19 (5.3\%)]. Most of the cysts were detected in the liver [234 (65\%)]. Regarding the finding, there was no statistically significant association between sex and residing area, animal contact, and the number of the cysts $(p=0.12,0.36$, and 0.95 , respectively); however, a significant association was found between sex and the body organ involved ( $p=0.007)$, so that liver involvement was mostly detected in females (79.9\%), while involvement of the lung was mostly found in males (66.4\%). No statistically significant association was found between age and the number of the cysts or the body organ involvement $(p=0.35$ and 0.61 , respectively).

\section{Conclusion}

Our study showed that HCD could be surprisingly common in apparently low-risk populations, such as those living in urban areas or without direct contact with dogs and farm animals. So, educating the community about the most common modes of acquisition could be helpful for the control and prevention of this disease.

\section{ID: 8543}

Category: Infectious Diseases (IDSSA)

Permission: Yes

Co-Author 1: Souda, S

Co-Author 1 Institute: University of Botswana

Co-Author 2: George, $S$

Co-Author 2 Institute: Botswana Harvard AIDS Institute Partnership

Co-Author 3: Mannathoko, N

Co-Author 3 Institute: University of Botswana

Co-Author 4: Mwita, J

Co-Author 4 Institute: University of Botswana

Co-Author 5: Moyo, S

Co-Author 5 Institute: Botswana Harvard AIDS Institute Partnership Co-Author 6: Gaseitsiwe, $S$

Co-Author 6 Institute: Botswana Harvard AIDS Institute Partnership

E-mail Address: soudas@ub.ac.bw

Country: Botswana

\section{ABSTRACT TITLE: SEROPREVALENCE OF HEPATITIS E VIRUS INFECTION IN PATIENTS WITH LIVER DISEASE IN A REFERRAL HOSPITAL IN BOTSWANA}

\section{Introduction}

This study reports the seroprevalence of Hepatitis E Virus (HEV) infection in patients presenting to a referral hospital in Botswana.

\section{Methods}

A cross-sectional analysis of HEV infections among 331 patients presenting with clinical features of jaundice, elevated liver function tests, or a previous history of liver disease, was conducted at Princess Marina Hospital, Botswana from February 2015 to July 2016. Serum was screened for the presence of antibody to HEV IgM and $\mathrm{lgG}$, using enzyme linked immunosorbant assay techniques (ELISA). Risk factors for HEV infection were assessed using logistic regression techniques(STATA version 13.1)

\section{Results}

Of the 331 participants, antibody to HEV was positive in $9.7 \%$ ( $n=32$ ). Anti HEV IgM was positive only in one $(0.30 \%)$ of the 331 samples. Thirty-one (9.37\%) samples were positive for anti HEV IgG. HEV antibodies were detected in 17 (53\%) males and the median 
age was 45 years (Q1:36, Q3:56). Seven (4.6\%) patients with HEV positive serology were also HIV positive. Fifteen (48.4\%) HBsAg positive samples were also found to be anti HEV IgG positive. HCV confection was reported in 2 (6.5\%) samples. Associated symptoms included nausea and loss of appetite. Risk factors associated with HEV infection in this study were lack of proper hand washing, alcohol consumption, and HIV infection. People on HAART and sexually active people were also at risk of getting HEV infection. Low serum bilirubin and serum alkaline phosphatase were associated with HEV infection.

\section{Conclusion}

Prevalence of HEV infection is $9.7 \%$ in Botswana among patients with liver disease. This is the first study reporting the seroprevalence of HEV infection in Botswana. We recommend a routine hepatitis profile screening of all patients with clinical manifestations of liver disease as HEV infection can cause fulminant hepatitis.

\section{ID: 8580}

Category: Infectious Diseases (IDSSA)

Permission: Yes

Co-Author 1: Makhado, N

Co-Author 1 Institute: National Health Laboratory Service, Sefako Makgatho Health Sciences University

Co-Author 2: Nchabeleng, $M$

Co-Author 2 Institute: National Health Laboratory Service, Sefako Makgatho Health Sciences University

E-mail Address: nmakhado@yahoo.com

Country: South Africa

\section{ABSTRACT TITLE: THE INCIDENCE OF DISCORDANT RIFAMPICIN SUSCEPTIBILITY RESULTS BETWEEN GENOTYPIC AND PHENOTYPIC METHODS IN MYCOBACTERIUM TUBERCULOSIS COMPLEX ISOLATES AT DR GEORGE MUKHARI HOSPITAL TERTIARY LABORATORY}

\section{Introduction}

Drug resistant tuberculosis increasingly compromises the global success of tuberculosis treatment. Rapid and accurate detection of Mycobacterium tuberculosis and drug susceptibility testing to rifampicin and isoniazid is the first requirement to treat these patients correctly, which will in turn limit transmission of drug resistant strains. Frequent discordant results between routinely used phenotypic and genotypic drug susceptibility testing methods at the National Health Laboratory service - Dr George Mukhari Tertiary Laboratory, are continuously observed, especially with rifampicin posing challenges and delay in tailoring correct treatment for patient management. This study aimed to determine the incidence of discordant rifampicin susceptibility results between the genotypic and phenotypic drug susceptibility testing methods.

\section{Methods}

From June 2013 to December 2015, we consecutively collected 50 Mycobacterium tuberculosis rifampicin discordant samples with GenoType MTBDRplus and MGIT 960 phenotypic drug susceptibility testing. The genomic DNA of the discordant results was extracted and sent to the Inqaba Biotech Pty (Ltd) for the rpoB gene sequencing.

\section{Results}

Of the 50 samples in which the rpoBgene sequencing was done, rpoB gene sequencing yielded $41 / 50(82.0 \%)$ interpretable results. Of these, 39/41 (95.1\%) patients were co-infected with HIV. There were more females $21 / 41(51.2 \%)$ than males $20 / 41$ (48.8\%). Of the 41 resulted samples, $2 / 41(4.8 \%)$ yielded no rpoB mutation. The most prevalent mutation was Ser531Leu 12/40 (29.2\%) samples, followed by His526Leu 7/41 (17.0\%) samples; His526Asp 6/41 (14.6\%) samples; Leu511Pro 5/41 (12.1\%) samples; His526Try 5/41 (9.7\%) samples, and both Asp516Val as well as Asg218Hist in 2/41 (4.8\%) samples.

\section{Conclusion}

It is important to detect rpoB gene mutations to accurately and promptly diagnose drug-resistant strains to limit the delays in patient management. This study shows that DNA sequencing is the best suited for evaluating suspected drug-resistant Mycobacterium tuberculosis isolates with discordant results for phenotypic susceptibility and rapid molecular testing.

\section{ID: 8379}

Category: Antimicrobial Resistance (SAASP)

Permission:Yes

Co-Author 1: Said, M

Co-Author 1 Institute: University of Pretoria

Co-Author 2: Mbelle, N

Co-Author 2 Institute: University of Pretoria

E-mail Address: msaid@mweb.co.za

Country: South Africa

ABSTRACT TITLE: VERIFICATION OF THE HAIN MTBDRSL AND BD MGIT PHENOTYPIC DRUG SUSCEPTIBILITY TESTING (DST) FOR TUBERCULOSIS AT THE TSHWANE ACADEMIC LABORATORY (TAD)

\section{Introduction}

Drug resistant TB (DR-TB) is associated with higher morbidity and mortality than drug sensitive TB. Rapid detection of these strains is important for appropriate treatment as well as prevention of further transmission of these strains in the community. A tertiary academic microbiology laboratory such as TAD should be able to perform DST on these strains routinely. The aim of this study was to verify the MTBDRsI assay (LPA, Hain Lifescience, Nehren, Germany) and the MGIT (Becton, Dickinson and Company, USA) phenotypic DST assay. 


\section{Methods}

Our laboratory received a proficiency panel of twenty TB strains with varying drug susceptibility to the second line agents from the TB Reference Laboratory at the National Institute of Communicable Diseases (NICD, Sandringham, Johannesburg, South Africa). These strains were tested using the MTBDRsl assay (Version 2) and the MGIT phenotypic susceptibility testing using five drugs with concentrations of: amikacin $(1 \mu \mathrm{g} / \mathrm{ml})$, capreomycin $(2.5 \mu \mathrm{g} / \mathrm{ml})$, kanamycin $(2.5 \mu \mathrm{g} / \mathrm{ml})$, moxifloxacin $(0.5 \mu \mathrm{g} / \mathrm{ml}$ and $2.0 \mu \mathrm{g} / \mathrm{ml})$ and ofloxacin $(2.0 \mu \mathrm{g} / \mathrm{ml})$

\section{Results}

The TAD laboratory received a score of $95 \%$ for DST using the MTBDRsI assay. Nineteen strains were available to compare MTBDRsl and the BD MGIT phenotypic DST assay. There was one discrepant result for fluoroquinolone DST, that tested resistant with the MTBDRsl assay and sensitive with the phenotypic DST. This did not change on repeat testing. For aminoglycoside DST, there were no discrepancies observed between the two methods.

\section{Conclusion}

The TAD laboratory was found to be proficient in the use of the MTBDRsl assay. When the MTBDRsl assay was compared to the BD MGIT phenotypic susceptibility result, it had a sensitivity and specificity of $100 \%$ for aminoglycoside susceptibility and a sensitivity of $94 \%$ and a specificity of $100 \%$ for fluoroquinolone susceptibility. The TAD laboratory has subsequently implemented second-line testing for $\mathrm{TB}$ as a routine.

\section{ID: 8491}

Category: Antimicrobial Resistance (SAASP)

Permission: Yes

Co-Author 1: Anyanwu, RC

Co-Author 1 Institute: University of Port Harcourt/Mellow Works Ltd/STMRL

Co-Author 2: Okafor, DH

Co-Author 2 Institute: St Martha Research Laboratories/Mellow Works Ltd

E-mail Address: drreginald@mellowworks.com.ng

Country: Nigeria

\section{ABSTRACT TITLE: PREVALENCE AND INTENSITY OF SEXUALLY TRANSMITTED DISEASES (SASPID) IN LAGOS METROPOLITAN LAGOS STATE, NIGERIA (A CASE STUDY OF HIGHEST POPULATED COMMERCIAL SEX WORKERS (ENTRE)}

\section{Introduction}

The spread of sexually transmitted diseases is growing at an alarming rate through commercial sex workers. A survey carried out in one of the hotels considered as the highest population for commercial sex workers in Lagos, Nigeria reveals that $70 \%$ commercial sex workers were infected with different severities of diseases ranging from HIV, Hepatitis B, N. gonorrhea, Staphyloccocus aureus, Candida albicans, etc. $20 \%$ accounted for microscopically low pus cell count levels which could not yield significant growth after 48 hours of incubation in the laboratory. However, $10 \%$ indicated no record of any form of sexually transmitted diseases.

\section{Case}

In addition, $70 \%$ of the heavily infected cases of the study suggest that three-quarters accepted treatment and counselling while onequarter refused to submit themselves for medical treatment and counselling as a result of fear of exposure to the law enforcement agencies.

Three-quarters of the $20 \%$ lower cases accepted counselling while $2 / 5$ declined. All the patients with no record of sexually transmitted diseases accepted counselling and do not have any intent in quitting prostitution.

\section{Discussion}

The study reveals that the contributory factor to the prevalence and intensity are poverty (41\%), unemployment (35\%), education (10\%), government enforcement $(9.5 \%)$, refusal of male counterpart to apply contraceptive e.g. condom (4.5\%) and are responsible for the prevalence and intensity of sexually transmitted diseases among commercial sex workers.

Finally, recommendations were made on how to address these causes for a lasting solution. Three-hundred-and-fifteen commercial sex workers participated in the study.

\section{ID: 8493}

Category: Infectious Diseases (IDSSA)

Permission: Yes

Co-Author 1: Modipane, L

Co-Author 1 Institute: University of Pretoria

Co-Author 2: Reva, 0

Co-Author 2 Institute: University of Pretoria

Co-Author 3: Antiabong, JF

Co-Author 3 Institute: Centre for Infectious Diseases Research and Experimental Therapeutics, Baylor Research Institute

Co-Author 4: Magazi, BT

Co-Author 4 Institute: Merck Sharp and Dohme

Co-Author 5: Mbelle, NM

Co-Author 5 Institute: University of Pretoria

E-mail Address: leeprianca92@gmail.com

Country: South Africa

ABSTRACT TITLE: GENETIC DIVERSITY OF MYCOBACTERIUM BOVIS BCG STRAINS; CLINICAL AND VACCINE 


\section{Introduction}

Mycobacterium tuberculosis BCG is an attenuated strain of $M$. bovis used to vaccinate infants against disseminated tuberculosis. Attenuation of the vaccine resulted from mutation of the wildtype M. bovis. While protection from disseminated tuberculosis was shown in infants, complications following vaccination have been reported. Several BCG sub-strains now exist following passaging of the original Pasteur strain for vaccine manufacture. What has not been elucidated is whether these differences have any bearing on the risk for complication with each sub-strain.

The aim of this study was to identify genetic variation between the invasive BCG and non-invasive BCG reference strains.

\section{Methods}

In this study five clinical (M. bovis BCG Danish) isolates were studied. The M. bovis BCG Moscow vaccine was used as reference. Nitrate reduction test and pyrazinamide drug susceptibility tests were performed to confirm the isolates. The DNA was sequenced using the Illumina Miseq platform. Data was analysed using CLC Genomics workbench7 and Mauve 2.3.1; the sequences were compared with reference genome, $M$. bovis BCG Copenhagen (Danish).

\section{Results}

The biochemical assays confirmed that the clinical and the reference strains were $M$. bovis. Deletion of the RD1 region, confirmed the isolates as BCG. Deletions, insertions and single nucleotide variation were identified on the target isolates sequences in reference to the M. bovis BCG Copenhagen genome; the target isolates could have mutated.

\section{Conclusion}

Variation between the clinical and reference $M$. bovis BCG genome was confirmed. This could have contributed to the vaccination complications. Further studies are required to identify specific gene mutations that may contribute to dissemination in immunocompromised.

\section{ID: 8550}

Category: Infectious Diseases (IDSSA)

Permission: Yes

Co-Author 1: Souda, S

Co-Author 1 Institute: University of Botswana

Co-Author 2: Anderson, $M$

Co-Author 2 Institute: Botswana Harvard AIDS Institute Partnership Co-Author 3: George, $S$

Co-Author 3 Institute: University of Botswana

Co-Author 4: Mannathoko, N

Co-Author 4 Institute: University of Botswana

Co-Author 5: Mwita, J

Co-Author 5 Institute: University of Botswana

Co-Author 6: Moyo, S
Co-Author 6 Institute: Botswana Harvard AIDS Institute Partnership

Co-Author 7: Gaseitsiwe, S

Co-Author 7 Institute: Botswana Harvard AIDS Institute Partnership

E-mail Address: soudas@ub.ac.bw

Country: Botswana

ABSTRACT TITLE: PREVALENCE AND RISK FACTORS OF HEPATITIS B VIRUS INFECTIONS AMONG PATIENTS WITH LIVER DISEASE IN A PUBLIC HOSPITAL IN BOTSWANA

\section{Introduction}

Understanding the dynamic nature of chronic HBV infection is essential for management of HBV carriers.

\section{Methods}

A cross sectional study conducted among 331 patients presenting with jaundice, elevated liver function tests, or previous history of liver disease, attending Princess Marina Hospital, Gaborone, Botswana (February 2015 to July 2016). Serum screened for presence of Hepatitis B surface antigen ( $\mathrm{HBsAg}$ ), core antibody (anti$\mathrm{HBC}$ ), surface antibody (anti-HBs), Hepatitis B e antigen ( $\mathrm{HBeAg}$ ) and antibody to Hepatitis $B$ e antigen (anti-HBe) using enzyme linked immunosorbent assay techniques (ELISA). Risk factors assessed using logistic regression techniques.

\section{Results}

One-hundred-and-eighty-nine (57\%) of the 331 participants were females, with median age of 40 years (Q1, Q3: 31-53.5). Serological profiles show: $\mathrm{HBsAg}$ positive in $52.57 \%(95 \% \mathrm{Cl} 0.47-0.58)$; anti$\mathrm{HBC}$ in $56.5 \%$ (95\% Cl 0.51-0.61); HBeAg $8 \%(95 \% \mathrm{Cl} 0.05-0.11)$ and anti-HBe $34.74 \%$ (95\% Cl 0.22-0.31). Antibodies to HBsAg detected in 115 (34\%, 95\% Cl 0.29-0.40) samples. Using serological profiles, participants were classified into acute infection or with chronic active hepatitis (based on the presence of HBeAg ( $n=40,12.8 \%$ ); chronic hepatitis B patients ( $n=47,14.2 \%$ ); presence of immunity due to natural infection ( $n=53 ; 16 \%)$; probably hepatitis $B$ infection with anti-HBs below level of detection $(n=4 ; 1.21 \%)$. Anti-HBsAg alone indicating prior vaccination or infection ( $n=46,13.9 \%$ ); isolated anti-HBc detected in 22 (6.65\%) probably due to resolved infection, low level chronic infection or false positive implying they are susceptible. Fifty-two (15.7\%) patients were negative for all the serological markers tested. Mean alanine aminotransferase (ALT), aspartate aminotransferase (AST), serum bilirubin, alkaline phosphatase values were 230.8 (SD 410.58), 311.98 (SD 511.53), 83.01 (SD 7.47), and 287.55 (SD 395) respectively. All health professionals in the study were HBsAg positive.

\section{Conclusion}

Half of the patients with jaundice, history of jaundice or increased aminotransferase levels have hepatitis B infection. Screening for HBV infection and early management is advised. Health professionals need to be vaccinated compulsorily and anti-HBs titre evaluated. 


\section{ID: 8561}

Category: Infectious Diseases (IDSSA)

Permission: Yes

Author: Govender, V

Author Institute: Medical Microbiology and Infection Control, School of Laboratory Medicine and Medical Sciences, College of Health Sciences, University of KwaZulu-Natal

E-mail Address: shalomgovender@gmail.com

Country: South Africa

\section{ABSTRACT TITLE: MYCOBACTERIUM TUBERCULOSIS HEPARIN BINDING HAEMAGGLUTININ ADHESIN (HBHA) AND CURLI PILI (MTP) ARE ESSENTIAL FOR IN VITRO GROWTH, BUT NOT VIABILITY AND BIOFILM PRODUCTION}

\section{Introduction}

M. tuberculosis (M.tb) gene knockout mutants generated by phagemediated genetic approaches allow for the extensive study of specific encoded proteins and their role in persistence and virulence. Mycobacteria display multiple proteins with primary adhesin function that interact with host cells. The major adhesin is the heparin-binding haemagglutinin adhesin (HBHA), that facilitates dissemination of the pathogen. M.tb also produces pili proteins (MTP) during pathogenesis. This study proposed to establish the contribution of HBHA and MTP, in the growth, viability and biofilm production of M.tb.

\section{Methods}

A double mutant strain deficient in the adhesin molecules encoded by genes Rv3312A (mtp) and Rv0475 (hbhA) was generated by phage-mediated specialised transduction, followed by complementation of both the double and single mutants with episomal plasmids expressing the deleted genes. The phenotypic contribution of these molecules was assessed in vitro by Optical Density (OD 600) readings, Colony Forming Unit (CFU) counts, resazurin microplate assay (REMA) and crystal violet biofilm quantification.

\section{Results}

Growth rates varied among the wildtype, single and double mutant strains. However, no significant differences in the viability were observed among these strains. Biofilm production was similar between wildtype and mutant strains, alluding to possible compensatory gene regulation mechanisms in the absence of functional hbhA and mtp transcripts.

\section{Conclusion}

These findings demonstrate the importance of the two major adhesins in functioning together to facilitate the growth of M. tuberculosis. This is suggestive of their potential as biomarkers for drug or vaccine development.

\section{ID: 8678}

Category: Sexually Transmitted Diseases (STDSSA)
Permission: Yes

Author: Radebe, $\mathrm{F}$

Author Institute: National Institute for Communicable Diseases

E-mail Address: Fransr@nicd.ac.za

Country: South Africa

\section{ABSTRACT TITLE: THE ROLE AND IMPACT OF SEXUALLY TRANSMITTED INFECTIONS (STIS) SURVEILLANCE IN SOUTH AFRICA (2004-2016) - WHERE ARE WE NOW?}

\section{Introduction}

Since 2004 in South Africa, several successful attempts for comprehensive STI Surveillance were initiated: a) National microbiological surveillance in all provinces between 2004-2014 (syndromic STI aetiologies and antimicrobial resistance to Neisseria gonorrhoeae); b) Cross-sectional sentinel surveillance syndrome aetiologies and HPV genotypes between 2014-2015 in 36 healthcare facilities across South Africa and presently on GERMS-SA platform with incorporates STI aetiologies, antimicrobial resistance and HPV genotyping. We are reporting the impact this surveillance had on the control and management of STIs in South Africa, the progress that has been made, the new approach and challenges that this programme is facing and suggestions how it can be improved.

\section{Methods}

Sentinel sites for surveillance was undertaken yearly or biennially across the country in all nine provinces. Selection was based on the total volume of STI patient seen yearly in a particular clinic and presently on number of MUS cases ( $\geq 25$ per month). All patients 18 years and above complaining of one of the three syndromes were recruited with informed consent. Genital and blood samples were collected in addition to screening of 100 asymptomatic women aged 18-20 for HPV genotyping

\section{Results}

The positive impact on STI control and management illustrated by the change and introduction of new STIs management guidelines in 2008 and recently 2015 in support of STI syndromic management. The information gathered prompted the NDOH to rethink about a new approach to syphilis and the initiation of a new comprehensive STI strategy for South Africa.

\section{Conclusion}

The impact, challenges, shortcomings and variables identified that hinder progress on STI surveillance will be discussed in detail in this presentation, as well as the detailed new approach incorporated into GERMS-SA.

\section{ID: 8327}

Category: Clinical Microbiology (SASCM)

Permission: Yes

Author: Centner, $C$ 
Author Institutes: National Health Laboratory Service, University of Cape Town

\section{E-mail Address: chadc@nis.za}

Country: South Africa

\section{ABSTRACT TITLE: TRAK HELPER: SOFTWARE TO AUTOMATE MANUAL ENTRY OF MICROBIOLOGY TEST RESULTS ON THE LABORATORY INFORMATION SYSTEM}

\section{Introduction}

Despite increasing laboratory automation, many routine clinical microbiology test results are generated manually. Manual entry of results in the laboratory information system (LIS) can be laborious and time-consuming, involving many user interface steps for a single result. At the National Health Laboratory Service microbiology laboratory at Groote Schuur Hospital, we developed and implemented Trak Helper software to "overlay" TrakCare Lab LIS (InterSystems, Cambridge, MA). Trak Helper is written in AutoHotkey, a free open-source macro scripting language for Microsoft Windows. Trak Helper consists of $>30$ applications and shortcuts that automate manual LIS tasks by emulating user interface steps (mouse clicks, keyboard entries). We evaluated the benefit of Trak Helper in reducing manual steps and hands-on time on a subset of test results.

\section{Methods}

At our laboratory, approximately 80 negative (no growth) CSF culture results are entered manually per day. We compared the number of steps and time taken to enter a negative CSF culture result manually versus using Trak Helper. Two technologists were timed entering all negative CSF culture results over six to 11-day periods per method.

\section{Results}

Trak Helper reduced the number of steps required to enter a negative CSF culture result from twelve or six steps (depending on the age of the sample) to a single step. For Technologist 1, time per sample was reduced from a median of 39 seconds (IQR 38-41) to 23 seconds ( $21-29)$ ( $41 \%$ improvement, $p=0.002$ ). Improvement for technologist 2 was $33 \%(p=0.028)$. Median hands-on time per day was reduced from 54 minutes to 31 minutes for Technologist 1, and from 45 minutes to 30 minutes for Technologist 2 .

\section{Conclusion}

A simple software application reduced hands-on time for negative CSF culture result entry on the LIS by up to $41 \%$, saving the laboratory up to 15 person-days/year of skilled technologist time. Additional savings could be gained by further software development and wider implementation.

\section{ID: 8383}

Category: Clinical Microbiology (SASCM)

Permission: Yes

Co-Author 1: van Schalkwyk, E
Co-Author 1 Institute: Centre for Healthcare-Associated Infections, Antimicrobial Resistance and Mycoses, National Institute for Communicable Diseases

Co-Author 2: Quan, V

Co-Author 2 Institute: GERMS-SA programme, Division of Public Health Surveillance and Response, National Institute for Communicable Diseases

Co-Author 3: Govender, N

Co-Author 3 Institute: Centre for Healthcare-Associated Infections, Antimicrobial Resistance and Mycoses, National Institute for Communicable Diseases, Faculty of Health Sciences, University of the Witwatersrand

\section{Co-Author 4: Perovic, 0}

Co-Author 4 Institute: Centre for Healthcare-Associated Infections, Antimicrobial Resistance and Mycoses, National Institute for Communicable Diseases, Faculty of Health Sciences, University of the Witwatersrand

E-mail Address: erikab@nicd.ac.za

Country: South Africa

ABSTRACT TITLE: CLINICAL CHARACTERISTICS ASSOCIATED WITH OUTCOME IN PATIENTS WITH COMMUNITY-VERSUS HOSPITAL-ASSOCIATED METHICILLIN-RESISTANT STAPHYLOCOCCUS AUREUS BLOODSTREAM INFECTION IN FIVE SOUTH AFRICAN HOSPITALS

\section{Introduction}

Methicillin-resistant Staphylococcus aureus (MRSA) is a major cause of healthcare-associated infections. We compared clinical characteristics and outcome of patients with community-associated (CA) MRSA and healthcare-associated (HA) MRSA infections.

\section{Methods}

We analysed active, laboratory-based surveillance data for $S$. aureus bloodstream infection (BSI), 2013 through 2015, at five sentinel hospitals. A case of MRSA BSI was defined as any patient with S. aureus, non-susceptible to oxacillin or cefoxitin, cultured from blood within a 21-day period. CA-MRSA was defined as MRSA isolated within 48 hours of hospital admission and no admission within the previous year. A reference laboratory confirmed isolate identification and antimicrobial susceptibility. We used multivariable logistic regression to evaluate factors associated with CA-MRSA versus HA-MRSA, and compare in-hospital outcome between the two groups.

\section{Results}

Among 557 patients with MRSA BSI, 44 cases (7.9\%) had CAMRSA and 513 (92.1\%) had HA-MRSA; 60\% were male (335/555). The median age was three years (IQR: 0-42), with no difference between the two groups ( $p=0.816)$. Thirty-nine per cent of CAMRSA (17/44) and 47\% of HA-MRSA cases (239/512) were aged $<1$ year. HIV-infected patients were three times more likely to have CA-MRSA than HA-MRSA, after adjustment for age and sex (aOR:3.3; $95 \% \mathrm{Cl}: 1.1-9.3$ ). Fever (body temperature $\geq 37.5^{\circ} \mathrm{C}$ ) at diagnosis was associated with HA-MRSA (aOR: $0.2 ; 95 \% \mathrm{Cl}: 0.05-0.7$ ). Crude in-hospital mortality was $41.2 \%$. There was no association between 
MRSA category and outcome. Increasing age (age 50-59 aOR: 3.14; 95\% Cl: $1.61-6.13$, age $\geq 60$ aOR: $5.15 ; 95 \% \mathrm{Cl}: 2.50-10.60$, compared to age < 1 year), cardiac arrest (aOR: 23.03; 95\% Cl: 2.88-183.93), mechanical ventilation (aOR: 1.89; $95 \% \mathrm{Cl}: 1.21-2.95)$ and prior MRSA infection (aOR: $3.44 ; 95 \% \mathrm{Cl}: 1.38-8.53$ ) were associated with mortality among all cases.

\section{Conclusion}

HIV infection and fever could predict CA-MRSA and HA-MRSA, respectively. There was no outcome difference between CA-MRSA and HA-MRSA cases.

\section{ID: 8384}

Category: Clinical Microbiology (SASCM)

Permission: Yes

Co-Author 1: Mvelase, NR

Co-Author 1 Institute: Department of Medical Microbiology, KwaZulu-Natal Academic Complex, National Health Laboratory Service; Department of Medical Microbiology, School of Laboratory Medicine \& Medical Sciences, University of KwaZulu-Natal

Co-Author 2: Pillay, M

Co-Author 2 Institute: Department of Medical Microbiology, KwaZulu-Natal Academic Complex, National Health Laboratory Service

Co-Author 3: Mlisana, KP

Co-Author 3 Institute: Department of Medical Microbiology, KwaZulu-Natal Academic Complex, National Health Laboratory Service; Department of Medical Microbiology, School of Laboratory Medicine \& Medical Sciences, University of KwaZulu-Natal

E-mail Address: dlaminin15@ukzn.ac.za

Country: South Africa

\section{ABSTRACT TITLE: RPOB MUTATIONS CAUSING DISCORDANT RIFAMPICIN SUSCEPTIBILITY IN \\ MYCOBACTERIUM TUBERCULOSIS IN KWAZULU-NATAL, SOUTH AFRICA}

\section{Background}

The rapid diagnosis of drug resistant tuberculosis is limited by the discrepancy in drug susceptibility results between molecular and genotypic assays. Our aim was to determine the prevalence of phenotypically susceptible rpoB mutations as well as to characterise them so as to define optimal ways of diagnosis.

\section{Methods}

Discordant clinical isolates showing rifampicin resistance on GenoType MTBDRplus while susceptible on 1\% agar proportion method using Middlebrook $7 \mathrm{H} 10$ agar were collected from KwaZuluNatal provincial TB laboratory between May and December 2014. These were tested using agar dilution (AD) in Middlebrook 7H10 and automated Bactec Mycobacteria Growth Indicator Tube (MGIT) 960 MICs. Additionally, subsets of stored discordant as well as 40 fully susceptible isolates were included. Sequencing was performed on all discordant isolates.

\section{Results}

Discordant isolates constituted 5\% (67) of 1324 rifampicin resistant isolates detected by MTBDRplus during the study period. Out of 90 discordant isolates (including 23 stored isolates); sequencing confirmed the presence of rpoB mutations in all isolates. The most frequent mutation was Gln513Pro at 24 (26\%), followed by 17 (19\%) Asp516Val and 13 (14\%) Asp516Tyr. The majority of MICs were between 0.5 and $2 \mu \mathrm{g} / \mathrm{ml}$. ( $84 \%$ for $A D$ and $74 \%$ for MGIT). Isoniazid resistance was found in $34(37 \%)$ isolates and it was associated with higher rifampicin MICs on the AD. Among the 40 fully susceptible isolates (sensitive to isoniazid and rifampicin -wild type rpoB gene) $38(95 \%)$ had a rifampicin MIC of $\leq 0.25 \mu \mathrm{g} / \mathrm{ml}$.

\section{Conclusions}

Given the high burden of disease in our setting, the isolates with discordant genotypic and phenotypic susceptibility results are not uncommon. Their association with MICs around critical concentration plus isoniazid resistance raises concerns regarding clinical implications as these may represent pre-MDR TB phenomenon. The frequent occurrence of an uncommon mutation, 513Pro warrants further investigation in order to define its public health implications.

\section{ID: 8292}

Category: Infection Control (ICSSA)

Permission: No

Co-Author 1: Dowling, W

Co-Author 1 Institute: National Health Laboratory Service

Co-Author 2: Singh, $S$

Co-Author 2 Institute: National Health Laboratory Service

E-mail Address: wbdowling@gmail.com

Country: South Africa

\section{ABSTRACT TITLE: INVESTIGATION OF A POSSIBLE RALSTONIA INSIDIOSA OUTBREAK IN A REGIONAL HOSPITAL IN THE WESTERN CAPE}

\section{Introduction}

Ralstonia insidiosa is a Gram-negative bacillus that has rarely been associated with infections in humans. We report a pseudo-outbreak of this organism in a regional hospital in the Western Cape.

\section{Methods}

We performed an outbreak investigation following a clustering of 18 isolates on blood cultures received over a two-month period. The investigation included laboratory diagnosis and environmental sampling. Blood culture isolates were identified by Vitek $^{\circledast} 2$ automated identification system and confirmation performed on two isolates by mass spectrometry. Environmental samples were confirmed on Vitek ${ }^{\circledR} 2$ and mass spectrometry. 


\section{Results}

Eighteen blood culture isolates were identified as Cupriavidus pauculus by the Vitek 2 platform. All index blood cultures were taken from casualty or paediatric wards and had similar antibiograms. No patients had symptoms suggestive of C.pauculus infection and there was no epidemiological link between patients The mass spectrometry platform identified the two patient samples as R.insidiosa. We subsequently identified R.insidiosa during initial environmental sampling from diluted chlorhexidine skin cleaning solution. We confirmed the source to be the chlorhexidine solution from a storage container in pharmacy. A private laboratory confirmed R.insidiosa from this sample. No relevant organisms were found in any other solutions, blood culture bottles or tap water.

\section{Conclusion}

Incorrect diluting and storage practices of chlorhexidine lead to the pseudo-outbreak of R.insidiosa. Ralstonia spp and C.pauculus are two closely related Gram-negative bacteria that are almost indistinguishable with automated lab identification systems. Identification of these organisms in the microbiology laboratory should alert clinicians to a potential pseudo-outbreak.

\section{ID: 8438}

Category: Infection Control (ICSSA)

Permission: Yes

Author: Grau-Pujol, B

Author Institute: Centro de Investigação em Saúde da Manhiça

E-mail Address: berta.grau@isglobal.org

Country: Mozambique

\section{ABSTRACT TITLE: WATER SUPPLY AND SANITATION CONDITIONS IN RURAL SOUTHERN MOZAMBIQUE AND ITS ASSOCIATION WITH MORBIDITY INDICATORS DURING 2012-2015}

\section{Introduction}

Water and sanitation (WASH) are major health determinants, responsible for $5.7 \%$ of worldwide disease burden. However, the debate about the effect of water quality and sanitation in morbidity such as diarrhoea is still ongoing. The aim of this study is to describe access to improved WASH infrastructure at a community and household level, as defined by the Joint Monitoring Programme, during 2012-2015 in the Manhiça Health Research Centre (CISM) study area and evaluate its association with morbidity indicators

\section{Methods}

We conducted a retrospective cohort study. We included all children under 15 living in the study area during the period 2012-2015 $(\mathrm{N}=61,900)$. Children were followed up until they moved from the study area, turned 15 or until 2015. WASH household data was obtained from the CISM demographic surveillance system in the Manhiça district. Clinical data was obtained from CISM roundthe-clock morbidity surveillance system covering outpatient and hospital admissions at the Manhiça District Hospital and rural health posts. A negative binomial regression model using Wald test was performed to assess the minimum community-based incidence rates for every morbi-mortality indicator. WASH community herd protection for morbidity indicators was assessed spatially using QGIS.

\section{Results}

Water access conditions enhanced in 2015(85\% children living in improved conditions) compared to 2012 (73\%). However, $75 \%$ of children remained in unimproved sanitation conditions during this period. Spatial distribution of unimproved water and sanitation facilities showed to be clustered. Access to unimproved sanitation and water facilities were associated to higher rates of malaria parasitemia, which adjusted incidence rate increase was doubled. Other studied morbidity indicators such as diarrhoea, malnutrition and dehydration did show weaker evidence of association between the use of unimproved water and sanitation facilities and its adjusted incidence rate. We did not observed community herd protection by owing improved water and sanitation facilities.

\section{Conclusion}

Achieved results are useful to inform sector-related decisionmaking processes and ultimately improve access to safe drinking water and sanitation in rural southern Mozambique.

\section{ID: 8291}

Category: Infection Control (ICSSA)

Permission: Yes

Co-Author 1: Nel, P

Co-Author 1 Institute: Stellenbosch University

Co-Author 2: Hoffmann, R

Co-Author 2 Institute: Stellenbosch University

E-mail Address: panquarius@gmail.com

Country: South Africa

\section{ABSTRACT TITLE: A POINT-PREVALENCE STUDY OF ADULT IN-PATIENTS TO DETERMINE COLONISATION WITH CARBAPENEMASE-PRODUCING ENTEROBACTERIACEAE IN TYGERBERG HOSPITAL, WESTERN CAPE, SOUTH AFRICA}

\section{Introduction}

The prevalence of carbapenemase-producing Enterobacteriaceae (CPE) has been increasing around the world in recent years, but data regarding the prevalence and clinical significance of CPE in Africa is not well documented. To determine the current prevalence of CPE colonisation a point-prevalence study was performed at TBH's adult in-patient wards. The need for routine screening and risk factors associated with colonisation were also investigated.

\section{Methods}

A total of 439 adult in-patients from 46 different wards in TBH were screened with rectal swabs during a three-month period. Risk factors 
were recorded for each patient. Patient samples were tested for carbapenem-resistant Enterobacteriaceae using a MacConkey agar plate with an ertapenem disc and the Vitek ${ }^{\circledR} 2$ system. Preliminary positive samples were tested for carbapenemase-production with a multiplex PCR containing primers for five associated genes.

\section{Results}

Six hundred adult in-patients were approached for participation in this study and 161 (25.33\%) withheld consent. Of the 439 patient samples collected, $12(2.7 \%)$ screened positive for carbapenemresistance and of these, one $(0.22 \%)$ sample was positive for carbapenemase-production. This patient was colonised with a Klebsiella pneumoniae organism harbouring a NDM-1-type gene and had no commonly reported risk factors associated with CPE colonisation.

\section{Conclusion}

It seems that the routine screening of adult patients for CPEcolonisation during admission to TBH is unwarranted. Risk factors associated with CPE-colonisation in this specific setting require further clarification.

\section{ID: 8494}

Category: Infectious Diseases (IDSSA)

Permission: No

Co-Author 1: Oberholster, L

Co-Author 1 Institute: Department of Microbial, Biochemical and Food Biotechnology, University of the Free State

Co-Author 2: Aschenbrenner, J

Co-Author 2 Institute: School of Life Sciences, Technical University of Munich

Co-Author 3: Potgieter, $C$

Co-Author 3 Institute: Deltamune (Pty.) Ltd.

Co-Author 4: O'Neill, HG

Co-Author 4 Institute: Department of Microbial, Biochemical and Food Biotechnology, University of the Free State

E-mail Address: lariseoberholster@gmail.com

Country: South Africa \& Germany

\section{ABSTRACT TITLE: ENGINEERING NEWCASTLE DISEASE VIRUS AS VACCINE DELIVERY SYSTEM FOR ROTAVIRUS VP7 AND NSP4}

\section{Introduction}

Rotavirus (RV) is the leading cause of severe dehydrating diarrhoea in young children. Due to the zoonotic nature of RV infections, the young of various animals, including livestock, are also affected, resulting in devastating economic losses. Recent reports have described animal-human reassortant strains, such as bovine-human reassortants. These interspecies transmitted strains contribute to the diversity of rotavirus strains circulating in humans and emphasise the need for a One Health approach in RV control. Infection occurs in calves 2-4 weeks of age indicating the protective role of maternal antibodies in the colostrum immediately postpartum. Vaccination remains the only existing prophylactic measure against infection and aims to increase the level of anti-RV antibodies in the colostrum. Newcastle disease virus is an attractive vaccine delivery vector for mammals because of its natural host-range restriction in non-avian species. It is also antigenically distinct from common animal and human pathogens and would, therefore, not be affected by a preexisting immunity against NDV.

\section{Methods}

Recombinant NDVs were constructed to express the outer-capsid glycoprotein (VP7) and the enterotoxin non-structural protein (NSP4) of RV. The recombinant viruses were rescued in BSR-T7 cells and passaged in SPF embryonated chicken eggs. RNA was extracted from allantoic fluid and sequenced using the Illumina MiSeq platform for verification of successful gene insertion. Protein expression was verified using immunoperoxidase monolayer assay (IPMA) and western blot analysis.

\section{Results}

Recombinant NDVs containing open reading frames encoding rotavirus VP7 and NSP4 were constructed and successfully rescued from BSR-T7 cells. The viability of recombinant NDVs were confirmed using the hemagglutination assay, virus titrations and sequencing. Expression of rotavirus proteins was subsequently confirmed from BSR-T7 and MDBK cells.

\section{Conclusion}

This is the first step towards a possible vaccine vector for RV infection in bovine for a One Health approach to RV control.

\section{ID: 8459}

Category: Infectious Diseases (IDSSA)

Permission: Yes

Co-Author 1: Smith, A

Co-Author 1 Institute: National Institute for Communicable Diseases (NICD)

Co-Author 2: Tau, N

Co-Author 2 Institute: National Institute for Communicable Diseases (NICD)

Co-Author 3: Bosco, K

Co-Author 3 Institute: University of Cape Town

Co-Author 4: Ismail, A

Co-Author 4 Institute: National Institute for Communicable Diseases (NICD)

Co-Author 5: Allam, M

Co-Author 5 Institute: National Institute for Communicable Diseases (NICD)

Co-Author 6: Keddy, K

Co-Author 6 Institute: National Institute for Communicable Diseases (NICD) 
E-mail Address: anthonys@nicd.ac.za

Country: South Africa

\section{ABSTRACT TITLE: WHOLE-GENOME SEQUENCING AND MICROBIOME ANALYSIS TO IDENTIFY AND CHARACTERISE SHIGA TOXIN-PRODUCING ESCHERICHIA COLI FROM THE STOOL OF A PATIENT PRESENTING WITH HAEMOLYTIC URAEMIC SYNDROME}

\section{Introduction}

Microbial whole-genome sequencing (WGS) holds great promise for enhancing diagnostic and public health microbiology. WGS analysis of bacterial cultures is well established, but when applied directly on clinical samples is more challenging. We describe the use of WGS and microbiome analysis applied directly on a culturenegative stool sample, from a patient presenting with haemolytic uraemic syndrome (HUS), to successfully identify and characterise Shiga toxin-producing Escherichia coli (STEC).

\section{Methods}

Total DNA was extracted from stool samples using the MagNA Pure LC Total Nucleic Acid Isolation Kit. DNA was further processed using the NEBNext Microbiome DNA enrichment Kit. Enriched microbial DNA samples were subjected to WGS using Illumina MiSeq next generation sequencing technology. Raw sequencing data were analysed against microbial databases using SURPI (sequencebased ultra-rapid pathogen identification), a computational bioinformatics pipeline for pathogen identification utilising complex metagenomic sequencing data. Raw sequencing data were further analyzed using CLC Genomics Workbench Software; trimmed reads were assembled de novo. Assembled data were analysed using bioinformatics pipelines available at the Center for Genomic Epidemiology (CGE) (http://cge.cbs.dtu.dk/services/).

\section{Results}

SURPI analysis against bacterial databases returned an overwhelming and convincing match to E. coli; additionally, SURPI analysis against viral databases found matches to phages which house stx2 genes (markers for STEC). Analysis of assembled data at the CGE identified STEC with the following characteristics: serotype 026:H11; presence of eae, st $2 \mathrm{a}$ and $\mathrm{st} \times 2 \mathrm{~b}$ virulence genes; multilocus sequence typing (MLST) sequence type 21 .

\section{Conclusion}

WGS and microbiome analysis was able to convincingly identify and characterise STEC from a culture-negative stool sample. If implemented in real-time, WGS applied directly on clinical specimens can drastically reduce diagnostic times and rapidly provide clinically relevant and actionable public health information. This has significant patient management and huge public health benefits, especially for enhancing turn-around times for the diagnosis and characterisation of outbreak-prone pathogens including STEC.

\section{ID: 8280}

Category: Infectious Diseases (IDSSA)

Permission: No

Co-Author 1: Van Schalkwyk, M

Co-Author 1 Institute: Stellenbosch University

Co-Author 2: Bekker, A

Co-Author 2 Institute: Stellenbosch University

Co-Author 3: Decloedt, E

Co-Author 3 Institute: Stellenbosch University

E-mail Address: marije@sun.ac.za

Country: South Africa

ABSTRACT TITLE: PHARMACOKINETICS OF RIFAMPIN AND ISONIAZID DURING PREGNANCY AND POSTPARTUM IN SOUTH AFRICAN WOMEN

\section{Introduction}

Physiological changes during pregnancy may alter drug pharmacokinetics. Trimester differences in rifampin (RMP) and isoniazid (INH) exposure have not been described. We explored the effects of pregnancy gestation on RMP and INH pharmacokinetics in tuberculosis-infected women.

\section{Methods}

P1026s is a prospective study of antiretroviral and anti-tuberculosis pharmacokinetics in HIV-infected and uninfected pregnant women. Intensive 24-hour pharmacokinetic profiles of RMP and INH were performed during the 2 nd trimester $(2 \mathrm{~T}), 3 \mathrm{rd}$ trimester $(3 \mathrm{~T})$, and 2-8 weeks postpartum(PP). Daily anti-tuberculosis tablets were given according to WHO-recommended guidelines. RMP and INH plasma concentrations were measured using High Performance Liquid Chromatography(HPLC); detection limits being $0.117 \mu \mathrm{g} / \mathrm{ml}$ and $0.098 \mu \mathrm{g} / \mathrm{ml}$ respectively. The pharmacokinetic parameters were characterised using noncompartmental analysis and compared to published non-pregnant South African adult data.

\section{Results}

Preliminary pharmacokinetic data are available for ten South African participants; seven African, two mixed descent, and one Indian. Eight women were HIV-infected (seven on efavirenz and one on lopinavir/ritonavir). Median age at 3T was 31 years (range 21-40) and median weight at 3T was $58.6 \mathrm{~kg}$ (range 49-99). Median gestational age at delivery was 38 weeks (range 36-41). RMP and INH pharmacokinetic data were available in 5,8 and three women in $2 \mathrm{~T}, 3 \mathrm{~T}$, and PP. Compared to a non-pregnant South African adult cohort (45\% male, 10\% HIV-infected not receiving antiretrovirals, Mcllleron et al. 2006), RMP exposure was similar or higher in $2 \mathrm{~T}$ and 3T. INH exposure was below the 25th percentile across all stages of pregnancy. Small sample size and unavailable comparator raw dataset prohibited formal statistical testing.

\section{Conclusion}

RMP concentrations in pregnancy compared well to non-pregnant concentrations. INH exposure was reduced throughout pregnancy. 
If confirmed with larger sample size, an increased dose of INH may be needed during pregnancy.

\section{ID: 8356}

Category: Clinical Microbiology (SASCM)

Permission: Yes

Co-Author 1: Bhola, P

Co-Author 1 Institute: Department of Medical Microbiology, National Health Laboratory Services, School of Laboratory Medicine and Medical Sciences, University of KwaZulu Natal

Co-Author 2: Mvelase, NR

Co-Author 2 Institute: Department of Medical Microbiology, National Health Laboratory Services, School of Laboratory Medicine and Medical Sciences, University of KwaZulu Natal

Co-Author 3: Mlisana, KP

\section{Co-Author 3 Institute:}

E-mail Address: bholap@ukzn.ac.za

Country: South Africa Department of Medical Microbiology, National Health Laboratory Services, School of Laboratory Medicine and Medical Sciences, University of KwaZulu Natal

\section{ABSTRACT TITLE: URINARY TRACT INFECTIONS IN PREGNANT WOMEN IN KZN: BACTERIAL AETIOLOGY AND SUSCEPTIBILITY}

\section{Introduction}

Urinary tract infection (UTI) is one of the commonest infections during pregnancy. Untreated, it can cause significant maternal and perinatal morbidity and mortality. Challenges while treating UTIs in pregnancy include foetal protection and resistance development of uropathogens. Currently, the Essential Drug List (EDL) recommends amoxicillin-clavulanic-acid to treat cystitis and ceftriaxone/ gentamicin for pyelonephritis. Nitrofurantoin is not included in the recommendation. Cefuroxime has been removed from the EDL.Aim: To determine common pathogens causing UTI in pregnancy and their antibiotic susceptibility patterns.

\section{Methods}

A retrospective analysis of laboratory data for positive urine specimens from obstetric departments of six KZN hospitals during 2012-2016 was performed. Identification and susceptibility testing was performed using the Vitek2 system. Antibiotics tested were amoxicillin, cephalothin, cefuroxime, ceftriaxone, nitrofurantoin, amoxicillin-clavulanic-acid, ciprofloxacin, gentamicin, and cotrimoxazole. Results were interpreted according to Clinical and Laboratory Standards Institute (CLSI) guidelines.

\section{Results}

From 5855 urine specimens, the commonest isolate was E coli, 2984 (51.2\%), K pneumoniae, 718 (12.2\%), C albicans, 377 (6.4\%), Candida species, 292 (3.2\%), Group B Streptococcus(GBS), 225 (3.8\%), E faecalis, 218 (3.7\%), and $17.7 \%$ various others. E coli displayed $71.9 \%$ resistance to ampicillin, cotrimoxazole (65.2\%), cephalothin (23.7\%) and ciprofloxacin (12.3\%). Resistance remained low to cefuroxime $4.1 \%$, ceftriaxone $1.8 \%$, amoxicillin-clavulanicacid $5.3 \%$, gentamicin $5.5 \%$ and nitrofurantoin $2.8 \%$. K pneumoniae was similar. GBS displayed $99.5 \%$ penicillin susceptibility. E faecalis displayed $92 \%$ susceptibility to ampicillin, amoxicillin-clavulanicacid, and nitrofurantoin.

\section{Conclusion}

E coli is unsurprisingly the most common cause of UTI in pregnancy (KZN), with > 94\% susceptibility to cefuroxime, ceftriaxone, amoxicillin-clavulanic-acid, nitrofurantoin and gentamicin. GBS remains prevalent, and susceptible to penicillin. E faecalis remains susceptible to ampicillin and nitrofurantoin. Candida featured prominently in this analysis, and its significance needs further studying. To spare ceftriaxone, nitrofurantoin could be an alternative in treating cystitis and cefuroxime for pyelonephritis. As drug resistance is evolving, routine surveillance is necessary to provide updated information on recommended antibiotic use.

\section{ID: 8453}

Category: Clinical Microbiology (SASCM)

Permission: Yes

Co-Author 1: Bolukaoto, YJ

Co-Author 1 Institute: University of Pretoria

Co-Author 2: Ehlers, MM

Co-Author 2 Institute: University of Pretoria, National Health Laboratory Service

Co-Author 3: Kock, MM

Co-Author 3 Institute: University of Pretoria, National Health Laboratory Service

Co-Author 4: Strydom, K

Co-Author 4 Institute: University of Pretoria, National Health Laboratory Service

E-mail Address: jbolukaoto@gmail.com

Country: South Africa

\section{ABSTRACT TITLE: VIRULENCE PROFILES OF ENTEROHAEMORRHAGIC ESCHERICHIA COLI 0157:H7 ISOLATES FROM CLINICAL SPECIMENS AND ENVIRONMENTAL SAMPLES IN GAUTENG REGION, SOUTH AFRICA}

\section{Introduction}

Enterohaemorrhagic Escherichia coli (EHEC) 0157:H7 or Shigatoxins producing $\mathrm{E}$. coli is an important gastrointestinal pathogen causing bloody diarrhoea and more severe diseases such as haemolytic uremic syndrome, an important cause of acute kidney failure in children. The reservoirs for EHEC 0157:H7 are healthy ruminant animals. Humans acquire EHEC 0157:H7 by direct contact with animal or via the ingestion of contaminated food or water. In South Africa, limited information is available on characterisation of EHEC 0157:H7. The aim of this study was to determine the virulence 
profile of EHEC 0157:H7 isolates from clinical specimens and environmental water samples in the Gauteng region of South Africa.

\section{Methods}

Clinical stool specimens and environmental water samples were collected from October 2016 to June 2017. Samples were cultured on selective chromogenic media and presumptive EHEC 0157:H7 were confirmed by using a latex agglutination test. Isolates were characterised using multiplex PCR assays to screen for the uidA rfbEO157 and fliCH7 genes that encode for $\beta$-glucuronidase, somatic- $\mathrm{O}$ and flagellar-H antigens, respectively and for the virulence genes: Shiga-toxins 1 and 2 (Stx-1 \& Stx-2), enterohaemolysin (hlyA) and $E$. coli attaching and effacing lesion (eae).

\section{Results}

A total of 213 samples were analysed of which $10.7 \%$ (23/213) were identified as EHEC 0157:H7 by M-PCR assays. Run-off water represented $86.9 \%(20 / 23)$ of isolates and stool specimens represented $13 \%(3 / 23)$. The Stx-1, Stx-2, hlyA and eae genes were present in $7.6 \%(2 / 23), 15.3 \%(4 / 23), 3.8 \%(1 / 23)$ and $11.55 \%(3 / 23)$ of isolates, respectively. Virulence genes were not detected in $47.8 \%$ $(11 / 23)$ of isolates.

\section{Conclusion}

This study reported the presence of EHEC 0157:H7 in stool specimens and environmental water samples, showing the under reporting and miss diagnosis of $13 \%$ of stool specimens due to the diagnostic screening test used. Environmental surveillance plays an important role in reducing the potential health risk to consumer of untreated water. The inclusion of molecular testing for EHEC 0157:H7 of stool specimen can improve the detection of this important pathogen in this clinical setting.

\section{ID: 8673}

Category: Clinical Microbiology (SASCM)

Permission: Yes

Co-Author 1: Dreyer, A

Co-Author 1 Institute: Vermaak and Partners Pathologists, Pathcare

Co-Author 2: Marcus, L

Co-Author 2 Institute: Vermaak and Partners Pathologists, Pathcare

Co-Author 3: Ahmed, K

Co-Author 3 Institute: Vermaak and Partners Pathologists, Pathcare Co-Author 4: Nyapholi, M

Co-Author 4 Institute:Vermaak and Partners Pathologists, Pathcare Co-Author 5: Hoosen, A

Co-Author 5 Institute:Vermaak and Partners Pathologists, Pathcare Co-Author 6: Lowman, W

Co-Author 6 Institute:Vermaak and Partners Pathologists, Pathcare E-mail Address: aw.dreyer@gmail.com

Country: South Africa

\section{ABSTRACT TITLE: RECONSIDERING CLOSTRIDIUM DIFFICILE TESTING IN SA - A CRITICAL LOOK AT THE NEED FOR A TWO-STEP ALGORITHM!}

\section{Introduction}

Current Clostridium difficile diagnostic assays are inadequate as stand-alone tests. Molecular assays show greater sensitivity compared to enzyme immune assays (EIAs) in detecting toxigenic strains however, lack the ability to discriminate between active disease (toxin production) and colonisation.

\section{Methods}

A retrospective analysis was performed using specimens submitted for C. difficile testing during the period 5 May 2016 to 30 April 2017 from the private health sector in Gauteng. Results from samples tested with both Xpert C. difficile (Cepheid) and the C Diff Quick Check Complete (Alere) were included. Sensitivity, specificity, positive predictive value (PPV) and negative predictive value (NPV) of the glutamate dehydrogenase (GDH) antigen was determined, using PCR as the reference. Toxin detection (TcdA/B) was expressed as a percentage of PCR positives. Association between sample consistency (solid, semisolid and liquid) was determined.

\section{Results}

A total of 165 samples were included for analysis of which 113 (68.5\%) were negative by PCR testing. Toxin was detected in 14 (30\%) of the PCR positives. Diagnostic performance of the GDH antigen demonstrated: sensitivity (84\%), specificity (91\%), PPV (78\%) and NPV (93\%). The majority (53\%) of the samples were semiformed and no association between the consistency and toxin production or carriage could be determined.

\section{Conclusion}

Toxin positivity correlates best with clinical outcome and assists in targeting patients for treatment. Up to $70 \%$ of patients may have received unnecessary antimicrobial therapy. The sensitivity of the GDH antigen to detect and inability to exclude disease limits its use as a screening test compared to PCR, seven (4\%) of patients would have been missed in the study group with EIA-based testing alone. Our findings support an algorithmic approach to diagnosis of C. difficile associated diarrhea. We recommend a PCR based assay with subsequent toxin detection of positives to distinguish between active disease and colonisation.

\section{ID: 8497}

Category: Clinical Microbiology (SASCM)

Permission: Yes

Co-Author 1: du Plooy, I

Co-Author 1 Institute: Ampath

Co-Author 2: Coetzee, J

Co-Author 2 Institute: Ampath

Co-Author 3: Prentice, E

Co-Author 3 Institute: Ampath 
Co-Author 4: Clay, C

E-mail Address: dp.ilze@gmail.com

Country: South Africa

\section{ABSTRACT TITLE: COMPARISON OF DIAGNOSTIC TESTS FOR CLOSTRIDIUM DIFFICILE IN A PRIVATE SECTOR LABORATORY IN GAUTENG, SOUTH AFRICA}

\section{Introduction}

The diagnosis of Clostridium difficile infection is a frequently debated topic due to the wide range of diagnostic strategies. The objective of this study was to compare diagnostic tests for $C$. difficile performed in a private laboratory in Gauteng.

\section{Methods}

A convenience sample of 100 consecutive liquid PCR positive stool specimens (BD MAX ${ }^{\mathrm{TM}}$ Cdiff) were selected and further tested for GDH and toxin A and B (CerTest Clostridium difficile, BIOTEC S.L.). All were also cultured using selective agar (chromiD ${ }^{\circledR} \mathrm{C}$. difficile agar, Biomerieux). Culture positive isolates were confirmed to be toxigenic by use of PCR (Roche LightMix ${ }^{\circledast}$ Kit Clostridium difficile, TibMolBiol.) which also detected the 18-bp sequence deletions in the tcdC gene associated with hypervirulence.

\section{Results}

Of the 100 PCR positive stools, 95 were culture positive and toxigenic. Of these 90 were GDH positive. 36 were toxin positive of which the majority, $72 \%(26 / 36)$, were positive for Toxin A and B. 2 strains showed the 18-bp deletion in the $\mathrm{tcd} C$ gene, indicative of a hyper-virulent strain.

\section{Conclusion}

Most PCR positive stools selected were culture positive for toxigenic strains. The detection of toxin in only 36 of $95 \mathrm{C}$. difficile culture positive stools is a higher rate of discrepant results than reported in most studies. The absence of $C$. difficile toxins in EIA tests should not rule out the possibility of $\mathrm{CDI}$. As toxin detection is dependent on the level of toxin production at sample collection and the sensitivity of the test, this magnitude of discrepancy may also be due in part to the kit used in this study. The use of PCR remains important.

\section{ID: 8408}

Category: Clinical Microbiology (SASCM)

Permission: Yes

Co-Author 1: Geldenhuys, J

Co-Author 1 Institute: Department of Medical Microbiology, Faculty of Health Sciences, University of Pretoria

Co-Author 2: Valverde, A

Co-Author 2 Institute: Centre for Microbial Ecology and Genomics (CMEG), Genomics Research Institute, Department of Genetics, University of Pretoria

Co-Author 3: Lombaard, HA

Co-Author 3 Institute: Obstetrics and Gynecology, Rahima Moosa
Mother and Child Hospital, Wits Obstetrics and Gynecology Clinical Research Division, School of Clinical Medicine, Faculty of Health Science

Co-Author 4: Ehlers, MM

Co-Author 4 Institute: Department of Medical Microbiology, Faculty of Health Sciences, University of Pretoria

Co-Author 5: Kock, MM

Co-Author 5 Institute: Department of Medical Microbiology, Faculty of Health Sciences, University of Pretoria

E-mail Address: geldenhuysjanri@gmail.com

Country: South Africa

\section{ABSTRACT TITLE: DIVERSITY OF THE GUT, VAGINAL AND ORAL MICROBIOME AMONG PREGNANT WOMEN IN SOUTH AFRICA WITH PRE-ECLAMPSIA}

\section{Introduction}

Pre-eclampsia is a multisystem disorder better known as a condition of high blood pressure and proteinuria associated with pregnancy. It is considered a leading cause of maternal as well as fetal/neonatal morbidity and mortality. Pre-eclampsia has a complex aetiology of which the exact cause remains unknown; however, its association with exaggerated systemic inflammation suggests microbial infection and a bacterial source to play a possible role.

\section{Methods}

Oral, vaginal and rectal samples were collected from ten primiparous pregnant women in both the normotensive and preeclampsia group. Targeted 16S rRNA next generation sequencing (NGS) was performed using an Illumina MiSeq platform. Analysis of the microbial community was done using Quantitative Insights into Microbial Ecology (QIIME) and statistical analysis was inferred using $\mathrm{R}$.

\section{Results}

Alpha (within sample) diversity analysis showed the gut and oral microbiome to be more diverse than the vaginal microbiome. Significant differences in alpha diversity of the gut and oral microbiome between treatment groups was not confirmed. However, the vaginal microbiome of women with pre-eclampsia had a significant increase in evenness as observed with the Shannon Index (Kruskal Wallis chi-squared test: $\mathrm{P}=0.0472$ ). The evaluation of beta (between sample) diversity by principal coordinate analysis (PCOA) and the UniFrac distances revealed significant community differences of the vaginal microbiome in pre-eclampsia compared to normotensive pregnant women (Adonis: $P=0.05$ ). Lactobacillus iners was identified as the predominant species in the vaginal microbiome, however, a lower abundance of this species was detected in the pre-eclampsia group. Although the gut and oral microbiome did not reveal significant separation of these microbial communities in the pre-eclampsia group compared to normotensive pregnant women, a change in the relative abundance of several taxa was observed.

\section{Conclusion}

To our knowledge, this is the first study to investigate the diversity of the gut, oral and vaginal microbiome among pregnant women 
in South Africa with pre-eclampsia. Our results indicate an altered vaginal microbiome in pregnant women diagnosed with pre-eclampsia.

\section{ID: 8681}

Category: Clinical Microbiology (SASCM)

Permission: Yes

Co-Author 1: Ismail, $\mathrm{H}$

Co-Author 1 Institute: National Institute for Communicable Diseases Co-Author 2: Singh-Moodley, A

Co-Author 2 Institute: National Institute for Communicable Diseases Co-Author 3: van Schalkwyk, E

Co-Author 3 Institute: National Institute for Communicable Diseases

Co-Author 4: Candy, S

Co-Author 4 Institute: National Institute for Communicable Diseases

Co-Author 5: Perovic, 0

Co-Author 5 Institute: National Institute for Communicable Diseases

E-mail Address: ihusna@gmail.com

Country: South Africa

\section{ABSTRACT TITLE: EVALUATION OF PSEUDOMONAS AERUGINOSA ANTIMICROBIAL SUSCEPTIBILITY DATA OBTAINED FROM THE NHLS, LABORATORY INFORMATION SYSTEM WITH THE REFERENCE LABORATORY AT NICD, 2014 TO 2015, SOUTH AFRICA}

\section{Introduction}

Broth microdilution has been regarded as the reference method for antimicrobial susceptibility testing (AST) of Pseudomonas aeruginosa. However, the accuracy of automated AST systems, disk diffusion and Etest have become a concern. We aimed to compare AST results among clinical isolates of $P$. aeruginosa over a two-year period using data from a laboratory information system and the national reference laboratory.

\section{Methods}

We analysed data from patients admitted to 12 public sector sentinel hospitals from 2014 to 2015. Cases were identified through data extracts from the National Health Laboratory Service (NHLS), Corporate Data Warehouse (CDW) and the laboratory-based antimicrobial surveillance programme at the Centre for HealthcareAssociated Infections, Antimicrobial Resistance and Mycoses (CHARM). A case was defined as any patient with P. aeruginosa isolated from a blood culture specimen. A positive result obtained after 21 days of the first blood culture result was regarded as a new case. We compared categorical data (susceptible or non-susceptible including intermediate and resistant) for each method based on Clinical and Laboratory Standards Institute guidelines against six antimicrobial agents (cefepime, ceftazidime, colistin, imipenem, meropenem and piperacillin/tazobactam). Data from the NHLS, CDW contained AST data from the Vitek 2, MicroScan, disk diffusion or Etest and surveillance data from CHARM contained AST data for MicroScan and/or Sensititre.

\section{Results}

We assessed P. aeruginosa AST data for 1067 routine isolates and 571 surveillance isolates. Cefepime susceptibility was similar between NHLS (73\% [754/1038]) by NHLS and the reference laboratory (73\% [414/571]), meropenem susceptibility was observed in $72 \%(751 / 1046)$ by NHLS versus $69 \%(392 / 571)$ by the reference laboratory and piperacillin/tazobactam susceptibility was observed in $69 \%(706 / 1028)$ by NHLS versus $73 \%(414 / 571)$ by the reference laboratory.

\section{Conclusion}

Overall, we observed acceptable categorical agreements for AST from both routine NHLS laboratories and CHARM. We recommend that NHLS AST data for P. aeruginosa isolates can be used for reporting purposes.

\section{ID: 8265}

Category: Clinical Microbiology (SASCM)

Permission: Yes

Co-Author 1: Khillan, V

Co-Author 1 Institute: Institute of Liver and Biliary Sciences

Co-Author 2: Kale, $\mathrm{P}$

Co-Author 2 Institute: Institute of Liver and Biliary Sciences

E-mail Address: khillanv@yahoo.com

Country: South Africa

\section{ABSTRACT TITLE: PREVALENCE AND PROFILE OF TUBERCULOSIS IN LIVER DISEASES: ZILCH BUT EXCEPTIONS}

\section{Introduction}

Tuberculosis has a complex relationship with liver diseases, as the diseases may affect liver in disseminated cases with military disease, can be reactivated in immunosuppressive conditions or could affect the liver with toxic antitubercular therapy.

\section{Methods}

This study is the analysis of liver disease patients admitted to our tertiary care centre from January 2011 to December 2016.

Tuberculosis was diagnosed based on (i) histological evidence of caseating granulomas (ii) smear AFB positivity (iii) growth of Mycobacteria on MGIT culture (BD Microbiology Systems, Cockeysville, $\mathrm{MD}$ ) or (iv)positive $\mathrm{qPCR}$ for Mycobacterium tuberculosis (MTB qPCR) (Cobas TaqMan MTB assay). Mycobacteria other than tuberculosis (MOTT) were identified by positive MPT64 ICT assay (SD Bioloine) or MGIT culture positive and MTB qPCR negative samples.

\section{Results}

- Total no of samples: 816

- MGIT and or qPCR positive samples: 118 
- Pulmonary (sputum, ET secretions,BAL) 31 (26.2\%)

- Extra-pulmonary TB (asciticfluid, pus, bodyfluids, lymphnode aspirates, tissue, urine)

- 87 (73.7\%) Male 78/118 (66.1\%) Female $40 / 118$

- Median age. 44.5 years

\section{Conclusion}

- Our study reiterates these findings showing predominance of smear negative, extra-pulmonary TB and MOTT in our patients.

- MOTT accounting for $11 \%$ cases, should be ruled out in all cases as treatment varies with this group reducing hepatotoxicity by undue ATT exposure. MPT 64 assay is a rapid and easy method for detection of MOTT.

- A combination of MGIT culture and TB PCR has additive advantage over either test alone, with cumulative sensitivity of $100 \%$.

\section{ID: 8515}

Category: Clinical Microbiology (SASCM)

Permission: Yes

\section{Co-Author 1: Magobo, R}

Co-Author 1 Institute: University of Witwatersrand/ National Institute for Communicable Diseases - Centre for HealthcareAssociated Infections, Antimicrobial Resistance and Mycoses, a Division of the National Health Laboratory Services

Co-Author 2: Mhlanga, $M$

Co-Author 2 Institute: National Institute for Communicable Diseases - Centre for Healthcare-Associated Infections, Antimicrobial Resistance and Mycoses, a Division of the National Health Laboratory Services

Co-Author 3: Corcoran, C

Co-Author 3 Institute: Ampath National Reference Laboratory

Co-Author 4: Thomas, J

Co-Author 4 Institute: National Institute for Communicable Diseases - Centre for Healthcare-Associated Infections, Antimicrobial Resistance and Mycoses, a Division of the National Health Laboratory Services

Co-Author 5: Govind, C

Co-Author 5 Institute: Lancet Laboratories

Co-Author 6: Lowman, W

Co-Author 6 Institute: Pathcare, Cape Town

Co-Author 7: Senekal, M

Co-Author 7 Institute: Pathcare, Cape Town

Co-Author 8: Govender, NP

Co-Author 8 Institute: University of Witwatersrand and National Institute for Communicable Diseases - Centre for HealthcareAssociated Infections, Antimicrobial Resistance and Mycoses, a Division of the National Health Laboratory Services

E-mail Address: Rindidzani.magobo2@wits.ac.za

Country: South Africa
ABSTRACT TITLE: THE EPIDEMIOLOGY OF CANDIDA AURIS IN SOUTH AFRICA, 2012-2016

\section{Background}

We sought to describe the epidemiology and genetic relatedness of Candida auris, an emerging multidrug-resistant invasive fungal pathogen associated with high mortality, in South Africa.

\section{Methods}

Culture-confirmed cases of C. auris infection/colonization were reported 2012-2016 by private- and public-sector laboratories across South Africa. We defined a case as a patient with culture of C. auris (or C. haemulonii if the diagnostic laboratory used a nonconfirmatory method) from any specimen. For an isolate subset with confirmed molecular identification, we performed antifungal susceptibility testing, sequencing of the FKS1 and FKS2 genes (if echinocandin MIC $\geq 1 \mathrm{mg} / \mathrm{L}$ ) and multi-locus sequence typing (MLST).

\section{Results}

1695 cases of C. auris were reported, 1406 (88\%) from Gauteng province. The number of cases increased from 4 in 2012-2013 to 380 in 2015-2016. Ninety-three per cent (1545/1659) of patients were admitted to private-sector hospitals. The median age was 59 years (IQR: 42-72) and 62\% (1029/1652) were male. Overall 739 (47\%) isolates were cultured from sterile site specimens (blood [344, 22\%], cerebrospinal fluid $[2,0.1 \%]$, fluid [56, 4\%], tissue [49, 3\%]), central venous catheter tips [288, 18\%] and $840(53 \%)$ were cultured from sites of potential colonization (urine [622,39\%], respiratory tract $[173,11 \%]$, skin/mucosal swabs [45, 3\%]). Of 85 isolates confirmed as C. auris, the MIC 50 (mg/L) and MIC 90 (mg/L) for fluconazole, voriconazole, micafungin and amphotericin $B$ was 128, 256; $0.12,1 ; 0.06,2$ and $0.5,1$ respectively. No known FKS mutations were detected. MLST analysis grouped isolates into two clusters comprising of 83 and 2 isolates respectively.

\section{Conclusion}

There was a large increase in reported cases of $\mathrm{C}$. auris since its first isolation in South Africa in 2012. C. auris strains were mostly fluconazole non-susceptible and highly related by MLST though the possibility of nosocomial transmission should be explored using more discriminatory whole genome sequencing.

\section{ID: 8378}

Category: Clinical Microbiology (SASCM)

Permission: Yes

Author: Maluleka, C

Author Institute: National Health Laboratory Service, Dr George Mukhari Academic Hospital, Sefako Makgatho Health Sciences University

E-mail Address: caroline.maluleka@nhls.ac.za

Country: South Africa 
ABSTRACT TITLE: PREVALENCE AND SUSCEPTIBILITY PROFILE OF ACINETOBACTER BAUMANII ISOLATED AT HIGH CARE WARDS AT DR GEORGE MUKHARI ACADEMIC HOSPITAL IN 2015

\section{Introduction}

Antimicrobial resistance is a general public health concern, threatening the effective management and Infection control of infections. Globally, Acinetobacter baumanii has been reported as the major pathogen isolated in hospitals with high resistance to major antimicrobial agents and thus termed Multidrug resistant organisms. The organism results in high morbidity and mortality because of limited treatment options. The aim of the study was to determine the prevalence and antimicrobial susceptibility profile of $A$. baumanii isolated from blood culture specimens taken at selected high care wards of Doctor George Mukhari Academic Hospital in 2015.

\section{Methods}

This was a retrospective descriptive study. National Health Laboratory Services (NHLS) laboratory information system (TrakCare) was used to obtain data on A. baumanii isolates isolated from blood culture specimens in 2015 using Microsoft Excel. Only blood culture results from high care wards $(6,9,20,24,39$ and ICU) were filtered, repeats excluded and data on the susceptibility trends to select antimicrobial agents was analysed.

\section{Results}

A total of 630 blood culture results were retrieved during the study period. Sixty-five $(10,3 \%)$ of the isolates were identified as A. baumanii. A. baumanii was more prevalent in ICU at 44,6\%(29/65). The organisms showed resistance to majority of the antimicrobials tested. They exhibited resistance to aminoglycosides; with Amikacin at $29 \%$, and gentamicin at $78 \%$. Most of the isolates also exhibited resistance to carbapenems: Imipenem and meropenem, at $88 \%$ and $90 \%$ respectively. Fortunately, all the isolates were susceptible to collistin.

\section{Conclusion}

High level of resistance to carbapenems is worrisome since these are the initial treatment options for patients admitted in high care areas. It also poses a threat to effective management and infection prevention and control measures in our setting.

\section{ID: 8512}

Category: Clinical Microbiology (SASCM)

Permission: Yes

Co-Author 1: Mogale, $R$

Co-Author 1 Institute: University of Pretoria

Co-Author 2: Ehlers, M

Co-Author 2 Institute: University of Pretoria, National Health Laboratory Services

E-mail Address: remomog@gmail.com

Country: South Africa

\section{ABSTRACT TITLE: MORPHOLOGICAL DIVERSITY OF PANTON-VALENTINE LEUKOCIDINE POSITIVE STAPHYLOCOCCUS AUREUS BACTERIOPHAGES IDENTIFIED IN SOUTH AFRICA AND NIGERIA}

\section{Introduction}

Panton-Valentine leukocidine (PVL) is a bacteriophage-encoded bi-component, pore-forming leukotoxin, causing leukotoxin lysis. The presence of the PVL gene in Staphylococcus aureus has been associated with severe necrotising pneumonia and necrotising fasciitis. Ten bacteriophage types belonging to three morphologically distinct head-groups have been reported to aid in the horizontal gene transfer (HGT) of PVL genes. The aim of this study was to identify the morphological diversity of PVL positive S. aureus bacteriophages from South Africa and Nigeria

\section{Methods}

A total of 70 previously stored isolates consisting of MRSA and MSSA isolates from South Africa and Nigeria were collected from the culture bank in the Department of Medical Microbiology, University of Pretoria/National Health Laboratory Services (NHLS). The presence of the genus (16S rRNA), species specific (nuc), methicillin-resistance $(\mathrm{mec} A)$ and the PVL genes were confirmed using Multiplex-PCR (M-PCR) assays.Multiplex-PCR assays were performed to identify the morphological diversity of bacteriophage types within the $S$. aureus isolates.

\section{Results}

The species confirmation M-PCR assay correlates with previous analysis performed on these isolates. The results indicated the icosahedral head-group II [72.6\% (53/70)] was the most prevalent head-group, followed by the elongated head-group [70\% (49/70)] and icosahedral head-group I [28.5\% (20/70)]. All three bacteriophage head-group morphologies were detected in 5.7\% (4/70) MSSA and 2.8\% (2/70) MRSA isolates from South Africa and only one MRSA isolate from Nigeria.

\section{Conclusion}

While the elongated head-group has been reported as the most prevalent in Italy, an equal distribution of bacteriophage morphology has been reported in China. This is in contrast with the results found in this study. The high prevalence of the icosahedral head-group II bacteriophages carrying the PVL genes, suggests geographic variation according to phage-lineage types and that these bacteriophages may be easily transferred to $S$. aureus isolates in clinical settings of the study population under investigation.

\section{ID: 8517}

Category: Clinical Microbiology (SASCM)

Permission: Yes

Co-Author 1: Ndlovu, S

Co-Author 1 Institute: University of Pretoria

Co-Author 2: Mbelle, NM 
Co-Author 2 Institute: University of Pretoria, National Health Laboratory Service

Co-Author 3: Malinga, LA

Co-Author 3 Institute: Medical Research Council

Co-Author 4: Fourie, B

Co-Author 4 Institute: University of Pretoria

E-mail Address: Ndlovusamkelisiwe2@gmail.com

Country: South Africa

\section{ABSTRACT TITLE: EVALUATION OF GENOTYPE ${ }^{\circledR}$ MTBDRSL (VER 2.0) AGAINST SECOND-LINE DRUG SUSCEPTIBILITY TESTING AND IMPACT OF GYRA MUTATIONS ON FLUOROQUINOLONE SUSCEPTIBILITIES}

\section{Introduction}

The emergence of Mycobacterium tuberculosis resistant strains is threatening the control of multidrug resistant tuberculosis (MDRTB) worldwide. To curb the spread, fluoroquinolones (FLQ) and second line injectable drugs (SLIDs) are used to treat MDR-TB. A recently endorsed, GenoType ${ }^{\oplus}$ MTBDRsl is used for rapid detection of resistance towards FLQ and SLIDs and guide treatment options for patients while awaiting phenotypic drug susceptibility testing (DST). The aim of the study was to evaluate Genotype ${ }^{\oplus}$ MTBDRsl against DST and correlate the phenotypic results with mutations.

\section{Methods}

We collected MDR-TB isolates $(n=54)$ from the National Health Laboratory Service, Pretoria. All isolates were sub-cultured, and DST was performed on the MGIT 960 system. The critical concentrations (CC) used were $2.0 \mu \mathrm{g} / \mathrm{ml}$ for Ofloxacin (OFX), 0.5 and $2.0 \mu \mathrm{g} / \mathrm{ml}$ for Moxifloxacin (MOX), $1.0 \mu \mathrm{g} / \mathrm{ml}$ for amikacin (AMK), $2.5 \mu \mathrm{g} / \mathrm{ml}$ for kanamycin (KAN) and capreomycin (CAP) drugs. GenoType ${ }^{\odot}$ MTBDRslassay was performed according to manufacturer (Hain LifeScience, Germany) and compared to DST.

\section{Results}

Sensitivity of the GenoType ${ }^{\oplus}$ MTBDRslassay was $100 \%$ for OFX, MOX $(0.5 \mu \mathrm{g} / \mathrm{ml})$ and MOX $(2.0 \mu \mathrm{g} / \mathrm{ml})$ with specificity of $100 \%$, $91.84 \%$ and $88.24 \%$ respectively. Sensitivity and specificity for KAN, AMK and CAP were $75 \%$ and $100 \%, 75 \%$ and $100 \%$ and $50 \%$ and $98 \%$ respectively. Mutations of A90V resulted mostly in low level MOX resistance $(<0.5 \mu \mathrm{g} / \mathrm{ml})$ as compared to D94G with high level resistance $(>0.5 \mu \mathrm{g} / \mathrm{ml})$. Mutations of A1401G $(n=2)$ were detected in AMK/KAN/CAP resistant cases, while G1484T $(n=1)$ was in AMK/ KAN case.

\section{Conclusion}

GenoType ${ }^{\oplus}$ MTBDRslassay is a useful tool and has excellent sensitivity for FLQ resistance, but moderate to high specificity for MOX and OFX respectively. Rapid detection of different mutations can guide clinicians in prescribing appropriate drugs while awaiting DST. More studies are needed, to improve the accuracy SLID rapid detection.

\section{ID: 8293}

Category: Clinical Microbiology (SASCM)

Permission: Yes

Co-Author 1: Tshisevhe, V

Co-Author 1 Institute: National Health Laboratory Services, University of Pretoria

Co-Author 2: Onwuegbuna, 0

Co-Author 2 Institute: National Health Laboratory Services

Co-Author 3: Mbelle, N

Co-Author 3 Institute: National Health Laboratory Services, University of Pretoria

E-mail Address: vhudzani@gmail.com

Country: South Africa

\section{ABSTRACT TITLE: COMPARISON OF COMMERCIAL MPT64AG-BASED ASSAYS FOR RAPID IDENTIFICATION OF MYCOBACTERIUM TUBERCULOSIS IN CLINICAL ISOLATES}

\section{Introduction}

The increase in cases of Mycobacterium tuberculosis (TB) infections as reported by WHO Global TB report 2015, necessitate rapid detection of cases to facilitate implementation of appropriate infection prevention and control measures together with timely initiation of treatment.

Studies have shown that MPT64 based assays have high sensitivity and specificity for identification of MTB. Furthermore, these assays have rapid turnaround time. We compared the Hains MPT64 antigen assay test to BD MGIT TBC Identification Test (TbC ID), cord formation and the Genotype Mycobacterium CM molecular assay.

\section{Methods}

Archived samples received for routine diagnostic testing between May and October 2014 were used. All clinical isolates that flagged positive on MGIT had Ziehl Nielsen performed to check cord formation, antigen tests done, and molecular test performed.

\section{Results}

A total of 29 isolates were cultured from 20 sputum, 8 tissue and 1 CSF specimens. Cord formation was observed in 19 specimens. The molecular assay was positive for MTB in all the isolates including the 6 BCG isolates. BD MGIT TBC ID was positive in 17 of these isolates. The TBC ID showed PPV of $0.74(0.49-0.91)$ and NPV of 0.56 $(0.21-0.86)$, and a PPV of $0.86(0.64-0.97)$ and NPV $1(0.54-1.00)$. The Hains assay was positive for all isolates.

\section{Conclusion}

The good performance of the Hains MPT64 antigen assay compared to the molecular test means it can be used to replace the old MPT64 based assays such as the TBC ID that have poor negative predictive values. 


\section{ID: 8431}

Category: Clinical Microbiology (SASCM)

Permission: Yes

Co-Author 1: Perovic, $\mathrm{O}$

Co-Author 1 Institute: Centre for Healthcare-Associated Infections, Antimicrobial Resistance and Mycoses, National Institute for Communicable Diseases and University of Witwatersrand

Co-Author 2: Singh-Moodley, A

Co-Author 2 Institute: Centre for Healthcare-Associated Infections, Antimicrobial Resistance and Mycoses, National Institute for Communicable Diseases and University of Witwatersrand

Co-Author 3: van Schalkwyk, E

Co-Author 3 Institute: Centre for Healthcare-Associated Infections, Antimicrobial Resistance and Mycoses, National Institute for Communicable Diseases

\section{E-mail Address: olga.perovic@nhls.ac.za}

Country: South Africa

\section{ABSTRACT TITLE: CHANGES IN SUSCEPTIBILITY PATTERN TO OXACILLIN IN STAPHYLOCOCCUS AUREUS ISOLATES FROM PATIENTS WITH BLOODSTREAM INFECTIONS IN SOUTH AFRICA OVER A SEVEN -YEAR PERIOD}

\section{Introduction}

This report aimed to describe antimicrobial susceptibility and distribution of Staphylococcus aureusbacteraemia (SAB) isolates among hospitalised patients in South Africa over a seven-year period. We also aimed to determine the molecular characteristics of methicillin-resistant S. aureus (MRSA).

\section{Methods}

The study population included patients of all ages accessing publicsector healthcare. All isolates from patients with SAB were submitted for confirmatory identification and antimicrobial susceptibility testing for the Laboratory-based Antimicrobial Resistance Surveillance (LARS) programme periodically. Susceptibility testing was performed on the MicroScan Walkaway system (Siemens Healthcare Diagnostics, USA) using the Positive MIC Panel Type 33. The LightCycler 480 II instrument (Roche Applied Science, Germany) was used for the real-time PCR amplification of the methicillin resistance determinant, mecA.

\section{Results}

A total of 5358 patients with SAB were identified from June 2010 to June 2017. Among 5322 of these cases with a viable isolate, confirmed molecular identification and full susceptibility profile, 1967 (35\%) were classified as MRSA and confirmed with mecA. The ratio of MRSA to methicillin-susceptible $S$. aureus (MSSA) cases decreased from $51 \%$ (286/558) in 2010 to $25 \%$ (77/311) in 2017. We analysed 1967 isolates resistant to oxacillin and confirmed as MRSA due to the presence of mecA. The most common SCCmec type was SCCmec type III ( $n=860,43 \%$ ) followed by type IV ( $n=609,30 \%)$. Spatyping of the 1149 oxacillin-resistant isolates revealed the five most common spa-types were t037 ( $n=556,30 \%), t 1257(n=214,11 \%)$ t045 ( $n=95,5 \%)$, t012 $(n=58,3 \%)$ and t064 $(n=46,2 \%)$. MLST showed the most common ST to be ST612 (CC8) followed by ST239 of clonal complex (CC8).

\section{Conclusion}

We found a decrease in MRSA over the seven-year period. SCCmec type III predominated; however, the presence of SCCmec type IV has become evident, indicating that this type is emerging in hospitals.

\section{ID: 8445}

Category: Clinical Microbiology (SASCM)

Permission: Yes

Co-Author 1: Sandeep, V

Co-Author 1 Institute: Walter Sisulu University

Co-Author 2: Obi, L

Co-Author 2 Institute: University of Fort Hare

E-mail Address: sandeepvasaikar@yahoo.com

Country: South Africa

ABSTRACT TITLE: SPECIFIC DETECTION OF LABORATORY MISIDENTIFIED KLEBSIELLA VARIICOLA BY SANGER SEQUENCING FROM KNEE EFFUSION

\section{Introduction}

K. variicola was described as a new bacterial species in 2004, based on the phylogenetic analysis of six housekeeping (rpoB, gyrA, nifH, infB, phoE and $\mathrm{mdh}$ ) genes. It is found mostly from plant and insects and only bloodstream infections. To date, the correct identification of Klebsiella species has not been easily achieved in microbiological laboratories because several species of this genus share similar biochemical profiles. We report a case of the female patient from Easter Cape with left knee effusion from whom K. variicola was misidentified as K. pneumoniae by automated method.

\section{Case}

A 44-year-old female patient from ECP with left knee effusion was admitted to the St. Elizabeth Hospital in Lusikisiki. Sterile fluid specimen, gram-negative bacilli. On overnight incubation, lactose fermenting colonies resembling Klebsiella species were isolated, which were identified as K. pneumoniae by B \& C AutoSCAN using NID2 panel with $96 \%$ probability. MIC was determined by B\&D Type 37 panel, which demonstrated sensitive to most antibiotics except ampicillin and ESBL -ve. DNA of the isolate was sequenced by the Sanger method at the Inqaba BioTech Lab, using an Applied Biosystems 3500xL Genetic Analyser. MLST typing was done which identified this isolate as K. variicola (matching with $\mathrm{K}$. variicola DSM $15968 \mathrm{NCBI}$ ) which was previously identified as K. pneumoniaeby pheneotypic method.

\section{Discussion}

K. variicola, a bacterium closely genetically related to K. pneumoniae, is commonly misidentified as K. pneumoniae by biochemical tests. It is mostly isolated from environmental sources such as plants 
and insects (termites) and animals (gorilla). Misidentification of Klebsiella species by automated systems has been reported by Berry et. al. S. K. variicola seems to have lower antibiotic resistance as compared to K. pneumoniae but bloodstream infections have higher mortality rate. No additional virulence factors were found for increased deaths by Maatallah et al. Our isolate was ESBL negative and sensitive to almost all antibiotics except ampicillin, but we found presence of blaCTX-M.

\section{ID: 8454}

Category: Clinical Microbiology (SASCM)

Permission: Yes

Co-Author 1: Schnugh, D

Co-Author 1 Institute: National Health Laboratory Service, University if the Witwatersrand

Co-Author 2: Thomas, $T$

Co-Author 2 Institute: National Health Laboratory Service

Co-Author 3: Duse, A

Co-Author 3 Institute: National Health Laboratory Service, University if the Witwatersrand

E-mail Address: desmond.schnugh@nhls.ac.za

Country: South Africa

\section{ABSTRACT TITLE: A TWO-YEAR STUDY OF THE INCIDENCE OF NOCARDIA}

\section{Introduction}

Nocardia is a gram-positive organism found predominately in soil and the oral microflora of humans. Nocardia has been linked to a wide range of human disease. Taking into account the high level of immune compromised individuals in South Africa due to HIV infection, it would be expected to see a high level of these infections.

This study aimed to investigate the incidence of Nocardia with respect to patient age, HIV status, type of referring ward, species identified and antibiotic susceptibility in state hospitals in Gauteng over a 2-year period.

\section{Methods}

Samples sent to the Infection Control Services Laboratory, NHLS, for bacterial identification and antibiotic susceptibility results for the period 01/042015 to 30/072017 were included in the study. The genus and species of the isolates were identified through $16 \mathrm{~S}$ rRNA sequencing. Antibiotic susceptibility testing was performed by the manual broth dilution method.

\section{Results}

36 isolates were sequenced during this period. The main Nocardia species identified were N.arthritidis and N.exalbida. 12 different species of Nocardia were identified in the study. The average age at presentation was 37.3 years. The main referral wards were the surgical and medical wards. The main type of specimen was fluid from an abscess. The HIV status of only $47 \%$ of the patients were known of which all were HIV infected. The mean viral load was
52919. All but one isolate was sensitive to Cotrimoxazole. However, it was not possible to speciate this isolate.

\section{Conclusion}

The numbers of Nocardia in this study are an underestimate of the scale of disease because 1. many patients are miss diagnosed as TB and 2. this study is only covering isolates referred to our lab for testing. However, the study does provide some data on Nocardia species seen in the Gauteng setting since the last study in 2010.

\section{ID: 8284}

Category: Clinical Microbiology (SASCM)

Permission: Yes

Co-Author 1: Hoffmann, R

Co-Author 1 Institute: University of Stellenbosch, National Health Laboratory Service

Co-Author 2: Singh, S

Co-Author 2 Institute: National Health Laboratory Service

E-mail Address: renah@sun.ac.za

Country: South Africa

ABSTRACT TITLE: EARLY-ONSET NEONATAL

STREPTOCOCCUS GALLOLYTICUS SUBSPECIES PASTEURIANUS MENINGITIS AND SEPTICEMIA

\section{Introduction}

Streptococcus gallolyticus subspecies pasteurianusis a highly unusual pathogen in neonates. We describe a case of this organism causing early-onset meningitis and septicaemia.

\section{Case}

A HIV-exposed premature infant presented on day 1 of life with respiratory distress and shock; complicated by jejunal atresia repaired on day 3. Intravenous ampicillin and gentamycin were initiated after blood cultures and a septic screen were performed. Streptococcus gallolyticus subsp. pasteurianus was isolated from a blood culture. Cerebrospinal fluid (CSF) collected on day 3 of life confirmed a diagnosis of meningitis. Bacteria were not cultured from the CSF, however, a Polymerase Chain Reaction (PCR) performed directly on the CSF demonstrated the presence of Streptococcus gallolyticus subsp. pasteurianus. The patient was treated in the neonatal ICU with 14 days of ampicillin. No neurological complications at discharge were noted.

\section{Discussion}

Streptococcus gallolyticus subsp. pasteurianus has replaced Streptococcus bovis II/2; part of group D noneterococcal streptococci.

Bacteremia is the most common clinical manifestation of early-onset S. bovis group infection. Neonates with early-onset $\mathrm{S}$. bovisgroup bacteremia generally present with acute onset of respiratory distress and sepsis within the first 5 days of life. In contrast, lateonset $\mathrm{S}$. bovis group infection generally presents with urinary sepsis or meningitis. 
Streptococcus gallolyticus subsp. pasteurianus is capable of causing fulminant neonatal sepsis or meningitis that is clinically indistinguishable from that caused by group B streptococcus. Brain abscesses, delayed subdural effusions and brain haemorrhages have been described as complications of this infection and therefore long- term neurological follow-up of these infants are recommended.

\section{ID: 8300}

Category: Clinical Microbiology (SASCM)

Permission: Yes

Co-Author 1: Singh-Moodley, A

Co-Author 1 Institute: National Institute for Communicable Diseases

Co-Author 2: Perovic, $\mathrm{O}$

Co-Author 2 Institute: National Institute for Communicable Diseases

E-mail Address: ashikas@nicd.ac.za

Country: South Africa

\section{ABSTRACT TITLE: PHENTOYPIC AND GENOTYPIC CORRELATION OF CARBAPENEMEMASE-PRODUCING ENTEROBACTERIACEAE AND RECOMMENDATIONS FOR THE ROUTINE SCREENING OF CARBAPENEM-RESISTANT ENTEROBACTERIACEAE}

\section{Introduction}

The emergence and transmission of CRE is concerning in the clinical and public health arena. Reliable and accurate detection for patient management and infection prevention and control purposes is required. Routinely, phenotypic methods are utilised for identification of CRE.

\section{Methods}

Phenotypic profiles of 2678 suspected-CPE isolates generated by the automated MicroScan ${ }^{\circledast}$-Walkaway-system using CLSI guidelines were correlated with carbapenemase production as identified molecularly.

\section{Results}

Klebsiella pneumoniae accounted for $63 \%$ of all isolates ( $n=1685$ ) followed by Enterobacter cloacae $(n=361,14 \%)$. Carbapenemases accounted for $75 \%$ of isolates (blaOXA-48 ( $n=978,37 \%)$, blaNDM ( $n=904,34 \%)$, blaVIM $(n=108,4 \%)$, blaIMP $(n=35,1.3 \%)$, blaGES $(\mathrm{n}=24,0.9 \%)$, blaKPC $(\mathrm{n}=18,0.7 \%))$.A substantial number of isolates expressing a carbapenemase/s were susceptible to third-and fourth-generation cephalosporins and carbapenems demonstrating that confirmed carbapenemase-producing isolates are not presenting as possible carriers of carbapenemases using routine diagnostic methods. Similar results were obtained when CLSI and EUCAST clinical breakpoints were applied. This was improved using EUCAST epidemiological cut-off (ECOFF) values. The recommended ECOFF value of $>0.12 \mathrm{mg} / \mathrm{mL}$ for meropenem, reduced the number of missed carbapemase-producing isolates. Exact quantification could not be performed as dilutions lower than the lowest MIC dilution is required for analysis.

\section{Conclusion}

While ECOFF values are appropriate for infection prevention and control purposes, clinical breakpoints are suitable for patient management. Considering this, and other published data investigating appropriate methods for carbapenemase screening, routine laboratories should use a combination of the EUCAST meropenem screening cut-off of $<25 \mathrm{~mm}$ or MIC $>0.12 \mathrm{mg} / \mathrm{ML}$ and ertapenem (or meropenem) APBA and EDTA combination disk tests plus temocillin disk diffusion on $\mathrm{MH}-\mathrm{CLX}$ agar.

\section{ID: 8485}

Category: Clinical Microbiology (SASCM)

Permission: Yes

Co-Author 1: Strasheim, W

Co-Author 1 Institute: National Institute for Communicable Diseases

Co-Author 2: Singh-Moodley, A

Co-Author 2 Institute: National Institute for Communicable Diseases

Co-Author 3: Perovic, 0

Co-Author 3 Institute: National Institute for Communicable Diseases

E-mail Address: wilhelminas@nicd.ac.za

Country: South Africa

\section{ABSTRACT TITLE: PREDOMINANT METHICILLIN- RESISTANT STAPHYLOCOCCUS AUREUS SPA-TYPES CIRCULATING AT FIVE SENTINEL SITES ACROSS SOUTH AFRICA}

\section{Introduction}

Staphylococcal protein A (spa)-typing is a valuable tool to track the spread of Staphylococcus aureus. Spa-typing exploits S. aureus' clonal nature and therefore isolates with the same spa-type are related. The aim of this study was to define the predominant methicillin-resistant $\mathrm{S}$. aureus (MRSA) spa-types circulating at five sentinel sites across South Africa.

\section{Methods}

Staphylococcus aureus bloodstream infection isolates were submitted to the national reference laboratory as part of GERMS surveillance from 2010-2015. Methicillin-resistance was defined as the PCR detection of the mecA gene and non-susceptibility to oxacillin and/or cefoxitin. Molecular testing included spa-typing, Staphylococcal Chromosome Cassette (SCC) mec typing and multilocus sequencing typing (MLST) on selected isolates.

\section{Results}

A total of 3167 isolates were submitted and 34\% (1087/3167) were methicillin-resistant, of which $74 \%$ underwent spa-typing. Spa-type t037 predominated in three Gauteng hospitals [Hospital A $=73 \%$ (203/278); Hospital B = 47\% (52/110) and Hospital C = 50\% (56/113)] and in a Western Cape hospital [Hospital E = 27.3\% (44/161)]. Spatype t1257 $(27 \%, 37 / 139)$ predominated in another Western Cape hospital. The majority of t037 cases in the Western Cape occurred in 
a burn unit (59\%,26/44) among adult males (85\%, 22/26). Most t037 cases in Gauteng hospitals were associated with paediatric patients residing in a specific ward within the respective hospital [Hospital $A$ $=28 \%(57 / 203)$ in a premature ward; Hospital $B=60 \%(31 / 52)$ in a paediatric ICU and Hospital C $=25 \%$ (14/56) in a paediatric surgery ward]. Most t037 isolates in all hospitals harboured SCCmec type III $(93 \%, 330 / 355)$ and three isolates belonged to MLST clonal complex (CC) 8, sequence type (ST) 239.

\section{Conclusion}

The predominant spa-types in South Africa are t037 and t1257. Preliminary results show that isolates are possibly related and that further investigation with pulsed-field gel electrophoresis is warranted to assist in infection prevention and control efforts.

\section{ID: 8447}

Category: Paediatric Infectious Diseases (SASPID)

Permission: Yes

Co-Author 1: Kgasha, 0

Co-Author 1 Institute: Sefako Makgatho Health Science University

Co-Author 2: Maluleka, C

Co-Author 2 Institute: Sefako Makgatho Health Science University, National Health Laboratory Services

Co-Author 3: Rahulani, L

Co-Author 3 Institute: University of the Witwatersrand

E-mail Address: olga.kgasha@smu.ac.za

Country: South Africa

ABSTRACT TITLE: AN INVESTIGATION OF STREPTOCOCCUS PNEUMONIAE COLONISING THE NASOPHARYNX OF CHILDREN ATTENDING THE PAEDIATRIC CLINIC AT

DR. GEORGE MUKHARI ACADEMIC HOSPITAL

\section{Introduction}

Polysaccharide Conjugate Vaccine (PCV) was introduced in 2009 in the South African childhood immunisation programme. Since then, there has been a dramatic decrease in the incidence of pneumococcal diseases in both vaccinated children and nonvaccinated individuals of all ages. However, increased infections caused by non-vaccine serotypes have been reported. The reduction in vaccine serotypes may however open a niche in the nasopharynx allowing for increases in the acquisition and prevalence of pneumococcal non-vaccine serotype colonization and subsequent disease. Following the South African PCV introduction, the impact of PCV on nasopharyngeal colonization has not been adequately assessed. Therefore, the aim of the study was to investigate the prevalence of $\mathrm{S}$. pneumoniae colonising the nasopharynx of children aged 2 months-14yrs attending the paediatric clinic at Dr. George Mukhari Academic Hospital.

\section{Methods}

Collected nasopharyngeal swabs were cultured. Isolates were identified phenotypically and were serotyped using conventional multiplex PCR targeting 29 of the most invasive serotypes and those included in the PCV.

\section{Results}

Thirteen-percent (47/350) of the isolates were positive for S. pneumoniae. Forty two percent (42\%) of the isolates were serotyped using PCR. Identified serotypes were $6 A / B(35 \%), 1(25 \%)$, 7C (25), 8(5\%), 31(5\%) and 20(5\%).

\section{Conclusion}

Non-vaccine serotypes detected in this study are an indication of serotype replacement, with the decline of vaccine serotypes giving way for non-vaccine serotypes to prevail. Overall, PCV has shown alteration in colonising serotypes.

\section{ID: 8392}

Category: Clinical Microbiology (SASCM)

Permission: No

Co-Author 1: Tootla, H

Co-Author 1 Institute: National Health Laboratory Service, University of Cape Town

Co-Author 2: Moodley, C

Co-Author 2 Institute: National Health Laboratory Service, University of Cape Town

Co-Author 3: Bamford, C

Co-Author 3 Institute: National Health Laboratory Service, University of Cape Town

E-mail Address: Hafsah.tootla@gmail.com

Country: South Africa

\section{ABSTRACT TITLE: THE USE OF A URINARY ANTIGEN TEST FOR THE RAPID IDENTIFICATION OF S. PNEUMONIAE BACTERAEMIA}

\section{Introduction}

Novel detection methods for invasive pneumococcal disease (IPD) include PCR and antigen tests. The 'gold standard' for diagnosis of IPD is culture from sterile sites. Prior antibiotic therapy, autolysin production and the fastidious nature of pneumococci can yield culture negative results. The aim of this study was to evaluate the use of a novel urine antigen test directly on blood cultures, for the rapid identification of $S$. pneumoniae.

\section{Methods}

The BinaxNOW Streptococcus pneumoniae urinary antigen test was compared to routine culture with optochin, and lytA PCR, on blood cultures with Gram positive cocci in chains or pairs, and where the morphology was indiscriminate. 


\section{Results}

Preliminary results of 46 blood cultures tested indicated that 5 were non-viable on culture. When compared to culture, the sensitivity (7/7) and specificity (34/34) of the antigen test was $100 \%$. The 5 non-viable samples were however, positive on both PCR and the antigen test.

When comparing the antigen test to PCR, the sensitivity was $75 \%$ (12/16), and specificity was $100 \%$ (30/30). Of the 16 lytA positive samples, the antigen test reported 4 as negative. These isolates were other Gram-positive cocci on culture, and produced high $\mathrm{CT}$ values on PCR. When excluded from analysis, the sensitivity and specificity of the test increased to $100 \%$.

\section{Conclusion}

The use of the urine antigen test on blood culture, in conjunction with Gram stain, gave a rapid and accurate presumptive diagnosis of IPD. This would allow for targeted antimicrobial therapy sooner, and promote antibiotic stewardship. It can also be used as a costeffective alternative to molecular testing in cases of culture negative S. pneumoniae bacteraemia.

\section{ID: 8514}

Category: Paediatric Infectious Diseases (SASPID)

Permission: No

Author: Khosa, X

Author Institute: Sefako Makgatho Health Sciences University

E-mail Address: caroline.maluleka@nhls.ac.za

Country: South Africa

\section{ABSTRACT TITLE: MOLECULAR CHARACTERISATION AND ANTIMICROBIAL SUSCEPTIBILITY TESTING OF GROUP A STREPTOCOCCUS ISOLATES FROM INVASIVE AND NON- INVASIVE INFECTIONS AT PHEDISONG 4, SOSHANGUVE 3, AND DR GEORGE MUKHARI ACADEMIC HOSPITAL}

\section{Introduction}

Group A streptococcus (GAS) is responsible for a wide range of invasive and non-invasive infections. Pharyngitis due to GAS may cause complications such as acute rheumatic fever/ rheumatic heart disease which have high mortality and morbidity rates in developing countries. Antimicrobial susceptibility testing and molecular characterisation of GAS isolates will yield information required for treatment using antibiotics and preventative vaccine development

\section{Aim}

The study aims to determine the emm genotypes and antimicrobial susceptibility profiles of GAS isolates circulating in the North-West area of Pretoria.

\section{Methods}

This is a quantitative cross-sectional study describing the prevalent emm genotypes circulating in the region. Throat swabs from patients presenting with pharyngitis from the clinics were collected for GAS isolation. GAS isolates were also collected from the National Health Laboratory Services (NHLS). Quantitative antimicrobial susceptibility testing was done for Penicillin, Clindamycin, Erythromycin and disk susceptibility testing for Cotrimoxazole. Genotyping of GAS strains will be done using PCR, sequencing and pulsed field gel electrophoresis.

\section{Results}

A total of 24 samples were collected, from which, nine were throat swabs and 17 were GAS clinical isolates from the NHLS. From the nine collected swabs, two were identified as GAS. MICs for the Penicillin, Clindamycin, Erythromycin ranged from 0.012-0.08 ug/ml, $\quad 0.016-0.125 \mathrm{ug} / \mathrm{ml}, \quad 0.016-0.064 \mathrm{ug} / \mathrm{ml}$ respectively. Zone sizes for cotrimoxazole were between 20-35mm which were also susceptible

The MICs for the antimicrobials tested were all at the lower ranges of susceptibility breakpoints.

\section{Conclusion}

GAS isolates still remain susceptible to penicillin, despite reports of macrolide resistance; all of our isolates were susceptible and were inducible Clindamycin resistance negative. Molecular characterisation of the emm types is ongoing.

\section{ID: 8240}

Category: Paediatric Infectious Diseases (SASPID)

Permission: Yes

Co-Author 1: Nyamurenje, L

Co-Author 1 Institute: University of KwaZulu Natal

Co-Author 2: Archary, M

Co-Author 2 Institute: University of KwaZulu Natal

E-mail Address: Inyamurenje@gmail.com

Country: South Africa

\section{ABSTRACT TITLE: CULTURE CONFIRMED BACTERIAL INFECTIONS IN HIV-INFECTED AND UNINFECTED SEVERELY MALNOURISHED CHILDREN ADMITTED TO KING EDWARD VIII HOSPITAL, DURBAN}

\section{Introduction}

Malnutrition results in an alteration in both innate and adaptive host defence mechanisms, resulting in an increased susceptibility to infections. This study seeks to describe and compare culture confirmed bacterial infections in HIV negative severely malnutrition with those from HIV positive patients admitted in the same setting.

\section{Methods}

Specimens (blood, cerebrospinal fluid, urine and sputum) obtained from 101 children aged between six and sixty months admitted with severe acute malnutrition (SAM) in King Edward Hospital Durban South Africa between 1 January 2015 and 31 December 2015 were retrospectively identified. Positive bacterial cultures obtained within two days of admission and between two to thirty 
days of admissions were classified as admission or hospital acquired infections respectively.

\section{Results}

101 patients were legible for the study of which 53\% were HIV Unexposed.73\% of the total 250 cultures obtained were during admission and $44 \%$ of all the cultures obtained during admission were from blood. Gram Negative organisms are the predominantly cultured organisms in both admission and hospital acquired cultures. Escherichia Coli (Ecoli) contributes $26 \%$ of all the positive cultures on admission.

\section{Conclusion}

Gram Negative organisms remain an area of concern in both HIV Positive and HIV Negative patients with Severe Acute Malnutrition with resistant organisms more prevalent in HIV positive patients. Improving infection control practices are of vital importance in these vulnerable children to reduce the morbidity associated with hospital acquired infections.

\section{ID: 8481}

Category: Paediatric Infectious Diseases (SASPID)

Permission: Yes

Co-Author 1: Oppel, K

Co-Author 1 Institute: Stellenbosch University, Department of Paediatrics and Child Health

Co-Author 2: Holgate, $S$

Co-Author 2 Institute: Stellenbosch University, Department of Paediatrics and Child Health

Co-Author 3: Finlayson, $\mathrm{H}$

Co-Author 3 Institute: Stellenbosch University, Department of Paediatrics and Child Health

E-mail Address: kimoppel@yahoo.com

Country: South Africa

\section{ABSTRACT TITLE: A RETROSPECTIVE REVIEW OF LISTERIA MONOCYTOGENES INFECTION AT TYGERBERG HOSPITAL FROM 2006 TO 2016: IS EMPIRIC AMPICILLIN STILL INDICATED PAST THE EARLY NEONATAL PERIOD?}

\section{Introduction}

Ampicillin is added empirically to the treatment of suspected sepsis or meningitis in infants $<3$ months of age to cover Listeria Monocytogenes (LM). In view of the limited LM cases seen in other countries, the limited South African data available and the recent Ampicillin shortage at Tygerberg Hospital (TBH), we aimed to describe the positive cultures of $\mathrm{LM}$ at $\mathrm{TBH}$ to rationalize our Ampicillin usage.

\section{Methods}

This was a retrospective descriptive study of all patients with a blood or cerebral spinal fluid (CSF) culture yielding LM processed at the TBH laboratory, including Eastern Metro, Winelands and
Overberg over the 11-year period 01/01/2006 - 31/12/2016. All positive cultures of children $<13$ years of age were included in the analysis.

\section{Results}

There was a total of 26 positive cultures for $L M$ and $23 / 26(88 \%)$ $<3$ month of age, all of which were $<1$ month old. 13/23 (56.5\%) infants were managed at TBH. $6 / 13$ (46\%) presented on the day of delivery. $12 / 13(92 \%)$ were admitted to the neonatal intensive care unit (NICU) and 8/13 (62\%) died. Babies born and managed at our referral hospitals were more likely to have CSF taken ( $(90 \%$ vs $31 \%$ $(p=0.019))$, a higher platelet count $\left(239 \times 10^{\wedge} 9 / \mathrm{L}\right.$ vs $107 \times 10^{\wedge} 9 / \mathrm{L}$ $(p=0.004))$, lower CRP $(64 \mathrm{mg} / \mathrm{L}$ vs $137 \mathrm{mg} / \mathrm{L}(p=0.01))$ and a lower mortality rate $(0 \%$ vs $62 \%(p=0.002)$ than infants managed at TBH. The calculated incidence of LM at TBH was $0.04 / 1000$ live births, and 2.3/1000 NICU admissions.

\section{Conclusion}

In concordance with other countries, incidence of neonatal LM infection at TBH is low. However, infants present with severe disease and a high mortality rate. Given that no cases of neonatal LM presented $>10$ days of age, it would be safe to limit empiric Ampicillin prescription to infants $<1$ month old. This is in keeping with international guidelines from developed countries.

\section{ID: 8495}

Category: Paediatric Infectious Diseases (SASPID)

Permission: No

Co-Author 1: Rakaki , M

Co-Author 1 Institute: University of the Free State

Co-Author 2: Albertyn, J

Co-Author 2 Institute: University of the Free State

E-mail Address: rakakime@yahoo.com

Country: South Africa

\section{ABSTRACT TITLE: EXPRESSION OF ROTAVIRUS VP6 OPEN READING FRAME IN VARIOUS YEASTS AS A MORE AFFORDABLE ROTAVIRUS VACCINE CANDIDATE}

\section{Introduction}

Rotavirus infection is one of the six leading causes of death among children under five years of age. Developing countries in Sub-Saharan Africa has a high disease burden. Currently, there are two live-attenuated vaccines, Rotarix ${ }^{\mathrm{TM}}$ (GlaxoSmithKline) and RotaTeq ${ }^{\oplus}$ (Merck), recommended for global use. The efficacy of these vaccines in developing countries is much lower compared to developed countries. Due to the high cost and global demand of these vaccines, local rotavirus vaccine production is desirable. Antibodies against the immunodominant, VP6, are known to neutralise rotavirus intracellularly. Yeast as recombinant protein production vehicle, is cost-effective and scalable. A unique yeast expression system that allows for the potential recombinant protein expression in any yeast, was previously developed at the University of the Free State (UFS). 


\section{Methods}

The VP6 open reading frame (ORF) of a South African rotavirus strain was optimised for expression in Arxula adeninivorans, Pichia pastoris/angusta and Kluyveromyces lactis. The VP6 ORF was cloned into the yeast expression vector (pKM177_VP6). The UNESCO-MIRCEN yeast culture collection at UFS was targeted for recombinant protein production. Colony PCR was used to verify the integration of the VP6 ORF in each of the yeast tested. The expression of the VP6 was confirmed by western blot analysis.

\section{Results}

More than 45 yeast colonies representing five different yeast strains (Saccharomyces cerevisiae, P. pastoris, P. angusta, K. lactis and A. adeninivorans) expressing VP6 have been identified. Interestingly, results indicated that integration of the $A$. adeninivorans optimised VP6 ORF was the most effective.

\section{Conclusion}

The study provides a platform to select the best VP6-producing yeast strains. Such yeast strains will be used to scale up production for immune response studies in animals. This approach ensure that a high-yield yeast strain can be obtained to reach our long-term objective to develop a more affordable rotavirus vaccine.

\section{ID: 8513}

Category: Paediatric Infectious Diseases (SASPID)

Permission: Yes

Co-Author 1: Reddy, K

Co-Author 1 Institute: Division of Microbiology, NHLS Tygerberg and Stellenbosch University

Co-Author 2: Rabie, $\mathrm{H}$

Co-Author 2 Institute: Paediatric Infectious Diseases, Tygerberg Hospital and Stellenbosch University

Co-Author 3: Maloba, M

Co-Author 3 Institute: Division of Microbiology, NHLS Tygerberg and Stellenbosch University

Co-Author 4: Pienaar, $C$

Co-Author 4 Institute: Division of Microbiology, NHLS Tygerberg and Stellenbosch University

Co-Author 5: Gericke, $S$

Co-Author 5 Institute: Department of Paediatrics, Tygerberg Hospital and Stellenbosch University

E-mail Address: kessendri.reddy@nhls.ac.za

Country: South Africa

ABSTRACT TITLE: WILL THE REAL PATHOGEN PLEASE STAND UP? A CASE REPORT OF EXUDATIVE PHARYNGITIS CAUSED BY CORYNEBACTERIUM PSEUDODIPHTHERITICUM

\section{Introduction}

Corynebacterium pseudodiphtheriticum is a respiratory tract commensal and emerging pathogen that is closely related to
C. diphtheriae. We report a case of exudative pharyngitis from the Western Cape, ostensibly caused by C. pseudodiphtheriticum, in the aftermath of the C. diphtheriae outbreak in South Africa in 2015.

\section{Case}

A 14-month old male presented in shock with fever, features of upper airway obstruction, and an adherent, white pharyngeal membrane. Neck swelling, cranial nerve palsies and muscle weakness were absent. The patient's vaccination series was incomplete, and he was newly diagnosed as HIV-infected.

The child was resuscitated and commenced on penicillin $\mathrm{G}$, followed by cefotaxime when susceptibility was confirmed, to complete a 14-day course. Diphtheria antitoxin was not obtainable. Clinical response was protracted but steady.

Black colonies were isolated on tellurite-containing media from a throat swab, and were identified using two methods as C. pseudodiphtheriticum. Toxin testing by molecular and phenotypic methods were both negative. Antimicrobial susceptibility testing revealed susceptibility to penicillin, cefotaxime and azithromycin.

Healthcare and family contacts were screened. Two family members cultured C. pseudodiphtheriticum on throat swabs. Catch-up vaccinations and azithromycin prophylaxis were administered where appropriate.

\section{Discussion}

C. pseudodiphtheriticum has been aetiologically linked to a variety of diseases ranging from necrotising tracheitis to urinary tract infection. Respiratory tract disease is the commonest manifestation and has been reported in the presence of underlying patient risk factors, such as immunocompromise, structural lung disease, and chronic medical conditions, and following invasive respiratory procedures, including endotracheal intubation.

Only three cases of exudative pharyngitis caused by this organism have been reported, in a 32-year old male and a 4- and a 6-year old female.

C. pseudodiphtheriticum is usually susceptible to penicillin. Susceptibility to the macrolides and cefotaxime is variable; laboratory testing is advised. Management of this case required a multidisciplinary approach, including the infection control team.

This organism should be considered in the differential diagnosis of exudative pharyngitis, and further studies should attempt to define its association in this context.

\section{ID: 8523}

Category: Paediatric Infectious Diseases (SASPID)

Permission: Yes

Co-Author 1: Skosana, Z

Co-Author 1 Institute: University of Pretoria

Co-Author 2: Mbelle, $\mathrm{N}$

Co-Author 2 Institute: University of Pretoria 
Co-Author 3: von Gottberg, A

Co-Author 3 Institute: National Institute for Communicable Diseases

Co-Author 4: Olorunju, S

Co-Author 4 Institute: Medical Research Council

E-mail Address: zoteeskay@yahoo.com

Country: South Africa

\section{ABSTRACT TITLE: INVESTIGATING A REAL-TIME PCR ASSAY TO SEROTYPE PNEUMOCOCCAL CARRIAGE ISOLATES IN INFANTS AND CHILDREN PRESENTING FOR ROUTINE IMMUNISATION}

\section{Introduction}

Pneumococcal carriage is generally higher in Africa and is a prerequisite for pneumococcal disease. Pneumococcal carriage studies provide insights on the performance and impact of pneumococcal conjugate vaccines (PCV's). Real-time PCR has proven quicker and more sensitive than Quellung and conventional serotyping PCR assays. The study investigated the utility of a sequential triplex realtime PCR to serotype pneumococcal carriage isolates obtained from healthy children and infants at two primary health care centres in Gauteng between 2014 and 2016.

\section{Methods}

Three hundred and twenty-one nasopharyngeal samples were taken at specified ages and immediately processed. The epidemiological and antimicrobial susceptibility data of the samples was analysed. A 114 identified S. pneumoniaeisolates were serotyped using Quellung.Validation of the sequential triplex real-time assay for our setting was done using 77 Quellung typed invasive pneumococcal control isolates. To investigate the utility of the assay, 84 carriage isolates were subjected to molecular serotyping.

\section{Results}

Nasopharyngeal carriage of S.pneumoniae 114/309 (37\%), H.influenzae 56/309 (18\%),S. aureus 61/309 (20\%), and M.catarrhalis 5/309 (2\%) was observed; with MRSA and C. pseudodiptheriticum also isolated. The most prevalent serotypes amongst pneumococcal isolates were $11 \mathrm{~A}, 23 \mathrm{~B}$ and $15 \mathrm{~B} / \mathrm{C}$. Of 77 pneumococcal validation isolates, 74 had a serotype covered by the assay. 73 isolates yielded a positive signal, concordant with the Quellung (99\%). One isolate gave a discordant result. Two isolates had serotype identities not included in the assay with one isolate non-typeable. Of 104 pneumococcal isolates typed by Quellung, 96 isolates were assigned a serotype; four isolates could not be typed by Quellung. Of the 84 carriage isolates serotyped by the real-time assay, 48 isolates had serotypes covered by the assay. $43 / 48$ (90\%) yielded a positive result. Five of these gave discordant results.

\section{Conclusion}

The real-time assay was designed to detect 21 serotypes including those found in PCV-13, which were also detected in carriage isolates, however the assay is less useful in cases where serotype replacement has occurred.

\section{ID: 8616}

Category: Sexually Transmitted Diseases (STDSSA)

Permission: No

Author: Makua, K

Author Institute: Sefako Makgatho Health Sciences

E-mail Address: koketsomakua@gmail.com

Country: South Africa

ABSTRACT TITLE: ORAL HUMAN PAPILLOMAVIRUS INFECTION AND ASSOCIATED RISK FACTORS IN ORAL/ OROPHARYNGEAL MUCOSA OF ADULTS ATTENDING SEFAKO MAKGATHO HEALTH CARE SCIENCES ORAL HEALTH CENTRE, PRETORIA

\section{Introduction}

Evidence show the association of oral HPV infection and development of Head and Neck benign lesions and squamous cell carcinoma (HNSCC). Oro-genital sex practice is reported to be the main risk factor for oral HPV infection. However, environmental risk factors such as tobacco and alcohol consumption drive the development of HPV unassociated HNSCC. The purpose of this study is to determine the prevalence of oral HPV infection and the risk factors associated with the infection

\section{Methods}

This a cross-sectional study design aiming to enrol 250 participants. Three samples (Soft palate, right tonsillar region, floor of the mouth) were collected using Orcellex brush. The fourth sample was oral cavity wash collected using saline solution (10 $\mathrm{ml}$ saline). DNA was extracted and amplified using Abbott m2000 from oral wash and only when it was HPV DNA positive the samples from the three sites were tested. LBC slides were prepared using Thinprep T5000 system and stained with Pap stains for cytological evaluation

\section{Results}

These results are based on 150 participants. More than half (51.3\%) of the participants consume alcohol, $35.3 \%$ practice oral sex and tobacco smoking was reported in only $18.7 \%$. None of the oral wash samples had detectable HPV DNA. Sixty samples were screened for cytology. Majority (71.7\%) were negative for intraepithelial lesions or malignancy, $(18.3 \%)$ were Low grade SIL, $8.3 \%$ were ASCUS while one could be diagnosed due to poor sample (1.7\%).

\section{Conclusion}

Majority of the study participants had normal oral mucosa, and this could be attributed to the absence of HPV infection, although $35,3 \%$ reported to practice oral sex. The reason HPV DNA negativity could be the test that only detects 14 HR types. Environmental factors such as alcohol and tobacco might have played a role in the development of LSIL and ASCUS lesions. 